\title{
Identification, Synthesis and Characterization of a Major Circulating Human Metabolite of TRPV4 Antagonist GSK2798745
}

Joseph E. Pero, ${ }^{*}, \dagger$ John J. McAtee, ${ }^{\dagger}$ David J. Behm,,${ }^{\dagger}$ Jacques Briand, ${ }^{\Delta}$ Grazyna Graczyk-

Millbrandt, ${ }^{\Delta}$ Karl Erhard, ${ }^{\Delta}$ Andrew D. Roberts, ${ }^{\Delta}$ Ralph A. Rivero, ${ }^{\dagger}$ Dennis A. Holt, ${ }^{\dagger}$ and Brian

G. Lawhorn ${ }^{\dagger}$

${ }^{\dagger}$ Medicine Design, "Early Development Leaders, ${ }^{\Delta}$ Medicinal Science and Technology, GlaxoSmithKline, 1250 South Collegeville Road, Collegeville, Pa, 19426, United States

\section{SUPPORTING INFORMATION}

\section{Table of Contents}

1. Experimental procedures and compound characterization: S1-S31

2. Full characterization of 1 by 2D NMR and VCD: S31-S46

3. Comparisons of synthetic 1 to authentic GSK2708745 M1: S47-S56

\section{Experimental procedures and compound characterization}

Chemistry. All reagents and solvents were handled according to material safety data sheet of the supplier and were used as purchased without further purification.

Proton nuclear magnetic resonance ( ${ }^{1} \mathrm{H}$ NMR) and carbon-13 nuclear magnetic resonance $\left({ }^{13} \mathrm{C}\right.$ NMR) spectra were recorded with a Bruker NMR (400 or $500 \mathrm{MHz}$ ) spectrometer in the indicated solvent. FID files were processed by ACD/SpecManager (version 12.5) software. Chemical shifts are expressed in $\delta$ units (ppm) from deuterated solvent and are expressed in parts per million (ppm) units. Coupling constants $(J)$ are in units of Hertz $(\mathrm{Hz})$. Splitting patterns describe apparent multiplicities and are designated as s (singlet), $d$ (doublet), $t$ (triplet), q (quartet), dd (double doublet), dt (double triplet), m (multiplet), br (broad).

Purity of biological compounds was evaluated using Waters Acquity QDA mass detector / 4OALCMS, an Agilent 1260 or an Agilent 1100. LCMS data was generated using electrospray positive [ES+ ionization to give $\mathrm{M}+\mathrm{H}^{+}$ion, unless otherwise noted] and samples were dissolved in acetonitrile or methanol $(1 \mathrm{mg} / \mathrm{mL})$. HPLC analysis was performed by using an Sunfire C18 column $(5 \mathrm{~mm}, 3.0 \mathrm{~mm} \times 50 \mathrm{~mm})$ at $25 \pm 1{ }^{\circ} \mathrm{C}$ with an appropriate solvent gradient $(10 \%$ acetonitrile/water to $100 \%$ acetonitrile/water containing either $0.05 \%$ or $0.1 \%$ TFA), using a flow rate of $1.2 \mathrm{~mL} / \mathrm{min}$, and signal detector at 220 and $254 \mathrm{~nm}$. 
Intermediates and final products were purified by either flash column chromatography or semi-prep reverse-phase HPLC. Flash chromatography was performed on columns pre-packed with silica gel from Teledyne ISCO, unless otherwise noted. Silica gel thin layer chromatography (TLC) used for TLC were from Merck KCoA (silica gel pre-coated aluminum cards with fluorescent indicator visible at $254 \mathrm{~nm}$ ). Developed plates were visualized with a UVG-11 Compast UV lamp (254 nm).

Semi-prep HPLC was performed on an Agilent 1100. Analysis and purifications for chiral samples were performed on either an Agilent $1100 \mathrm{HPLC}$, Thar Prep 70 SFC/UV $(60 \mathrm{~mL} / \mathrm{min}$, $\mathrm{CO}_{2}$ and appropriate solvent for prep runs) or Thar Investigator SFC/UV $\left(3.0 \mathrm{~mL} / \mathrm{min}, \mathrm{CO}_{2}\right.$ and $10-50 \%$ co-solvent for analytical) using the solvent system, column and elution conditions noted in the experiment.

Evaporation of the solvents was carried out on a Büchi Rotavapor R-210 equipped with a Büchi V-850 vacuum controller and a Büchi V-700 vacuum pump.

\section{1-Methoxy-5-methylcyclohexa-1,4-diene}

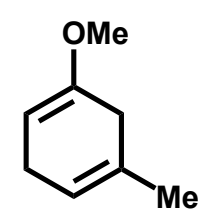

Ammonia $(400 \mathrm{~mL})$ was condensed into an evacuated, oven-dried 3-neck round bottom flask under an inert atmosphere of nitrogen at $-78{ }^{\circ} \mathrm{C}$ and equipped with a cold finger containing dry ice/acetone. To this was added 1-methoxy-3-methylbenzene $(16.6 \mathrm{~mL}, 132 \mathrm{mmol})$ in diethyl ether $(100 \mathrm{~mL})$ followed by tert-butanol $(124 \mathrm{~mL})$. Lithium wire $(4.6 \mathrm{~g}, 659 \mathrm{mmol})$ was then cautiously added in 10 equal portions. Following the addition, the reaction mixture was warmed to $-33^{\circ} \mathrm{C}$, after which it turned dark blue in color. The reaction contents were stirred at $-33^{\circ} \mathrm{C}$ for $3 \mathrm{~h}$, after which time ammonium chloride $(50 \mathrm{~g}, 935 \mathrm{mmol}$ ) was added in 5 equal portions. The reaction mixture was subsequently removed from the $-33{ }^{\circ} \mathrm{C}$ bath, the cold finger was removed and the reaction contents (open to the atmosphere) were allowed to warm to RT and stirred for an additional $14 \mathrm{~h}$. The resulting organic material was dissolved in $500 \mathrm{~mL}$ of pentane and washed with water $(3 \times 100 \mathrm{~mL})$. The organics were dried over anhydrous $\mathrm{Na}_{2} \mathrm{SO}_{4}$, filtered, and concentrated in vacuo (bath temperature $=25^{\circ} \mathrm{C}, 50$ mbar) to give a clear, pale yellow oil (29.6 g). ${ }^{1} \mathrm{H}$ NMR analysis was consistent with the title compound as a solution in tert-butanol, which was carried forward without further manipulation. ${ }^{1} \mathrm{H}$ NMR $\left(400 \mathrm{MHz}, \mathrm{CDCl}_{3}\right) \delta \mathrm{ppm} 1.30-1.35$ 
$(\mathrm{m}, 3 \mathrm{H}) 2.55-2.65(\mathrm{~m}, 2 \mathrm{H}) 2.73-2.87(\mathrm{~m}, 2 \mathrm{H}) 3.58(\mathrm{~s}, 3 \mathrm{H}) 4.58$ - $4.70(\mathrm{~m}, 1 \mathrm{H}) 5.35$ - $5.48(\mathrm{~m}$, $1 \mathrm{H})$.

\section{3-Methylcyclohex-3-en-1-one}

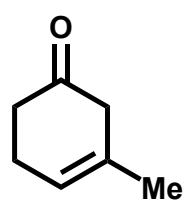

To a solution of 1-methoxy-5-methylcyclohexa-1,4-diene $(1.28 \mathrm{~g}, 10.3 \mathrm{mmol})$ in methanol $(16 \mathrm{~mL})$ and water $(5 \mathrm{~mL})$ was added oxalic acid $(0.05 \mathrm{~g}, 0.51 \mathrm{mmol})$ in one portion. After 90 min at RT, the reaction contents were poured into $16 \mathrm{~mL}$ water and extracted with $\mathrm{DCM}(3 \times 12 \mathrm{~mL})$. The combined organics were dried over anhydrous $\mathrm{Na}_{2} \mathrm{SO}_{4}$, filtered and concentrated in vacuo (bath temp $=25^{\circ} \mathrm{C}$, pressure $=50 \mathrm{mbar}$ ) to give the title compound as a clear, yellow oil (573 mg, 5.2 mmol, 51\%). ${ }^{1} \mathrm{H}$ NMR (400 MHz, $\left.\mathrm{CDCl}_{3}\right) \delta$ ppm 1.71 - $1.77(\mathrm{~m}, 3 \mathrm{H}) 2.39$ - $2.49(\mathrm{~m}, 4 \mathrm{H}) 2.80$ (s, 2 H) 5.61 (br. s., 1 H).

\section{1-Methyl-7-oxabicyclo[4.1.0]heptan-3-one}

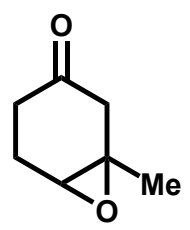

To a slurry of $m$-CPBA (23.42 g, $136 \mathrm{mmol}$ ) in dichloromethane (DCM) (348 $\mathrm{mL})$ was added a solution of 3-methylcyclohex-3-en-1-one (11.5 g, $104 \mathrm{mmol})$ in dichloromethane (DCM) (70 mL) via cannula. After $2.5 \mathrm{~h}$ at $0{ }^{\circ} \mathrm{C}$, the reaction was quenched with $200 \mathrm{~mL}$ saturated aqueous $\mathrm{Na}_{2} \mathrm{~S}_{2} \mathrm{O}_{3}$ and allowed to stir for $5 \mathrm{~min}$. The layers were separated and the aqueous layer was extracted with DCM $(2 \times 150 \mathrm{~mL})$. The combined organics were washed with saturated aqueous $\mathrm{NaHCO}_{3}(5 \times 100 \mathrm{~mL})$, dried over anhydrous $\mathrm{Na}_{2} \mathrm{SO}_{4}$, filtered and concentrated to give the title compound as a clear, orange oil $\left(11.5 \mathrm{~g}, 91.9 \mathrm{mmol}, 87 \%\right.$ yield). ${ }^{1} \mathrm{H} \mathrm{NMR}\left(400 \mathrm{MHz}^{\left.-\mathrm{CDCl}_{3}\right) \delta}\right.$ ppm $1.40(\mathrm{~s}, 3 \mathrm{H}) 2.15$ - $2.25(\mathrm{~m}, 2 \mathrm{H}) 2.34$ - $2.46(\mathrm{~m}, 2 \mathrm{H}) 2.56-2.65(\mathrm{~m}, 1 \mathrm{H}) 2.75$ - 2.87 (m, 1 H) $3.25(\mathrm{~d}, J=2.26 \mathrm{~Hz}, 1 \mathrm{H})$. 


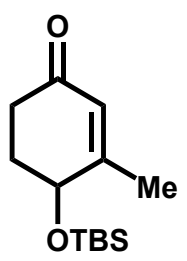

To a solution of 1-methyl-7-oxabicyclo[4.1.0]heptan-3-one $(11.5 \mathrm{~g}, 91 \mathrm{mmol})$ in dichloromethane (DCM) (304 mL) at RT was added sequentially diisopropylethylamine $(32.6 \mathrm{ml}, 187 \mathrm{mmol})$, tertbutyldimethylsilyl chloride $(15.1 \mathrm{~g}, 100 \mathrm{mmol})$ and DMAP $(2.2 \mathrm{~g}, 18.2 \mathrm{mmol})$. After $18 \mathrm{~h}$, the reaction contents were partitioned with $200 \mathrm{~mL}$ of EtOAc and $100 \mathrm{~mL}$ of saturated aqueous $\mathrm{NaHCO}_{3}$ and the layers were separated. The aqueous layer was back-extracted with EtOAc $(3 \mathrm{x}$ $30 \mathrm{~mL}$ ). The combined organics were dried over anhydrous $\mathrm{Na}_{2} \mathrm{SO}_{4}$, filtered and concentrated in vacuo to give an orange oil. The crude product was purified by silica gel chromatography $(750 \mathrm{~g}$ column $100 \% \mathrm{DCM})$ to give the title compound as a clear orange oil $(11.4 \mathrm{~g}, 47.4 \mathrm{mmol}, 52 \%$ yield). ${ }^{1} \mathrm{H}$ NMR $\left(400 \mathrm{MHz}, \mathrm{CDCl}_{3}\right) \delta \mathrm{ppm} 0.16$ (d, J=3.51 Hz, $\left.6 \mathrm{H}\right) 0.95$ (s, $\left.9 \mathrm{H}\right) 1.95$ - 2.06 (m, 4 H) 2.18 (br. s., 1 H) 2.34 (s, 1 H) 2.56 (s, 1 H) 4.37 (d, J=3.01 Hz, 1 H) 5.85 (s, 1 H).

\section{(3S,4R)-4-((tert-Butyldimethylsilyl)oxy)-3-methyl-3-(nitromethyl)cyclohexan-1-one (3)} (3S,4S)-4-((tert-Butyldimethylsilyl)oxy)-3-methyl-3-(nitromethyl)cyclohexan-1-one (18)
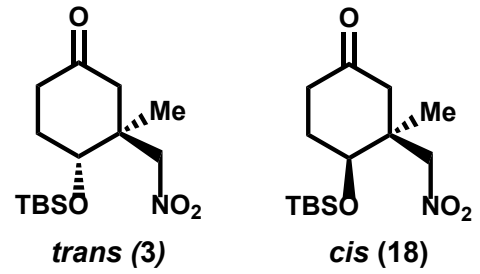

To a solution of 4-((tert-butyldimethylsilyl)oxy)-3-methylcyclohex-2-en-1-one (11.4 g, $47.4 \mathrm{mmol})$ in dichloromethane $(\mathrm{DCM})(158 \mathrm{~mL})$ was added $(R)$-proline $(1.1 \mathrm{~g}, 9.5 \mathrm{mmol}), 2$-methylpiperidine $(19.0 \mathrm{~mL}, 161 \mathrm{mmol})$ and nitromethane $(10.2 \mathrm{~mL}, 190 \mathrm{mmol})$. The reaction mixture was warmed to $40{ }^{\circ} \mathrm{C}$ and stirred for $17 \mathrm{~h}$, after which time the reaction was quenched with $200 \mathrm{~mL}$ of water and the layers were separated. The aqueous layer was extracted with DCM $(3 \times 50 \mathrm{~mL})$ and the combined organics were dried over anhydrous $\mathrm{Na}_{2} \mathrm{SO}_{4}$, filtered and concentrated in vacuo to give a brown oil. Purification by silica gel chromatography (750 g RediSep column, 100\% DCM) afforded a 1:1 mixture of trans- and cis-diastereomers $(12.3 \mathrm{~g})$. The diastereomers were separated via preparative chiral HPLC (Chiralpak IC, 5 н, 30 × 250 mm, 95:5 Heptane:EtOH, 50 
$\mathrm{mL} / \mathrm{min}$ flow rate) to give $3(3.0 \mathrm{~g}, 21 \%$ yield, $74 \%$ ee $)$ and $18(4.6 \mathrm{~g}, 32 \%$ yield, $72 \%$ ee $)$ as colorless oils.

3: LC-MS $\mathrm{C}_{14} \mathrm{H}_{27} \mathrm{NO}_{4} \mathrm{Si}[\mathrm{M}+\mathrm{H}]$ calculated: 302.2, observed: 302.3, 1.32 min (ret. time); ${ }^{1} \mathrm{H} \mathrm{NMR}$ $\left(400 \mathrm{MHz}, \mathrm{CDCl}_{3}\right) \delta$ ppm $0.16(\mathrm{~s}, 6 \mathrm{H}) 0.95(\mathrm{~s}, 9 \mathrm{H}) 1.09(\mathrm{~s}, 3 \mathrm{H}) 1.86-2.01(\mathrm{~m}, 1 \mathrm{H}) 2.07$ - 2.17 $(\mathrm{m}, 1 \mathrm{H}) 2.33-2.41(\mathrm{~m}, 1 \mathrm{H}) 2.43-2.46(\mathrm{~m}, 2 \mathrm{H}) 2.47-2.54(\mathrm{~m}, 1 \mathrm{H}) 4.05-4.11(\mathrm{~m}, 1 \mathrm{H}) 4.23$ $4.29(\mathrm{~m}, 1 \mathrm{H}) 4.44-4.50(\mathrm{~m}, 1 \mathrm{H})$.

Enantiomeric purity assessed by chiral analytical HPLC (Chiralpak IC, $5 \mu, 4.6$ x 150 mm, 97:3 Heptane:EtOH, $1 \mathrm{~mL} / \mathrm{min}$ flow rate) using authentic (3R,4S)-4-((tert-butyldimethylsilyl)oxy)-3methyl-3-(nitromethyl)cyclohexan-1-one acquired from an analogous reaction involving $(S)$ proline as organocatalyst: Retention time $(3 R, 4 S)=11.2 \mathrm{~min}$, retention time $(3 S, 4 R)=12.0 \mathrm{~min}$. $\%$ ee determination of $6(3 S, 4 R)=74 \%$

18: LC-MS $\mathrm{C}_{14} \mathrm{H}_{28} \mathrm{NO}_{4} \mathrm{Si}[\mathrm{M}+\mathrm{H}]$ calculated: 302.2 , observed: $302.3,1.28 \mathrm{~min}$ (ret. time); ${ }^{1} \mathrm{H} \mathrm{NMR}$ $\left(400 \mathrm{MHz}, \mathrm{CDCl}_{3}\right) \delta$ ppm $0.19(\mathrm{~s}, 6 \mathrm{H}) 0.97(\mathrm{~s}, 9 \mathrm{H}) 1.07(\mathrm{~s}, 3 \mathrm{H}) 2.00-2.05(\mathrm{~m}, 2 \mathrm{H}) 2.06-2.12$ $(\mathrm{m}, 1 \mathrm{H}) 2.24-2.33(\mathrm{~m}, 1 \mathrm{H}) 2.61-2.73(\mathrm{~m}, 2 \mathrm{H}) 4.04(\mathrm{br} \mathrm{s}, 1 \mathrm{H}) 4.20(\mathrm{~d}, \mathrm{~J}=11.04 \mathrm{~Hz}, 1 \mathrm{H}) 4.67$ (d, J=11.04 Hz, $1 \mathrm{H}$ ).

Enantiomeric purity assessed by chiral analytical HPLC (Chiralpak IC, $5 \mu, 4.6$ x 150 mm, 97:3 Heptane:EtOH, $1 \mathrm{~mL} / \mathrm{min}$ flow rate) using authentic (3R,4R)-4-((tert-butyldimethylsilyl)oxy)-3methyl-3-(nitromethyl)cyclohexan-1-one acquired from an analogous reaction involving $(S)$ proline as organocatalyst: Retention time $(3 R, 4 R)=6.4 \mathrm{~min}$, retention time $(3 S, 4 S)=7.2 \mathrm{~min}$. \%ee determination of $18(3 S, 4 S)=72 \%$.

\section{tert-Butyldimethyl(((7S,8R)-7-methyl-7-(nitromethyl)-1,4-dioxaspiro[4.5]decan-8- yl)oxy)silane}

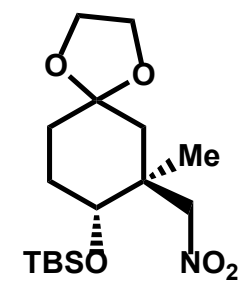

To a solution of (3S,4R)-4-((tert-butyldimethylsilyl)oxy)-3-methyl-3-(nitromethyl)cyclohexan-1-one $(1.5 \mathrm{~g}, 5.0 \mathrm{mmol})$ in dichloromethane $(\mathrm{DCM})(1.3 \mathrm{~mL})$ was added ethylene glycol $(0.42 \mathrm{ml}, 7.46$ $\mathrm{mmol})$ and trimethylorthoformate $(0.83 \mathrm{ml}, 7.5 \mathrm{mmol})$. The resulting reaction mixture was stirred at RT for $5 \mathrm{~min}$, then cooled to $0{ }^{\circ} \mathrm{C}$ in an ice bath. To this mixture was added methanesulfonic acid $(0.048 \mathrm{ml}, 0.746 \mathrm{mmol})$ dropwise. The reaction mixture was removed from the bath and allowed to warm to RT. After $90 \mathrm{~min}$, the reaction was cooled back to $0{ }^{\circ} \mathrm{C}$, quenched with $10 \mathrm{~mL}$ 
water and partitioned with $10 \mathrm{~mL}$ DCM. The layers were separated and the aqueous layer was extracted with DCM $(3 \times 5 \mathrm{~mL})$. The combined organic layers were dried over anhydrous $\mathrm{Na}_{2} \mathrm{SO}_{4}$, filtered and concentrated to give a clear, yellow oil. The crude material was purified by silica gel chromatography (40 g RediSep Gold column, 0-20\% Acetone:Hexanes) to give the title compound as a clear, colorless oil (1.3 g, $3.6 \mathrm{mmol}, 73 \%$ yield). LC-MS $\mathrm{C}_{16} \mathrm{H}_{32} \mathrm{NO}_{5} \mathrm{Si}[\mathrm{M}+\mathrm{H}]$ calculated: 346.2 , observed: $346.3,1.46 \mathrm{~min}$ (ret. time); ${ }^{1} \mathrm{H} \mathrm{NMR}\left(400 \mathrm{MHz}, \mathrm{CDCl}_{3}\right) \delta \mathrm{ppm} 0.10$ (d, J=5.52 Hz, 6 H) $0.93(\mathrm{~s}, 9 \mathrm{H}) 1.11(\mathrm{~s}, 3 \mathrm{H}) 1.63(\mathrm{~d}, \mathrm{~J}=14.31 \mathrm{~Hz}, 2 \mathrm{H}) 1.76-1.96(\mathrm{~m}, 4 \mathrm{H}) 3.69$ - $3.76(\mathrm{~m}, 1 \mathrm{H}) 3.95$ (br. s., $4 \mathrm{H}) 4.45$ - $4.50(\mathrm{~m}, 1 \mathrm{H}) 4.51$ - $4.57(\mathrm{~m}, 1 \mathrm{H})$.

((7S,8R)-8-((tert-Butyldimethylsilyl)oxy)-7-methyl-1,4-dioxaspiro[4.5]decan-7-

\section{yl)methanamine (4)}

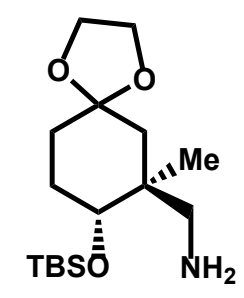

A solution of tert-butyldimethyl(((7S,8R)-7-methyl-7-(nitromethyl)-1,4-dioxaspiro[4.5]decan-8$\mathrm{yl})$ oxy)silane $(1.3 \mathrm{~g}, 3.8 \mathrm{mmol})$ in methanol $(18.1 \mathrm{~mL})$ was continuously run through an $\mathrm{H}$-Cube flow reactor equipped with a $10 \% \mathrm{Pd} / \mathrm{C}$ cartridge under the following conditions: $70^{\circ} \mathrm{C}, 10$ bar, 1 $\mathrm{mL} /$ min pump rate. After $2 \mathrm{~h}$, LCMS analysis confirmed consumption of starting material and the $\mathrm{H}$-Cube flow reactor was flushed with ethanol $(1 \mathrm{~mL} / \mathrm{min}$ for $10 \mathrm{~min})$. The reaction mixture was concentrated in vacuo to provide the title compound as a clear, colorless oil $(1.1 \mathrm{~g}, 3.5 \mathrm{mmol}$, 92\% yield). LC-MS $\mathrm{C}_{16} \mathrm{H}_{34} \mathrm{NO}_{3} \mathrm{Si}[\mathrm{M}+\mathrm{H}]$ calculated: 316.2, observed: 316.0, $0.92 \mathrm{~min}$ (ret. time); ${ }^{1} \mathrm{H} \mathrm{NMR}\left(400 \mathrm{MHz}, \mathrm{CDCl}_{3}\right) \delta \mathrm{ppm} 0.09(\mathrm{~s}, 6 \mathrm{H}) 0.91(\mathrm{~s}, 9 \mathrm{H}) 1.17(\mathrm{~s}, 3 \mathrm{H}) 1.68-1.75(\mathrm{~m}, 2 \mathrm{H})$ 1.76 - $1.83(\mathrm{~m}, 2 \mathrm{H}) 1.86-1.92(\mathrm{~m}, 2 \mathrm{H}) 2.84-2.92(\mathrm{~m}, 1 \mathrm{H}) 3.12-3.20(\mathrm{~m}, 1 \mathrm{H}) 3.60$ - $3.66(\mathrm{~m}$, $1 \mathrm{H}) 3.82$ - $3.89(\mathrm{~m}, 1 \mathrm{H}) 3.90$ - $3.94(\mathrm{~m}, 1 \mathrm{H}) 3.99$ - $4.06(\mathrm{~m}, 1 \mathrm{H}) 4.10-4.21(\mathrm{~m}, 1 \mathrm{H}) 8.22-8.44$ (m, $2 \mathrm{H})$. 
3-((((7S,8R)-8-((tert-Butyldimethylsilyl)oxy)-7-methyl-1,4-dioxaspiro[4.5]decan-7yl)methyl)amino)-4-nitrobenzonitrile (5)

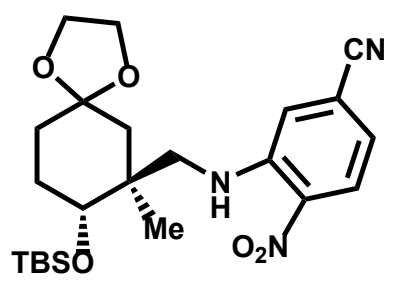

To a suspension ((7S,8R)-8-((tert-butyldimethylsilyl)oxy)-7-methyl-1,4-dioxaspiro[4.5]decan-7$\mathrm{yl})$ methanamine $(1.1 \mathrm{~g}, 3.5 \mathrm{mmol})$ in acetonitrile $(11.5 \mathrm{~mL})$ was added 3-fluoro-4-nitrobenzonitrile $(0.574 \mathrm{~g}, 3.45 \mathrm{mmol})$ and $\mathrm{K}_{2} \mathrm{CO}_{3}(1.4 \mathrm{~g}, 10.4 \mathrm{mmol})$ sequentially. The resulting reaction mixture was warmed to $40{ }^{\circ} \mathrm{C}$ bath. After $90 \mathrm{~min}$, the reaction contents were partitioned with $100 \mathrm{~mL}$ of EtOAc and $50 \mathrm{~mL}$ of saturated aqueous $\mathrm{NaHCO}_{3}$, and the layers were separated. The aqueous layer was extracted with EtOAc $(3 \times 30 \mathrm{~mL})$. The combined organics were dried over anhydrous $\mathrm{Na}_{2} \mathrm{SO}_{4}$, filtered and concentrated in vacuo to give an orange oil that rapidly solidified upon standing at RT. The crude material was purified by silica gel chromatography $(80 \mathrm{~g}$ RediSep Gold column, $0-20 \%$ EtOAc:Hexanes) to give the title compound as an orange solid $(1.4 \mathrm{~g}, 3.1 \mathrm{mmol}$, 89\%). LC-MS $\mathrm{C}_{23} \mathrm{H}_{36} \mathrm{~N}_{3} \mathrm{O}_{5} \mathrm{Si}[\mathrm{M}+\mathrm{H}]$ calculated: 462.2, observed: 462.5, 1.62 min (ret. time); ${ }^{1} \mathrm{H}$ NMR (400 MHz, CDCl $\left.)_{3}\right) \delta \mathrm{ppm} 0.11(\mathrm{~d}, \mathrm{~J}=6.78 \mathrm{~Hz}, 6 \mathrm{H}) 0.94(\mathrm{~s}, 9 \mathrm{H}) 1.10(\mathrm{~s}, 3 \mathrm{H}) 1.72$ - $1.97(\mathrm{~m}$, $6 \mathrm{H}) 3.28(\mathrm{~d}, \mathrm{~J}=6.27 \mathrm{~Hz}, 1 \mathrm{H}) 3.39$ (br. s., $1 \mathrm{H}) 3.64$ (br. s., $1 \mathrm{H}) 3.90-4.00(\mathrm{~m}, 4 \mathrm{H}) 6.84$ (d, $\mathrm{J}=8.78 \mathrm{~Hz}, 1 \mathrm{H}) 7.25(\mathrm{~s}, 1 \mathrm{H}) 8.27(\mathrm{~d}, \mathrm{~J}=8.53 \mathrm{~Hz}, 1 \mathrm{H})$.

\section{4-Amino-3-((((7S,8R)-8-((tert-butyldimethylsilyl)oxy)-7-methyl-1,4-dioxaspiro[4.5]decan-7- yl)methyl)amino)benzonitrile}

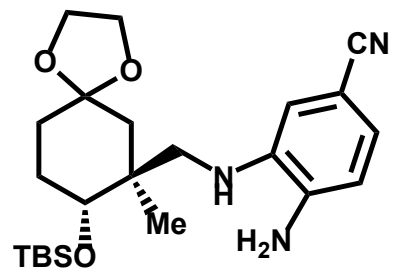

To $10 \% \mathrm{Pd} / \mathrm{C}(627 \mathrm{mg}, 0.589 \mathrm{mmol})$ under an atmosphere of $\mathrm{N}_{2}$ was added a solution of 3((((7S,8R)-8-((tert-butyldimethylsilyl)oxy)-7-methyl-1,4-dioxaspiro[4.5]decan-7-yl)methyl)amino)4-nitrobenzonitrile $(1.4 \mathrm{~g}, 3.0 \mathrm{mmol})$ in ethyl acetate $(6.6 \mathrm{~mL})$. To this mixture was added methanol (13.1 $\mathrm{mL}$ ) and the reaction vessel was evacuated and back-filled with $\mathrm{H}_{2}$ from a balloon. The evacuation/back-fill process was repeated an additional two times. The reaction was stirred at RT for $90 \mathrm{~min}$, the reaction vessel was evacuated and back-filled with nitrogen. The reaction contents were filtered through Celite, washed with $25 \mathrm{~mL}$ EtOAc and concentrated in vacuo to give a clear, 
brown oil. The crude material was purified by silica gel chromatography (40 g RediSep Gold column, 0-40\% EtOAc:Hexanes) to give the title compound as a pale yellow oil $(1.3 \mathrm{~g}, 2.9 \mathrm{mmol}$, $100 \%$ yield). LC-MS $\mathrm{C}_{23} \mathrm{H}_{37} \mathrm{~N}_{3} \mathrm{O}_{3} \mathrm{Si}[\mathrm{M}+\mathrm{H}]$ calculated: 432.3, observed: 432.5, 1.40 min (ret. time); ${ }^{1} \mathrm{H}$ NMR (400 MHz, DMSO-d6) $\delta$ ppm -0.04 - 0.11 (m, 6 H) 0.87 (s, 9 H) 1.01 (s, 3 H) 1.49 - 1.80 $(\mathrm{m}, 6 \mathrm{H}) 2.94(\mathrm{~d}, \mathrm{~J}=5.02 \mathrm{~Hz}, 2 \mathrm{H}) 3.31(\mathrm{~s}, 1 \mathrm{H}) 3.81$ (d, J=5.77 Hz, 2 H) 3.87 (d, J=5.77 Hz, 2 H) 4.20 (br. s., 1 H) 5.72 (br. s., 2 H) 6.58 (d, J=8.03 Hz, 1 H) 6.62 (s, 1 H) 6.85 (d, J=7.78 Hz, 1 H).

\section{1-(((7S,8R)-8-((tert-Butyldimethylsilyl)oxy)-7-methyl-1,4-dioxaspiro[4.5]decan-7-}

\section{yl)methyl)-1H-benzo[d]imidazole-6-carbonitrile (6)}

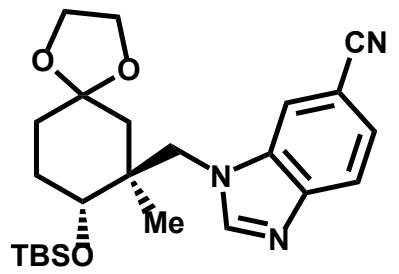

4-Amino-3-((((7S,8R)-8-((tert-butyldimethylsilyl)oxy)-7-methyl-1,4-dioxaspiro[4.5]decan-7$\mathrm{yl}$ )methyl)amino)benzonitrile $(501 \mathrm{mg}, 1.2 \mathrm{mmol}$ ) was added to trimethyl orthoformate $(3.8 \mathrm{~mL}$, $34.8 \mathrm{mmol})$ followed by formic acid $(45 \mu \mathrm{L}, 1.2 \mathrm{mmol})$. The resulting mixture was allowed to stir at RT. An additional $45 \mu \mathrm{L}$ of formic acid was added at $\mathrm{t}=90 \mathrm{~min}, 120 \mathrm{~min}$, and $180 \mathrm{~min}$ following the initiation of the reaction, after which the mixture was allowed to stir for an additional $16 \mathrm{~h}$. The reaction contents were partitioned with $50 \mathrm{~mL}$ of EtOAc and $30 \mathrm{~mL}$ of saturated aqueous $\mathrm{NaHCO}_{3}$ and the layers were separated. The aqueous layer was back-extracted with EtOAc ( $3 \times 15 \mathrm{~mL})$. The combined organics were dried over anhydrous $\mathrm{Na}_{2} \mathrm{SO}_{4}$, filtered and concentrated in vacuo to give a pale yellow oil. The crude material was purified by silica gel chromatography $(40 \mathrm{~g}$ RediSep Gold column, 0-70\% EtOAc:Hexanes) to give the title compound as a white solid (534 mg, $1.2 \mathrm{mmol}, 100 \%)$. LC-MS $\mathrm{C}_{24} \mathrm{H}_{35} \mathrm{~N}_{3} \mathrm{O}_{3} \mathrm{Si}[\mathrm{M}+\mathrm{H}$ ] calculated: 442.2, observed: 442.6, $1.33 \mathrm{~min}$ (ret. time); ${ }^{1} \mathrm{H}$ NMR (400 MHz, DMSO-d $\left.d_{6}\right) \delta$ ppm $0.12(\mathrm{~s}, 6 \mathrm{H}) 0.87(\mathrm{~s}, 3 \mathrm{H}) 0.93(\mathrm{~s}, 9 \mathrm{H}) 1.36$ (d, $\mathrm{J}=13.55 \mathrm{~Hz}, 1 \mathrm{H}) 1.50$ - $1.62(\mathrm{~m}, 2 \mathrm{H}) 1.71(\mathrm{~d}, \mathrm{~J}=8.28 \mathrm{~Hz}, 2 \mathrm{H}) 1.79-1.90(\mathrm{~m}, 1 \mathrm{H}) 3.75$ - $3.90(\mathrm{~m}$, $5 \mathrm{H}) 4.18$ - $4.26(\mathrm{~m}, 1 \mathrm{H}) 4.32-4.40(\mathrm{~m}, 1 \mathrm{H}) 7.60(\mathrm{~d}, \mathrm{~J}=8.28 \mathrm{~Hz}, 1 \mathrm{H}) 7.84(\mathrm{~d}, \mathrm{~J}=8.53 \mathrm{~Hz}, 1 \mathrm{H})$ $8.33(\mathrm{~s}, 1 \mathrm{H}) 8.40(\mathrm{~s}, 1 \mathrm{H})$. 
1-(((1S,2R)-2-((tert-Butyldimethylsilyl)oxy)-1-methyl-5-oxocyclohexyl)methyl)-1Hbenzo[d]imidazole-6-carbonitrile (7)

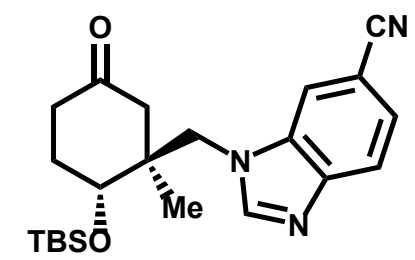

Formic acid (3.3 mL, $86 \mathrm{mmol})$ was added to 1-(((7S,8R)-8-((tert-butyldimethylsilyl)oxy)-7-methyl1,4-dioxaspiro[4.5]decan-7-yl)methyl)-1H-benzo[d]imidazole-6-carbonitrile $\quad(377.8 \mathrm{mg}, \quad 0.855$ $\mathrm{mmol}$ ) and the resulting mixture was allowed to stir at RT. After $70 \mathrm{~min}$, the reaction contents were partitioned with $100 \mathrm{~mL}$ of EtOAc and $100 \mathrm{~mL}$ of saturated aqueous $\mathrm{NaHCO}_{3}$ and the layers were separated. The organics were washed with saturated aqueous $\mathrm{NaHCO}_{3}(1 \times 50 \mathrm{~mL})$ and the layers were separated. The combined aqueous layer was back-extracted with EtOAc ( $3 \times 20$ $\mathrm{mL}$ ). The combined organics were dried over anhydrous $\mathrm{Na}_{2} \mathrm{SO}_{4}$, filtered and concentrated in vacuo to give a pale yellow oil which solidified to a white solid upon placing on a high-vacuum line (433 mg). ${ }^{1} \mathrm{H}$ NMR analysis consistent with the title compound plus minor impurities. The impure material was carried forward without further purification. LC-MS $\mathrm{C}_{22} \mathrm{H}_{31} \mathrm{~N}_{3} \mathrm{O}_{2} \mathrm{Si}[\mathrm{M}+\mathrm{H}]$, calculated: 398.2, observed: 398.5, $1.22 \mathrm{~min}$ (ret. time); ${ }^{1} \mathrm{H}$ NMR (400 MHz, DMSO-d $\left.d_{6}\right) \delta$ ppm 0.19 (d, J=7.03 $\mathrm{Hz}, 6 \mathrm{H}) 0.85(\mathrm{~s}, 3 \mathrm{H}) 0.96(\mathrm{~s}, 9 \mathrm{H}) 1.88(\mathrm{dd}, \mathrm{J}=13.55,1.76 \mathrm{~Hz}, 2 \mathrm{H}) 2.10-2.19(\mathrm{~m}, 1 \mathrm{H}) 2.36(\mathrm{~d}$, $\mathrm{J}=13.80 \mathrm{~Hz}, 3 \mathrm{H}) 4.08-4.16(\mathrm{~m}, 1 \mathrm{H}) 4.19-4.25(\mathrm{~m}, 1 \mathrm{H}) 4.31-4.37(\mathrm{~m}, 1 \mathrm{H}) 7.61$ (dd, J=8.41, $1.38 \mathrm{~Hz}, 1 \mathrm{H}) 7.85(\mathrm{~d}, \mathrm{~J}=8.53 \mathrm{~Hz}, 1 \mathrm{H}) 8.31(\mathrm{~d}, \mathrm{~J}=0.75 \mathrm{~Hz}, 1 \mathrm{H}) 8.38(\mathrm{~s}, 1 \mathrm{H})$.

1-(((3S,5S,6R)-6-((tert-Butyldimethylsilyl)oxy)-5-methyl-1-oxaspiro[2.5]octan-5-yl)methyl)1H-benzo[d]imidazole-6-carbonitrile (8)

1-(((3R,5S,6R)-6-((tert-Butyldimethylsilyl)oxy)-5-methyl-1-oxaspiro[2.5]octan-5-yl)methyl)1H-benzo[d]imidazole-6-carbonitrile (18)

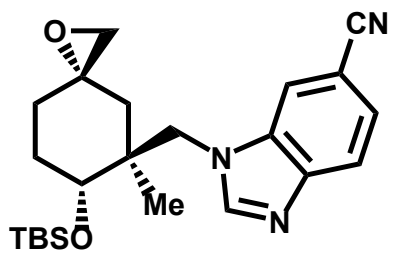

8

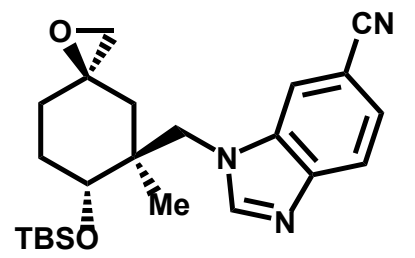

18

$\mathrm{NaH}$ (174 mg, $4.35 \mathrm{mmol}, 60 \%$ dispersion in oil) was added to a solution of trimethylsulfoxonium iodide (956 mg, $4.35 \mathrm{mmol}$ ) in dimethyl sulfoxide (DMSO) (33 mL) and the resulting mixture was stirred at RT for $30 \mathrm{~min}$. To this mixture was added a solution of impure 1-(((1S,2R)-2-((tertbutyldimethylsilyl)oxy)-1-methyl-5-oxocyclohexyl)methyl)-1H-benzo[d]imidazole-6-carbonitrile 
(432 $\mathrm{mg}$ ) in dimethyl sulfoxide (DMSO) $(11 \mathrm{~mL})$ dropwise. After $8 \mathrm{~min}$, the reaction mixture was cooled to $0{ }^{\circ} \mathrm{C}$, quenched slowly with $30 \mathrm{~mL}$ water and partitioned with $150 \mathrm{~mL}$ of EtOAc. The layers were separated and the aqueous layer was back-extracted with EtOAc (1 x $40 \mathrm{~mL})$. The combined organics were washed with water $(4 \times 50 \mathrm{~mL})$ and brine $(1 \times 50 \mathrm{~mL})$. The organics were dried over anhydrous $\mathrm{Na}_{2} \mathrm{SO}_{4}$, filtered and concentrated in vacuo to give a pale yellow oil. The crude product was purified by silica gel chromatography (80 g RediSep Gold column, 0-50\% EtOAc:Hexanes) to give $8(80.3 \mathrm{mg}, 0.185 \mathrm{mmol}, 17 \%$ yield over 2 steps) and 18 (365.5 mg, $0.879 \mathrm{mmol}, 81 \%$ yield over 2 steps). Both diastereomers were isolated as white solids.

8: LC-MS $\mathrm{C}_{23} \mathrm{H}_{33} \mathrm{~N}_{3} \mathrm{O}_{2} \mathrm{Si}[\mathrm{M}+\mathrm{H}]$ calculated: 412.2, observed: 412.5, 1.27 min (ret. time); ${ }^{1} \mathrm{H} \mathrm{NMR}$ (400 MHz, DMSO-d $)_{6} \delta$ ppm $0.16(\mathrm{~d}, \mathrm{~J}=4.52 \mathrm{~Hz}, 6 \mathrm{H}) 0.97(\mathrm{~s}, 12 \mathrm{H}) 1.22$ - $1.30(\mathrm{~m}, 2 \mathrm{H}) 1.68$ $1.79(\mathrm{~m}, 1 \mathrm{H}) 1.81-1.89(\mathrm{~m}, 2 \mathrm{H}) 1.93-2.01(\mathrm{~m}, 1 \mathrm{H}) 2.42(\mathrm{~d}, \mathrm{~J}=7.78 \mathrm{~Hz}, 2 \mathrm{H}) 3.80$ - $3.88(\mathrm{~m}, 1$ H) $4.28(\mathrm{~s}, 2 \mathrm{H}) 7.54-7.63(\mathrm{~m}, 1 \mathrm{H}) 7.78-7.87(\mathrm{~m}, 1 \mathrm{H}) 8.26(\mathrm{~s}, 1 \mathrm{H}) 8.40(\mathrm{~s}, 1 \mathrm{H})$.

18: LC-MS $\mathrm{C}_{23} \mathrm{H}_{33} \mathrm{~N}_{3} \mathrm{O}_{2} \mathrm{Si}[\mathrm{M}+\mathrm{H}]$ calculated: 412.2, observed: 412.5, 1.30 min (ret. time); ${ }^{1} \mathrm{H}$ NMR $\left(500 \mathrm{MHz}, \mathrm{DMSO}_{-} \mathrm{d}_{6}\right) \delta \mathrm{ppm} 8.42$ (s, 1H), 8.36 (s, 1H), 7.83 (d, J=8.4 Hz, 1H), 7.59 (dd, J=8.4, $1.3 \mathrm{~Hz}, 1 \mathrm{H}), 4.38$ (d, J=14.3 Hz, 1H), 4.24 (d, J=14.3 Hz, 1H), 3.88 (dd, J=7.7, 3.3 Hz, 1H), 2.53$2.61(\mathrm{~m}, 2 \mathrm{H}), 1.92-1.97(\mathrm{~m}, 1 \mathrm{H}), 1.68-1.75(\mathrm{~m}, 1 \mathrm{H}), 1.62-1.68(\mathrm{~m}, 1 \mathrm{H}), 1.57(\mathrm{br} \mathrm{d}, J=14.0 \mathrm{~Hz}, 1 \mathrm{H})$, 1.49-1.55 (m, 2H), 1.17 (br d, J=13.2 Hz, 1H), 0.94 (s, 9H), 0.85 (s, 3H), 0.15 (d, J=4.1 Hz, 6H).

Structure elucidation of 18 :

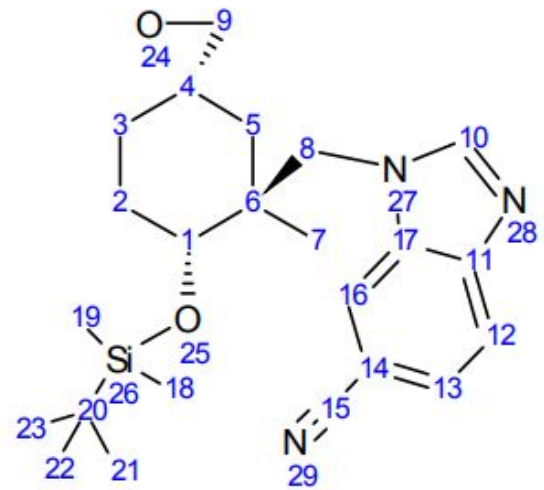




\begin{tabular}{|c|c|c|c|c|c|c|c|c|}
\hline Atom\# & C Shift & H Shift & H Multiplicity & $\mathrm{H}$ Volume & $\cos Y$ & NOESY & H HMBC & C HMBC \\
\hline 19 & -4.320 & & & & & & 0.15 & \\
\hline 19,18 & -3.592 & 0.150 & $d(4.10)$ & 5.992 & & 3.88 & & $18.29,-4.32$ \\
\hline 20 & 18.293 & & & & & & $0.15,0.94$ & \\
\hline 7 & 18.809 & 0.847 & $\mathbf{s}$ & 3.162 & & $4.24,3.88,2$ & $1.57,4.24,4.38$ & $42.98,52.34,38.37,72.94$ \\
\hline $21,22,23$ & 26.286 & 0.940 & s & 8.862 & & & & 18.29 \\
\hline 2 & 28.842 & 1.946 & $\mathrm{~m}$ & -0.979 & $1.52,1.72,3.88$ & 3.88 & & \\
\hline 2 & 28.842 & 1.721 & $\mathrm{~m}$ & -1.051 & $1.95,3.88$ & & & $56.88,72.94,42.98,29.32$ \\
\hline 3 & 29.320 & 1.641 & $\mathrm{~m}$ & -1.255 & 1.52 & & $1.57,1.72,2.58$ & 56.88 \\
\hline 3 & 29.320 & 1.525 & $\mathrm{~m}$ & -1.537 & $1.95,1.64$ & & $1.57,1.72,2.58$ & \\
\hline 5 & 38.374 & 1.171 & brd (13.24) & -1.063 & 1.57 & & $0.85,2.58,4.24$ & \\
\hline 5 & 38.374 & 1.570 & $\operatorname{brd}(14.03)$ & -1.222 & 1.17 & & $0.85,2.58,4.24$ & $56.88,18.81,72.94,42.98,29.32$ \\
\hline 6 & 42.978 & & & & & & $0.85,1.57,1.72,4.24,4.38$ & \\
\hline \multirow[t]{2}{*}{8} & 52.343 & & & & & & 0.85 & \\
\hline & 53.336 & & & & & & & \\
\hline 9 & 53.466 & & & & & & & \\
\hline 4 & 56.878 & & & & & & $1.57,1.64,1.72,2.58$ & \\
\hline 1 & 72.940 & 3.879 & $\operatorname{dd}(7.72,3.31)$ & 0.971 & $1.72,1.95$ & $0.15,0.85,1$ & $0.85,1.57,1.72,4.24$ & \\
\hline 14 & 104.767 & & & & & & & \\
\hline 16 & 116.946 & 8.358 & s & 0.981 & & $4.24,4.38$ & 7.59 & $146.23,125.20,120.34$ \\
\hline 15 & 120.344 & & & & & & $7.59,8.36$ & \\
\hline 12 & 121.125 & 7.831 & $d(8.35)$ & 0.969 & 7.59 & & & 135.53 \\
\hline 13 & 125.205 & 7.591 & $\mathrm{dd}(8.35,1.26)$ & 0.974 & 7.83 & & 8.36 & $146.23,120.34,116.95$ \\
\hline 17 & 135.526 & & & & & & $4.24,4.38,7.83,8.42$ & \\
\hline
\end{tabular}

\begin{tabular}{|c|c|c|c|c|c|c|c|c|}
\hline Atom\# & C Shift & H Shift & H Multiplicity & H Volume & COSY & NOESY & H HMBC \\
\hline 11 & 146.226 & & & & & & $7.59,8.36,8.42$ \\
\hline & 148.946 & & & & & & \\
\hline 10 & 148.971 & & & & & & \\
\hline 8 & & 4.375 & $\mathrm{~d}(14.34)$ & 1.006 & 4.24 & $3.88,8.42,8.24,4.38$ & \\
\hline & & 8.481 & $\mathrm{u}$ & 0.003 & & & \\
\hline 10 & & 8.418 & $\mathrm{~s}$ & -1.000 & & $4.24,4.38$ & \\
\hline 8 & & 4.245 & $\mathrm{~d}(14.34)$ & -0.958 & 4.38 & $0.85,3.88,8$. & \\
\hline 9 & & 2.576 & $\mathrm{~m}$ & 1.925 & & 0.85 & \\
\hline
\end{tabular}
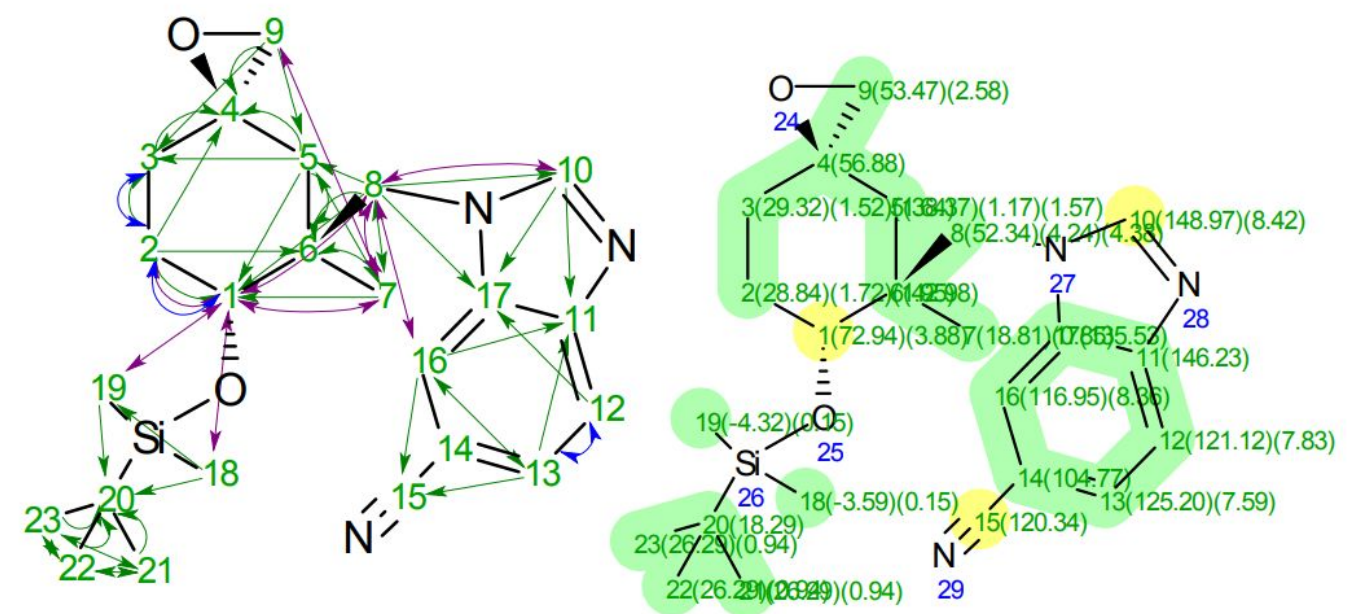

Experimentally Observed Proton and Carbon-13 Shifts

Color represents calculation agreement 

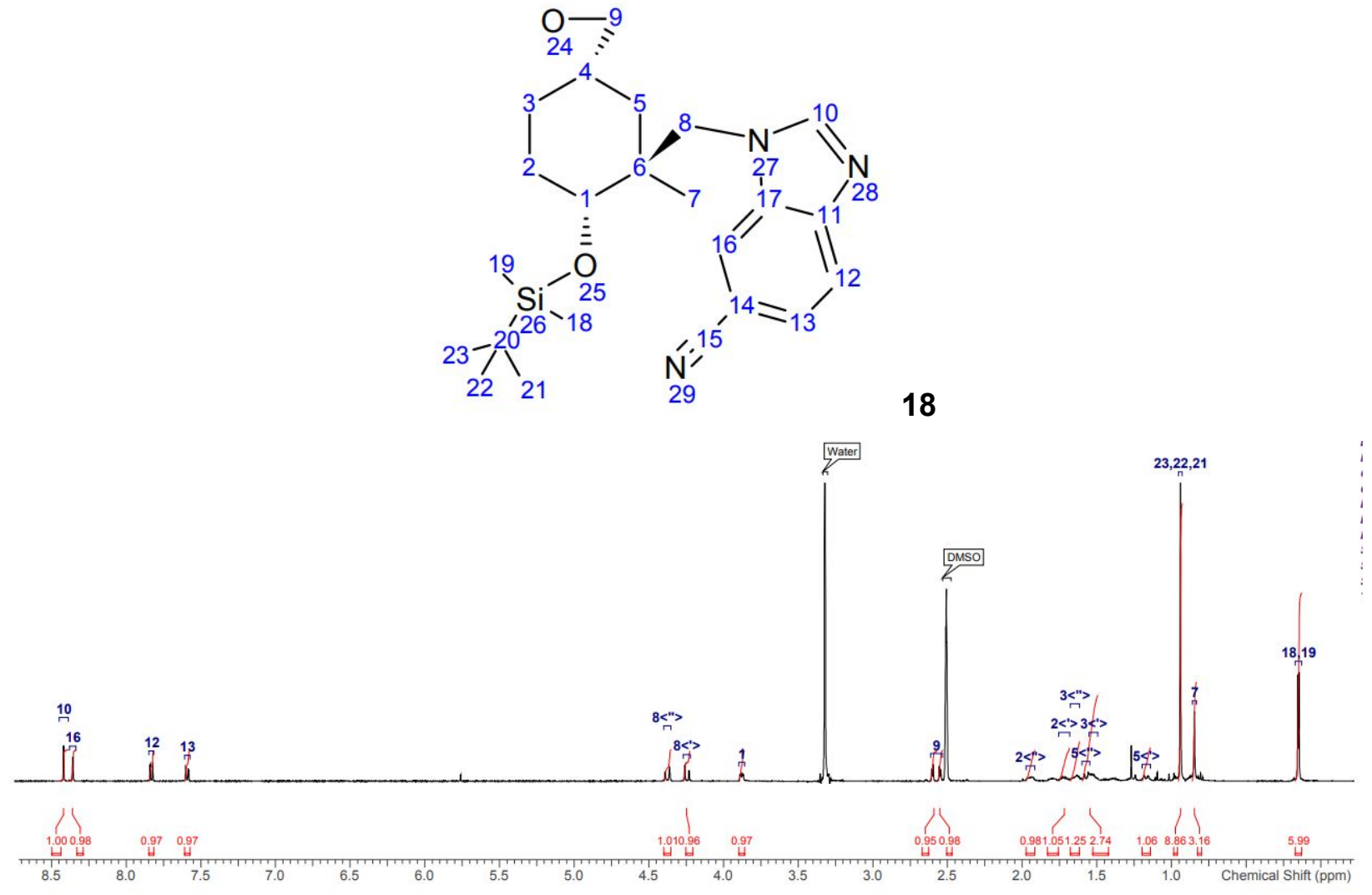

${ }^{1} \mathrm{H}$ NMR (DMSO-d $\left.\mathrm{d}_{6}, 500 \mathrm{MHz}\right): \delta(\mathrm{ppm}) 8.42(\mathrm{~s}, 1 \mathrm{H}), 8.36(\mathrm{~s}, 1 \mathrm{H}), 7.83$ (d, J=8.4 Hz, 1H), 7.59 (dd, J=8.4, 1.3 Hz, 1H), $4.38(\mathrm{~d}, \mathrm{~J}=14.3 \mathrm{~Hz}, 1 \mathrm{H}), 4.24(\mathrm{~d}, \mathrm{~J}=14.3 \mathrm{~Hz}, 1 \mathrm{H}), 3.88(\mathrm{dd}, \mathrm{J}=7.7,3.3 \mathrm{~Hz}$,

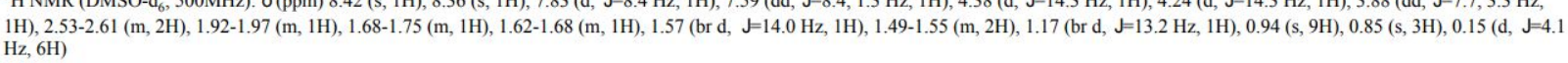

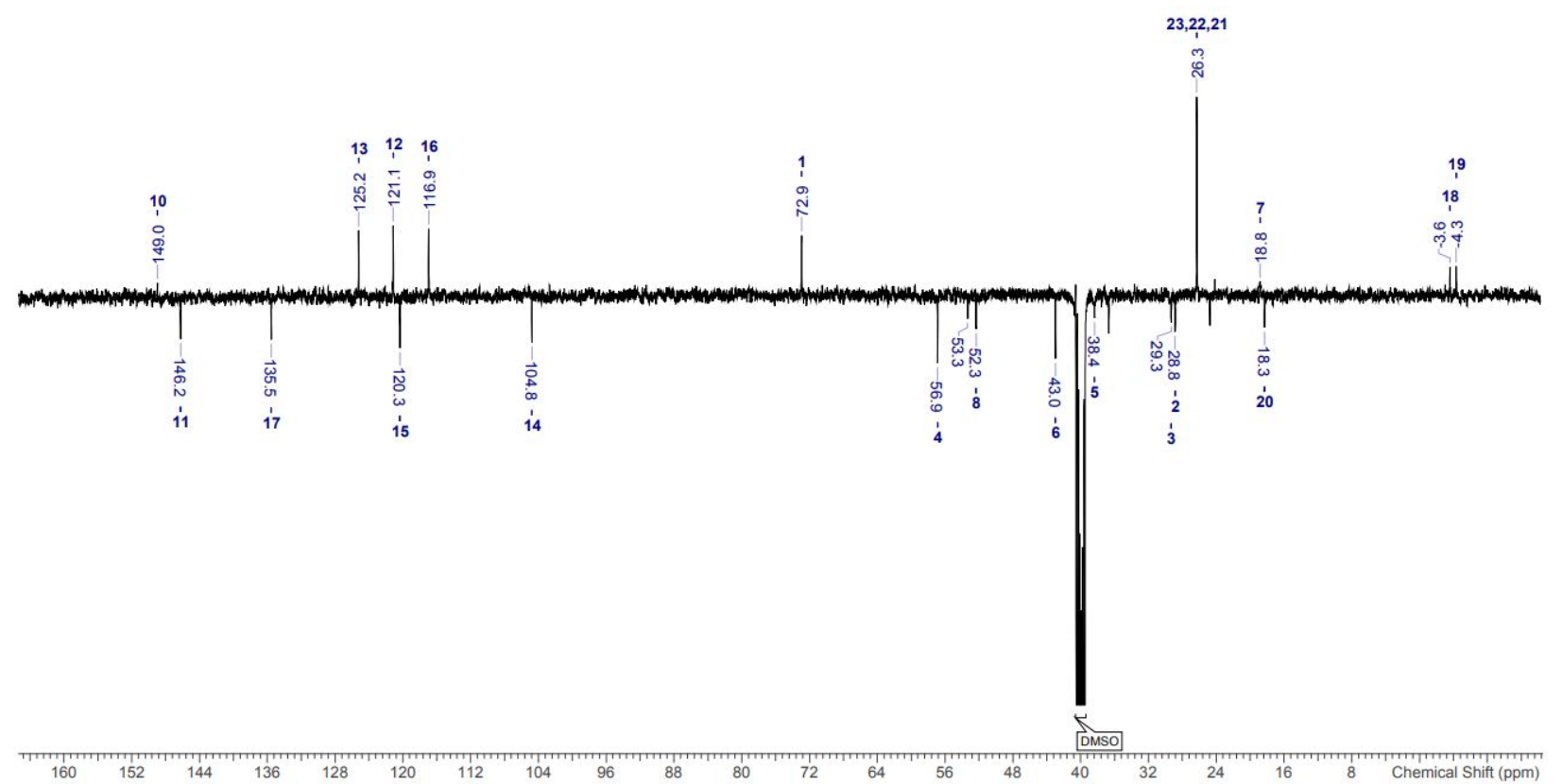

${ }^{13} \mathrm{C}$ NMR (DMSO-d 6 , 126MHz): $\delta$ (ppm) 149.0, 146.2, 135.5, 125.2, 121.1, 120.3, 116.9, 104.8, 72.9, 56.9, 53.3, 52.3, 43.0, 38.4, 29.3, 28.8, 26.3, 18.8, 18.3, -3.6, -4.3 


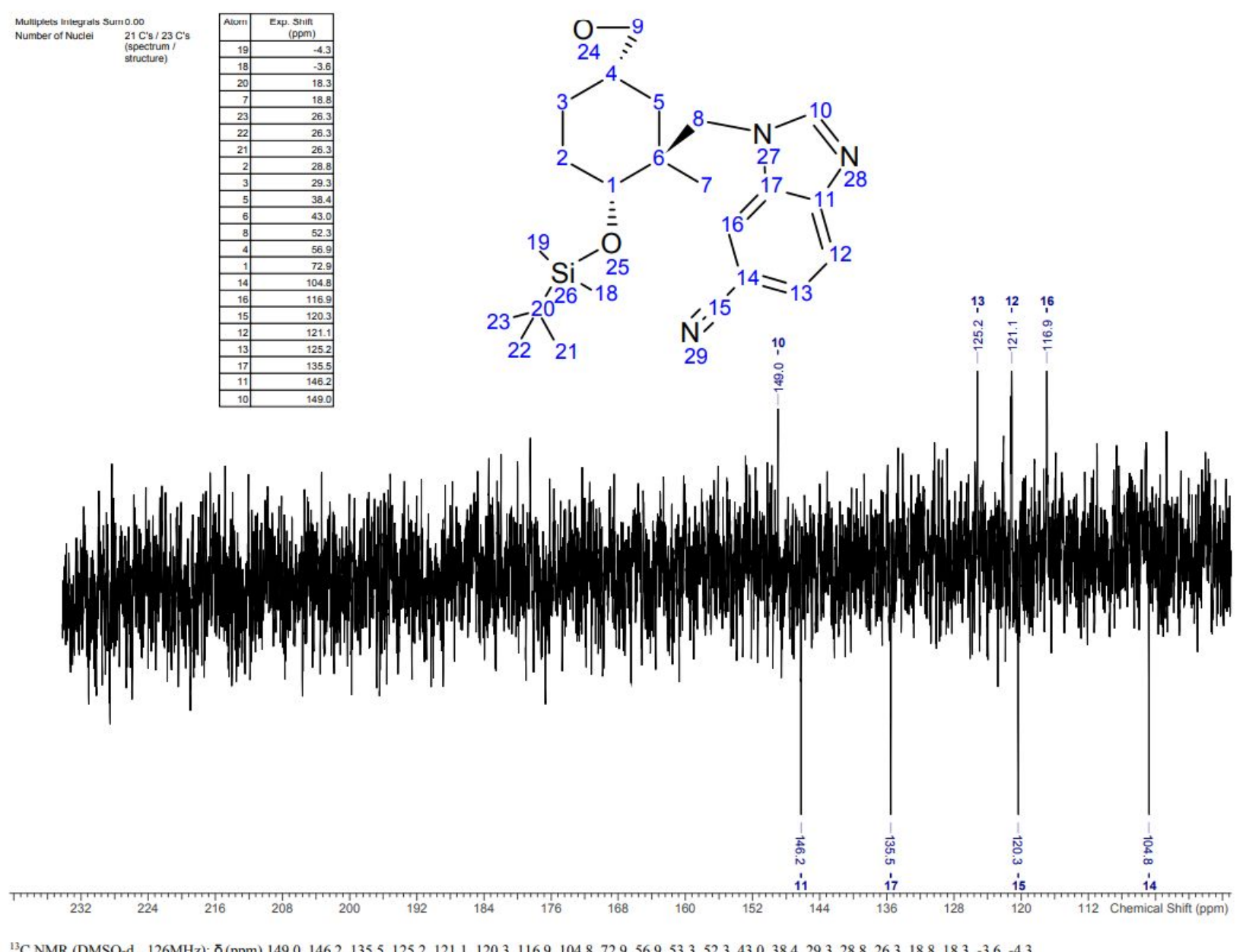




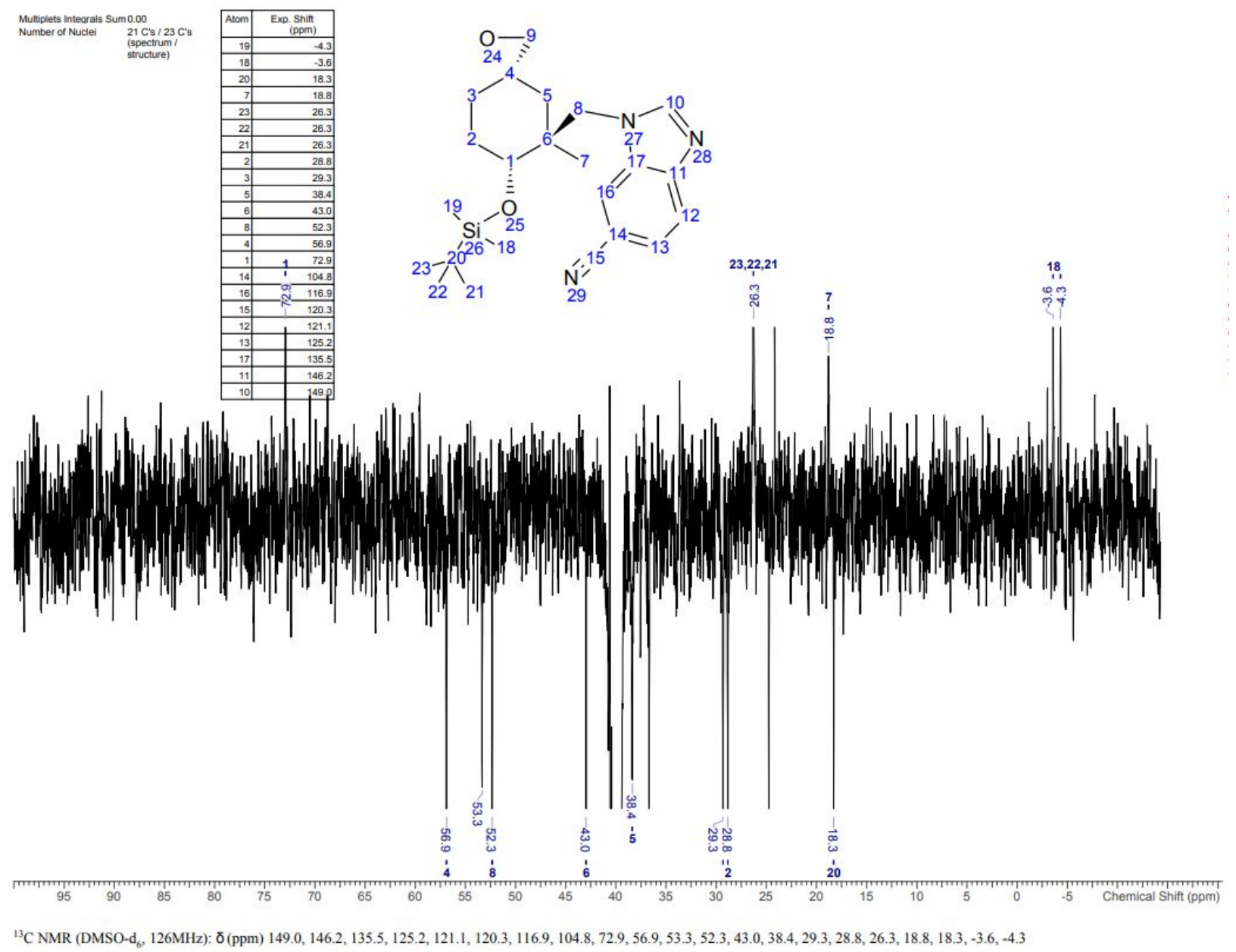




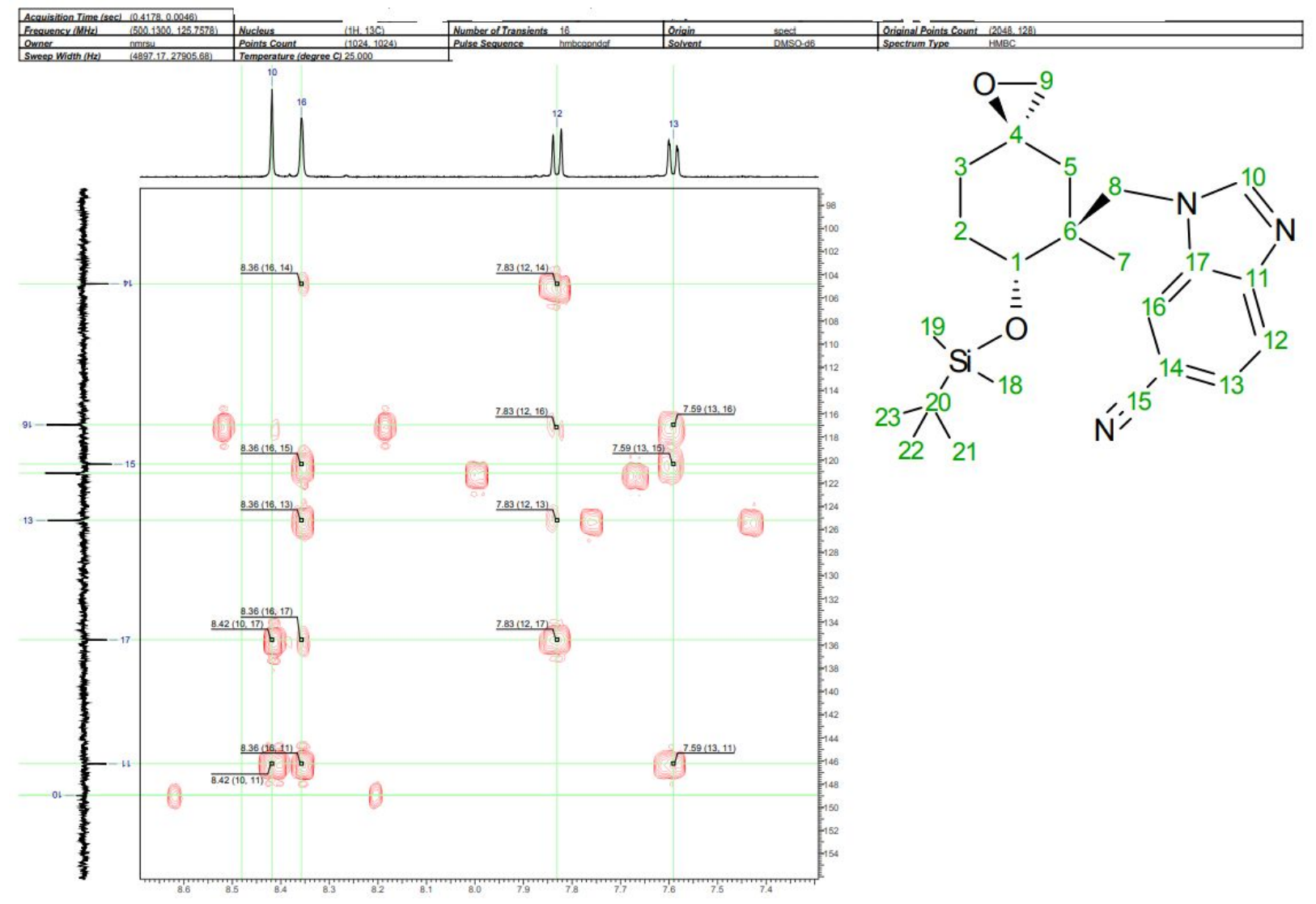




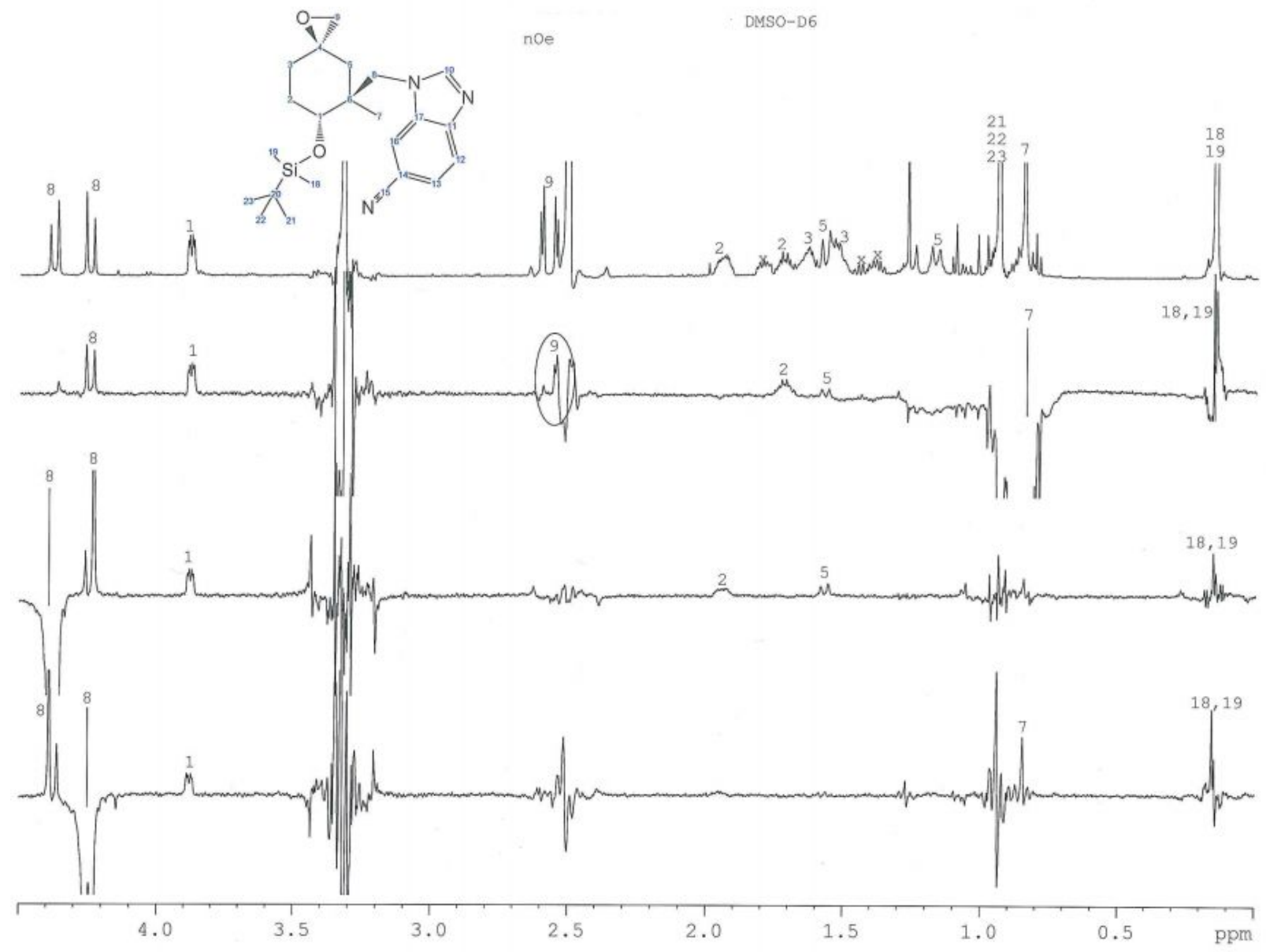




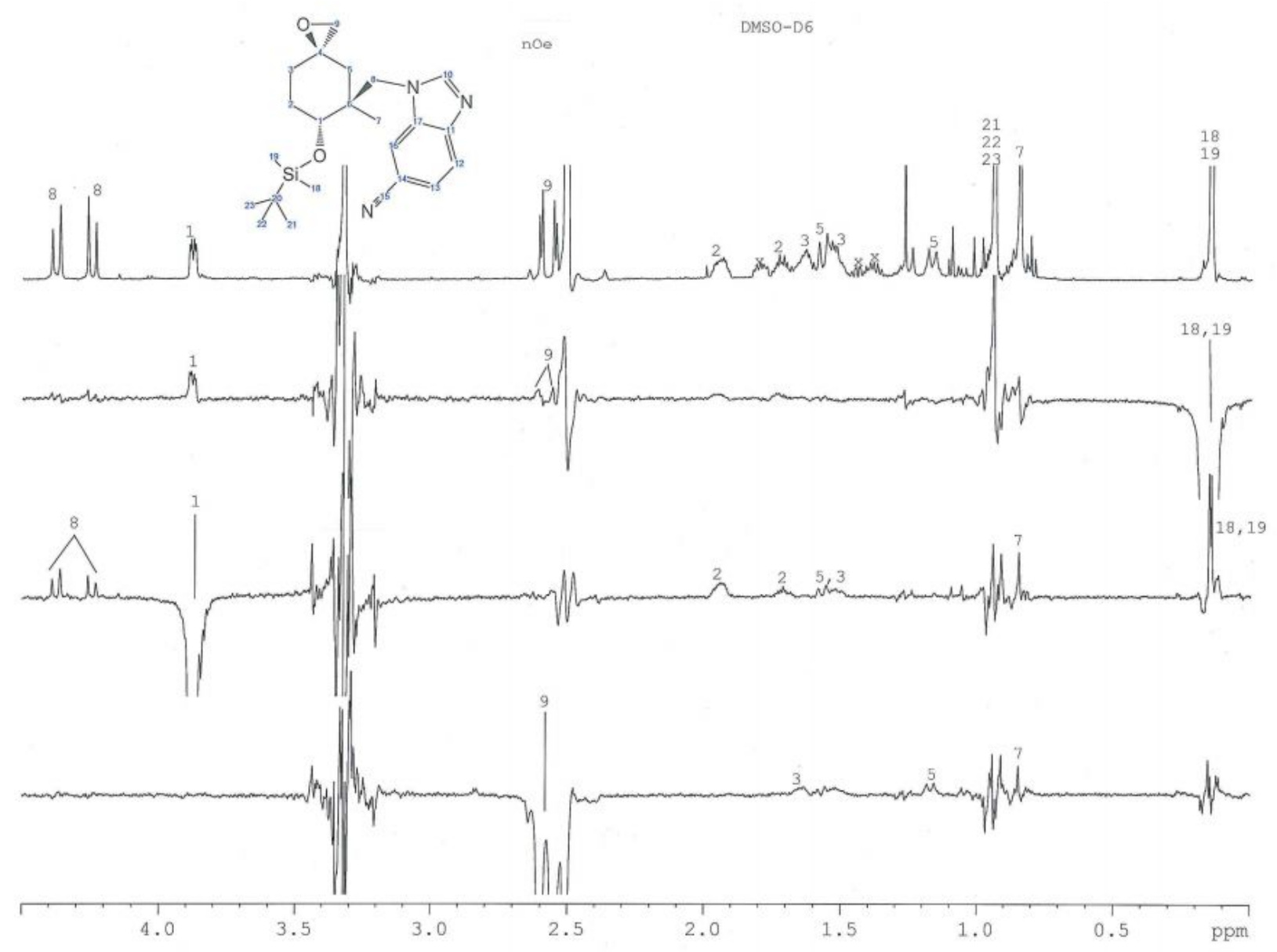

1-(((5S,7S,8R)-8-((tert-Butyldimethylsilyl)oxy)-7-methyl-2-oxo-1-oxa-3-azaspiro[4.5]decan7-yl)methyl)-1H-benzo[d]imidazole-6-carbonitrile (9)

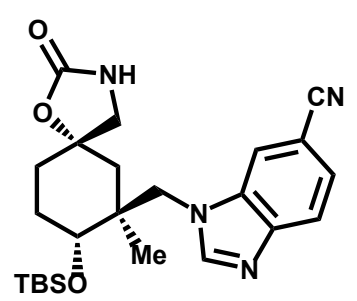

Lithium tert-butoxide (1.0 M in hexanes) $(222 \mu \mathrm{l}, 0.222 \mathrm{mmol})$ was added to a solution of ethyl carbamate (198 mg, $2.221 \mathrm{mmol}$ ) in $N$-methyl-2- pyrrolidone (NMP) $(555 \mu \mathrm{L})$. After stirring for 5 $\min$ at RT, a solution of 1-(((3S,5S,6R)-6-((tert-butyldimethylsilyl)oxy)-5-methyl-1oxaspiro[2.5]octan-5-yl)methyl)-1H-benzo[d]imidazole-6-carbonitrile (45.7 mg, $0.111 \mathrm{mmol})$ in $\mathrm{N}$ methyl-2-pyrrolidone (NMP) $(555 \mu \mathrm{L})$ was added dropwise, giving a clear, golden solution. The reaction mixture was subsequently heated to $100{ }^{\circ} \mathrm{C}$. After $16.5 \mathrm{~h}$, the reaction mixture was cooled to RT and directly purified by reverse-phase HPLC (10-90\% $\mathrm{CH}_{3} \mathrm{CN}: \mathrm{H}_{2} \mathrm{O}, 0.1 \%$ TFA as modifier). 
The product-containing fractions were partitioned with EtOAc and saturated aqueous $\mathrm{NaHCO}_{3}$ and the layers were separated. The aqueous layer was extracted with EtOAc $(3 \times 10 \mathrm{~mL})$. The combined organics were dried over anhydrous $\mathrm{Na}_{2} \mathrm{SO}_{4}$, filtered and concentrated in vacuo to give the title compound as a white solid ( $38.0 \mathrm{mg}, 0.084 \mathrm{mmol}, 75 \%$ yield). LC-MS $\mathrm{C}_{24} \mathrm{H}_{34} \mathrm{~N}_{4} \mathrm{O}_{3} \mathrm{Si}[\mathrm{M}+\mathrm{H}]$ calculated: 455.2, observed: 454.7, $1.13 \mathrm{~min}$ (ret. time); ${ }^{1} \mathrm{H}$ NMR $\left(400 \mathrm{MHz}, \mathrm{CD}_{3} \mathrm{OD}\right) \delta \mathrm{ppm} 0.23$ $(\mathrm{s}, 3 \mathrm{H}) 0.26(\mathrm{~s}, 3 \mathrm{H}) 1.06(\mathrm{~s}, 9 \mathrm{H}) 1.18(\mathrm{~s}, 3 \mathrm{H}) 1.67(\mathrm{~s}, 2 \mathrm{H}) 1.73$ - $1.88(\mathrm{~m}, 2 \mathrm{H}) 1.90$ - $2.01(\mathrm{~m}$, $1 \mathrm{H}) 2.04-2.09(\mathrm{~m}, 1 \mathrm{H}) 3.20(\mathrm{~s}, 1 \mathrm{H}) 3.24(\mathrm{~s}, 1 \mathrm{H}) 3.77-3.85(\mathrm{~m}, 1 \mathrm{H}) 4.28(\mathrm{~s}, 1 \mathrm{H}) 4.34(\mathrm{~s}, 1 \mathrm{H})$ $7.63(\mathrm{~s}, 1 \mathrm{H}) 7.85(\mathrm{~s}, 1 \mathrm{H}) 8.10(\mathrm{~s}, 1 \mathrm{H}) 8.37(\mathrm{~s}, 1 \mathrm{H})$.

\section{1-(((5S,7S,8R)-8-((tert-Butyldimethylsilyl)oxy)-3-(5-(2-hydroxypropan-2-yl)pyrazin-2-yl)-7-} methyl-2-oxo-1-oxa-3-azaspiro[4.5]decan-7-yl)methyl)-1H-benzo[d]imidazole-6carbonitrile

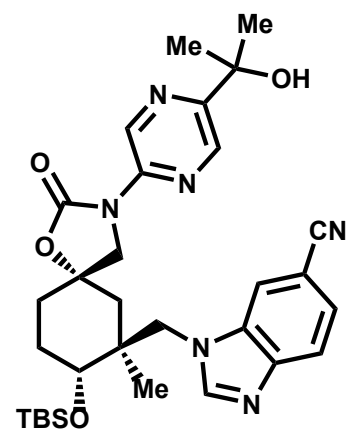

To a solution of 1-(((5S,7S,8R)-8-((tert-butyldimethylsilyl)oxy)-7-methyl-2-oxo-1-oxa-3azaspiro[4.5]decan-7-yl)methyl)-1H-benzo[d]imidazole6-carbonitrile (37.3 $\mathrm{mg}, 0.082 \mathrm{mmol})$ in 1,4-dioxane $(456 \mu \mathrm{L})$ was added potassium phosphate tribasic $(34.8 \mathrm{mg}, 0.164 \mathrm{mmol})$ and copper(I) iodide $(15.63 \mathrm{mg}, 0.082 \mathrm{mmol})$, giving a beige slurry. The reaction mixture was warmed to $100^{\circ} \mathrm{C}$ after which $N 1, N 2-$ dimethylethane-1,2-diamine $(17.7 \mu \mathrm{L}, 0.164 \mathrm{mmol})$ was added, giving a clear, pale green solution. A solution of 2-(5-bromopyrazin-2-yl)propan-2-ol (26.7 mg, 0.123 mmol) in 1,4-dioxane $(91 \mu \mathrm{L})$ was subsequently added and the reaction mixture turned dark green/brown in color. After $60 \mathrm{~min}$ at $100^{\circ} \mathrm{C}$, the reaction mixture was diluted with $5 \mathrm{~mL} \mathrm{DCM}$ and partitioned with $5 \mathrm{~mL}$ of $1: 1 \mathrm{NH}_{4} \mathrm{OH}: \mathrm{H}_{2} \mathrm{O}$. The resulting layers were separated and the organic layer was washed with 1:1 $\mathrm{NH}_{4} \mathrm{OH}: \mathrm{H}_{2} \mathrm{O}(4 \times 5 \mathrm{~mL})$. The organics were dried over anhydrous $\mathrm{Na}_{2} \mathrm{SO}_{4}$, filtered and concentrated to give a clear, orange oil. The crude product was purified by silica gel chromatography (12 g RediSep Gold column, 0-100\% EtOAc:Hexane) to give the title compound as a pale yellow solid (35.7 mg, $0.060 \mathrm{mmol}, 74 \%$ yield). LC-MS $\mathrm{C}_{31} \mathrm{H}_{42} \mathrm{~N}_{6} \mathrm{O}_{4} \mathrm{SiNa}$ [M+Na], calculated: 613.3, observed: 613.2, $1.29 \mathrm{~min}$ (ret. time); ${ }^{1} \mathrm{H}$ NMR (400 $\left.\mathrm{MHz}, \mathrm{CD}_{3} \mathrm{OD}\right) \delta$ ppm 0.25 (s, 3 H) $0.28(\mathrm{~s}, 3 \mathrm{H}) 1.07$ (s, $9 \mathrm{H}) 1.22$ (s, $3 \mathrm{H}) 1.55(\mathrm{~s}, 6 \mathrm{H}) 1.72$ - 1.95 (m, 5 H) 2.07 - 
$2.17(\mathrm{~m}, 1 \mathrm{H}) 3.86(\mathrm{~s}, 2 \mathrm{H}) 3.91-3.97(\mathrm{~m}, 1 \mathrm{H}) 4.23$ - $4.33(\mathrm{~m}, 1 \mathrm{H}) 4.35$ - $4.43(\mathrm{~m}, 1 \mathrm{H}) 7.56$ $7.65(\mathrm{~m}, 1 \mathrm{H}) 7.78$ - $7.88(\mathrm{~m}, 1 \mathrm{H}) 8.10(\mathrm{~s}, 1 \mathrm{H}) 8.38(\mathrm{~s}, 1 \mathrm{H}) 8.59(\mathrm{~s}, 1 \mathrm{H}) 9.26(\mathrm{~s}, 1 \mathrm{H})$.

1-(((5S,7S,8R)-8-Hydroxy-3-(5-(2-hydroxypropan-2-yl)pyrazin-2-yl)-7-methyl-2-oxo-1-oxa3-azaspiro[4.5]decan-7-yl)methyl)-1H-benzo[d]imidazole-6-carbonitrile (1) 1-(((5R,7R,8S)-8-Hydroxy-3-(5-(2-hydroxypropan-2-yl)pyrazin-2-yl)-7-methyl-2-oxo-1-oxa3-azaspiro[4.5]decan-7-yl)methyl)-1H-benzo[d]imidazole-6-carbonitrile ((ent)-1)

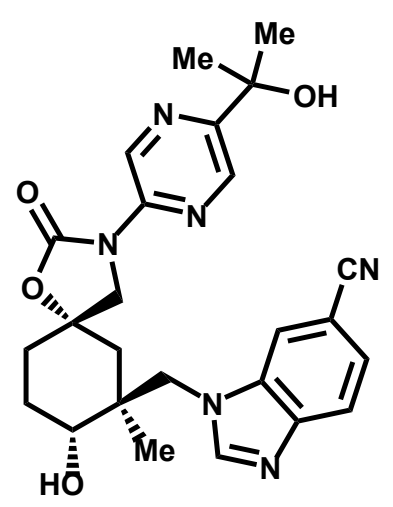

1

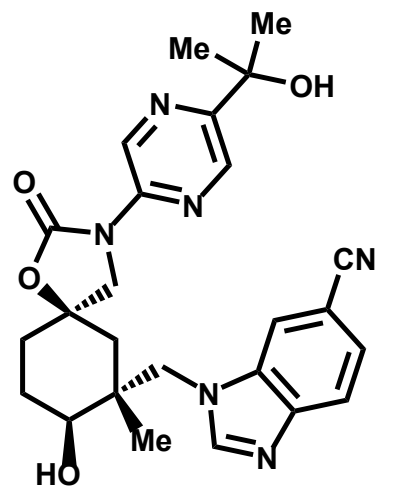

(ent)-1

TBAF (266 $\mu \mathrm{L}, 0.266 \mathrm{mmol})$ was added to a solution of 1-(((5S,7S,8R)-8-((tertbutyldimethylsilyl)oxy)-3-(5-(2-hydroxypropan-2-yl)pyrazin-2-yl)-7-methyl-2-oxo-1-oxa-3azaspiro[4.5]decan-7-yl)methyl)-1H-benzo[d]imidazole-6-carbonitrile (52.3 mg, $0.089 \mathrm{mmol})$ in tetrahydrofuran (THF) (2.2 mL) at RT. After $90 \mathrm{~min}$, the reaction contents were partitioned with 20 $\mathrm{mL}$ of EtOAc and $15 \mathrm{~mL}$ of saturated aqueous $\mathrm{NaHCO}_{3}$ and the layers were separated. The organic layer was washed with saturated aqueous $\mathrm{NaHCO}_{3}(1 \times 15 \mathrm{~mL})$. The combined aqueous layers were back-extracted with EtOAc $(3 \times 10 \mathrm{~mL})$. The combined organic layers were dried over anhydrous $\mathrm{Na}_{2} \mathrm{SO}_{4}$, filtered and concentrated in vacuo to give an off-white solid. The crude material was dissolved in $0.5 \mathrm{~mL} \mathrm{MeOH}$ and purified by reverse-phase HPLC (10-70\% $\mathrm{CH}_{3} \mathrm{CN}: \mathrm{H}_{2} \mathrm{O}, 0.1 \%$ TFA as modifier). The product-containing fractions were partitioned with EtOAc and saturated aqueous $\mathrm{NaHCO}_{3}$ and the layers were separated. The aqueous layer was extracted with EtOAc $(3 \times 10 \mathrm{~mL})$. The combined organics were dried over anhydrous $\mathrm{Na}_{2} \mathrm{SO}_{4}$, filtered and concentrated in vacuo to give a white solid $(38.1 \mathrm{mg})$.

Chiral analytical HPLC (Chiralcel OD-H, 5 H, 4.6 x 100 mm, 100:0.1 MeOH:isopropylamine, 1.0 $\mathrm{mL} / \mathrm{min}$ flow rate) revealed an 88:11 ratio of enantiomers 1 and (ent)-1. 
Preparative HPLC to isolate 1 and (ent)-1: Chiralcel OD-H $(5 \mu, 20 \times 250 \mathrm{~mm}, \mathrm{MeOH}, 20 \mathrm{~mL} / \mathrm{min}$ flow rate) afforded:

1: $32.0 \mathrm{mg}$ (white solid, $0.067 \mathrm{mmol}, 76 \%$ yield); LC-MS $\mathrm{C}_{25} \mathrm{H}_{28} \mathrm{~N}_{6} \mathrm{O}_{4} \mathrm{Na}$ [M+Na] calculated: 499.2, observed: 499.5, $0.66 \mathrm{~min}$ (ret. time); ${ }^{1} \mathrm{H}$ NMR $\left(\mathrm{CD}_{3} \mathrm{OD}, 500 \mathrm{MHz}\right): \delta(\mathrm{ppm}) 9.31$ (d, J=1.4 Hz, $\left.1 \mathrm{H}\right)$, $8.60(\mathrm{~d}, J=1.6 \mathrm{~Hz}, 1 \mathrm{H}), 8.31-8.54(\mathrm{~m}, 1 \mathrm{H}), 8.29$ (d, J=0.8 Hz, 1H), $7.84(\mathrm{~d}, J=8.4 \mathrm{~Hz}, 1 \mathrm{H}), 7.61$ (dd, J=8.4, $1.4 \mathrm{~Hz}, 1 \mathrm{H}$ ), 4.45 (d, J=14.7 Hz, 1H), 4.20 (d, J=14.8 Hz, 1H), 3.88 (s, 2H), 3.45 (dd, $J=11.4,4.2 \mathrm{~Hz}, 1 \mathrm{H}), 2.08-2.13(\mathrm{~m}, 1 \mathrm{H}), 2.04-2.09(\mathrm{~m}, 1 \mathrm{H}), 1.96-2.03(\mathrm{~m}, 1 \mathrm{H}), 1.77-1.82(\mathrm{~m}, 1 \mathrm{H})$, $1.69(\mathrm{br} \mathrm{d}, J=3.2 \mathrm{~Hz}, 1 \mathrm{H}), 1.60-1.65(\mathrm{~m}, 1 \mathrm{H}), 1.66(\mathrm{br} \mathrm{d}, J=4.1 \mathrm{~Hz}, 1 \mathrm{H}), 1.56(\mathrm{~d}, J=3.0 \mathrm{~Hz}, 6 \mathrm{H})$, $1.28(\mathrm{~s}, 3 \mathrm{H}) ; 100 \%$ ee by chiral analytical HPLC; $[\alpha]_{\mathrm{D}}=+24^{\circ}(\mathrm{c}=0.1, \mathrm{MeOH})$.

(ent)-1: $3.5 \mathrm{mg}$ (white solid, $7.3 \mu \mathrm{mol}, 8.3 \%$ yield); LC-MS $\mathrm{C}_{25} \mathrm{H}_{28} \mathrm{~N}_{6} \mathrm{O}_{4} \mathrm{Na}$ [M+Na] calculated: 499.2, observed: 499.5, 0.66 min (ret. time); $96.6 \%$ ee by chiral analytical HPLC.

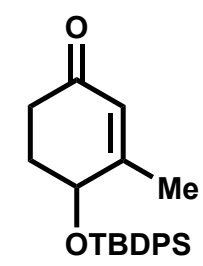

\section{4-((tert-Butyldiphenylsilyl)oxy)-3-methylcyclohex-2-en-1-one (racemate, 10)}

1-Methyl-7-oxabicyclo[4.1.0]heptan-3-one (51 g, $403 \mathrm{mmol})$ as a solution in $500 \mathrm{~mL}$ of dichloromethane was treated with diisopropylethylamine $(148 \mathrm{~mL}, 846 \mathrm{mmol})$, tertbutyldiphenylsilyl chloride (104 mL, $403 \mathrm{mmol})$, and DMAP $(9.9 \mathrm{~g}, 81 \mathrm{mmol})$ at room temperature under an atmosphere of nitrogen. The reaction mixture was allowed to stir for $72 \mathrm{~h}$, after which HPLC (Eclipse XDB-C18, 4.6 x 250 mm, 5 micron, $2.5 \mathrm{~mL} / \mathrm{min}$, gradient from 1-99\% $\mathrm{CH}_{3} \mathrm{CN}(0.1$ $\%$ trifluoroacetic acid $) / \mathrm{H}_{2} \mathrm{O}(0.1 \%$ trifluoroacetic acid $), 10$ minutes plus 2 minute plateau at 99 $\% \mathrm{CH}_{3} \mathrm{CN}(0.1 \%$ trifluoroacetic acid)) and LCMS (SunFire, C18, $5 \mu \mathrm{m}, 3.0 \times 50 \mathrm{~mm}$, gradient 10 - $100 \% \mathrm{CH}_{3} \mathrm{CN}$ in $\mathrm{H}_{2} \mathrm{O}$ with $0.1 \%$ TFA, $2.5 \mathrm{~min}$ ) indicated that the reaction was complete. The reaction mixture was poured into $2 \mathrm{~L}$ of EtOAc and $1 \mathrm{~L}$ of water and the layers were separated. The organic layer was washed with water, dried over anhydrous sodium sulfate, filtered and concentrated to give a light yellow oil. The material was purified by silica gel column chromatography to provide the title compound as a light yellow oil (120 g) which was mixed with some tert-butyldiphenylsilanol byproduct, but was carried on to the next step without further purification. 


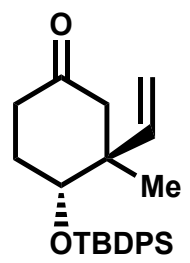

Copper(I) bromide-dimethyl sulfide complex $(37.2 \mathrm{~g}, 181 \mathrm{mmol})$ was suspended in tetrahydrofuran (THF) $(1 \mathrm{~L})$ under an atmosphere of nitrogen and the reaction mixture was cooled to $-78{ }^{\circ} \mathrm{C}$. Vinylmagnesium bromide $(543 \mathrm{~mL}, 543 \mathrm{mmol})$ was then added dropwise. Upon completion of the addition, the reaction mixture was stirred at $-78{ }^{\circ} \mathrm{C}$ for one hour. 4((tert-Butyldiphenylsilyl)oxy)-3-methylcyclohex-2-en-1-one (66 g, $181 \mathrm{mmol}$ ) was added dropwise as a solution in $200 \mathrm{~mL}$ of THF. The reaction mixture was allowed to stir for one hour after which HPLC (Eclipse XDB-C18, 4.6 x 250 mm, 5 micron, $2.5 \mathrm{~mL} / \mathrm{min}$, gradient from 1-99\% $\mathrm{CH}_{3} \mathrm{CN}(0.1$ $\%$ trifluoroacetic acid) $/ \mathrm{H}_{2} \mathrm{O}(0.1 \%$ trifluoroacetic acid $), 10$ minutes plus 2 minute plateau at 99 $\% \mathrm{CH}_{3} \mathrm{CN}(0.1 \%$ trifluoroacetic acid)) and LCMS (SunFire, C18, $5 \mu \mathrm{m}, 3.0 \times 50 \mathrm{~mm}$, gradient 10 - $100 \% \mathrm{CH}_{3} \mathrm{CN}$ in $\mathrm{H}_{2} \mathrm{O}$ with $0.1 \%$ TFA, $2.5 \mathrm{~min}$ ) indicated that the reaction was complete. The reaction mixture was quenched at $-78^{\circ} \mathrm{C}$ with $100 \mathrm{~mL}$ of saturated aqueous ammonium chloride. The reaction mixture was then poured into $2 \mathrm{~L}$ of ethyl acetate and $2 \mathrm{~L}$ of water. The layers were separated and the organic phase was washed with water $(3 \times 2 L)$, dried over anhydrous sodium sulfate, filtered and concentrated to provide 4-((tert-butyldiphenylsilyl)oxy)-3-methyl-3vinylcyclohexanone racemate $(70 \mathrm{~g}, 143 \mathrm{mmol}, 79 \%$ yield, $>20: 1 \mathrm{dr})$ as a light yellow oil. ${ }^{1} \mathrm{H}$ NMR (400 MHz, DMSO- $\left.d_{6}\right) \delta$ ppm 0.99 - $1.01(\mathrm{~m}, 3 \mathrm{H}) 1.05(\mathrm{~s}, 9 \mathrm{H}) 1.60$ - $1.71(\mathrm{~m}, 2 \mathrm{H}) 2.00$ - 2.07 (m, $1 \mathrm{H}) 2.24$ - $2.31(\mathrm{~m}, 1 \mathrm{H}) 2.34$ - $2.38(\mathrm{~m}, 2 \mathrm{H}) 3.92$ - $3.98(\mathrm{~m}, 1 \mathrm{H}) 4.88$ - $5.03(\mathrm{~m}, 2 \mathrm{H}) 5.65$ - 5.78 $(\mathrm{m}, 1 \mathrm{H}) 7.38$ - $7.40(\mathrm{~m}, 2 \mathrm{H}) 7.46-7.49(\mathrm{~m}, 2 \mathrm{H}) 7.63$ - $7.66(\mathrm{~m}, 2 \mathrm{H}) 7.67$ - $7.71(\mathrm{~m}, 4 \mathrm{H})$.

\section{tert-Butyl(((3S,5S,6R / 3R,5R,6S)-5-methyl-5-vinyl-1-oxaspiro[2.5]octan-6-yl)oxy)-} diphenylsilane (racemate, 12)

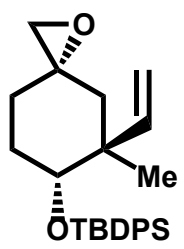

Trimethylsulfoxonium iodide (157 g, $713 \mathrm{mmol}$ ) was suspended in $N, N$-dimethylformamide (DMF) $(500 \mathrm{~mL})$ under an atmosphere of nitrogen and cooled to $0{ }^{\circ} \mathrm{C}$. The reaction mixture was then treated with potassium tert-butoxide $(80 \mathrm{~g}, 713 \mathrm{mmol})$, allowed to warm to room temperature and 
stirred for 30 minutes in order to ensure that formation of the ylide was complete. The reaction mixture was then cooled to $-60{ }^{\circ} \mathrm{C}$ and treated with a solution of $(3 S, 4 R / 3 R, 4 S)-4-(($ tertbutyldiphenylsilyl)oxy)-3-methyl-3-vinylcyclohexanone racemate $(70 \mathrm{~g}, 178 \mathrm{mmol})$ in $100 \mathrm{~mL}$ of DMF.

The reaction mixture was allowed to warm slowly to room temperature and stirred for 90 minutes, after which HPLC (Eclipse XDB-C18, $4.6 \times 250 \mathrm{~mm}, 5$ micron, $2.5 \mathrm{~mL} / \mathrm{min}$, gradient from 1-99\% $\mathrm{CH}_{3} \mathrm{CN}\left(0.1 \%\right.$ trifluoroacetic acid) / $\mathrm{H}_{2} \mathrm{O}(0.1 \%$ trifluoroacetic acid $), 10$ minutes plus 2 minute plateau at $99 \% \mathrm{CH}_{3} \mathrm{CN}(0.1 \%$ trifluoroacetic acid)) and LCMS (SunFire, C18, $5 \mu \mathrm{m}, 3.0 \times 50 \mathrm{~mm}$, gradient $10-100 \% \mathrm{CH}_{3} \mathrm{CN}$ in $\mathrm{H} 2 \mathrm{O}$ with $0.1 \%$ TFA, $2.5 \mathrm{~min}$ ) indicated that the reaction was complete. The reaction mixture was poured into $2 \mathrm{~L}$ of ether and $2 \mathrm{~L}$ of water. The layers were separated and the organic layer was washed with water $(3 \times 2 L)$. The organic layer was dried over anhydrous sodium sulfate, filtered and concentrated to give tert-butyl(((3S,5S,6R / $3 R, 5 R, 6 S$ )-5-methyl-5-vinyl-1 oxaspiro[2.5]octan-6-yl)oxy)diphenylsilane racemate (59 g, 116 mmol, $65 \%$ yield, $7: 1 \mathrm{dr}$ ) as a pale yellow oil which was used in the next step without further purification. LC-MS $\mathrm{C}_{26} \mathrm{H}_{34} \mathrm{O}_{2} \mathrm{SiNa}$ [M+Na] calculated: 429.2, observed: 429.2, 2.37 min (ret. time); ${ }^{1} \mathrm{H}$ NMR (400 MHz, DMSO-d $\left.d_{6}\right) \delta$ ppm $1.01(\mathrm{~s}, 9 \mathrm{H}) 1.13-1.15(\mathrm{~m}, 3 \mathrm{H}) 1.21-1.27(\mathrm{~m}, 2 \mathrm{H}) 1.40$ - $1.48(\mathrm{~m}, 2 \mathrm{H}) 1.58-1.66(\mathrm{~m}, 1 \mathrm{H}) 1.67-1.74(\mathrm{~m}, 1 \mathrm{H}) 2.41-2.44(\mathrm{~m}, 1 \mathrm{H}) 2.45-2.47(\mathrm{~m}, 1 \mathrm{H})$ $3.66-3.73(\mathrm{~m}, 1 \mathrm{H}) 4.93-4.96(\mathrm{~m}, 1 \mathrm{H}) 4.96-5.01(\mathrm{~m}, 1 \mathrm{H}) 5.80-5.92(\mathrm{~m}, 1 \mathrm{H}) 7.40(\mathrm{~s}, 2 \mathrm{H})$ $7.45-7.48(\mathrm{~m}, 2 \mathrm{H}) 7.62$ - $7.68(\mathrm{~m}, 4 \mathrm{H}) 7.69-7.72(\mathrm{~m}, 2 \mathrm{H})$.

$(5 S, 7 S, 8 R / 5 R, 7 R, 8 S)-8$-((tert-Butyldiphenylsilyl)oxy)-7-methyl-7-vinyl-1-oxa-3azaspiro[4.5]decan-2-one (racemate, 13)

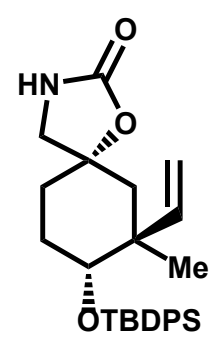

tert-Butyl(((3S,5S,6R / 3R,5R,6S)-5-methyl-5-vinyl-1-oxaspiro[2.5]octan-6-yl)oxy)diphenylsilane $(30 \mathrm{~g}, 73.8 \mathrm{mmol}$ ) racemate was dissolved in $\mathrm{N}$-methyl-2-pyrrolidone (NMP) $(500 \mathrm{~mL})$ and treated with a solution of sodium cyanate $(96 \mathrm{~g}, 1476 \mathrm{mmol})$ in water $(133 \mathrm{~mL}, 7378 \mathrm{mmol})$ at room temperature. The reaction mixture was heated to $140{ }^{\circ} \mathrm{C}$ and stirred for $18 \mathrm{~h}$, after which HPLC (Eclipse XDB-C18, 4.6 × $250 \mathrm{~mm}, 5$ micron, $2.5 \mathrm{~mL} / \mathrm{min}$, gradient from 1-99\% $\mathrm{CH}_{3} \mathrm{CN}(0.1 \%$ trifluoroacetic acid) / $\mathrm{H}_{2} \mathrm{O}$ (0.1\% trifluoroacetic acid), 10 minutes plus 2 minute plateau at $99 \%$ $\mathrm{CH}_{3} \mathrm{CN}(0.1 \%$ trifluoroacetic acid)) and LCMS (SunFire, C18, $5 \mu \mathrm{m}, 3.0 \times 50 \mathrm{~mm}$, gradient 10 - 
$100 \% \mathrm{CH}_{3} \mathrm{CN}$ in $\mathrm{H}_{2} \mathrm{O}$ with $0.1 \%$ TFA, 2.5 min) indicated that the reaction was complete. The reaction mixture was cooled to room temperature and poured into $2 \mathrm{~L}$ of water and $2 \mathrm{~L}$ of a 50 / 50 mixture of ethyl acetate and hexanes. The layers were separated and the organic phase was washed with water $(1 \times 2 \mathrm{~L})$ and saturated aqueous sodium chloride $(1 \times 1 \mathrm{~L})$, dried over anhydrous sodium sulfate, filtered and concentrated to provide (5S,7S,8R / 5R,7R,8S)-8-((tertbutyldiphenylsilyl)oxy)-7-methyl-7-vinyl-1-oxa-3-azaspiro[4.5]decan2-one racemate (29 g, 58 mmol, $79 \%$ yield) as a light yellow. LC-MS $\mathrm{C}_{27} \mathrm{H}_{35} \mathrm{NO}_{3} \mathrm{SiNa}[\mathrm{M}+\mathrm{Na}$ ] calculated: 472.2 , observed: 472.2, $11.77 \mathrm{~min}$ (ret. time); ${ }^{1} \mathrm{H}$ NMR (400 MHz, DMSO- $\left.d_{6}\right) \delta$ ppm 0.97 - 1.00 (m, 9 H) 1.22 - 1.24 $(\mathrm{m}, 3 \mathrm{H}) 1.31-1.40(\mathrm{~m}, 2 \mathrm{H}) 1.51-1.58(\mathrm{~m}, 1 \mathrm{H}) 1.63-1.76(\mathrm{~m}, 3 \mathrm{H}) 2.98-3.13(\mathrm{~m}, 2 \mathrm{H}) 3.59-$ $3.66(\mathrm{~m}, 1 \mathrm{H}) 4.95-5.04(\mathrm{~m}, 2 \mathrm{H}) 5.79-5.88(\mathrm{~m}, 1 \mathrm{H}) 7.39$ - $7.40(\mathrm{~m}, 2 \mathrm{H}) 7.45-7.46(\mathrm{~m}, 2 \mathrm{H})$ 7.64 - $7.66(\mathrm{~m}, 2 \mathrm{H}) 7.70-7.72(\mathrm{~m}, 4 \mathrm{H})$.

\section{(5S,7R,8R / 5R,7S,8S)-8-((tert-Butyldiphenylsilyl)oxy)-7-methyl-2-oxo-1-oxa-3-}

\section{azaspiro[4.5]decane-7-carbaldehyde (racemate, 14)}

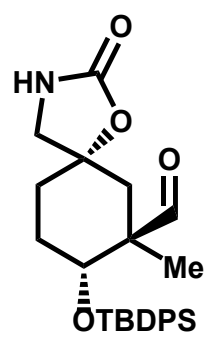

8-((tert-Butyldiphenylsilyl)oxy)-7-methyl-7-vinyl-1-oxa-3-azaspiro[4.5]decan-2-one racemate (40 $\mathrm{g}, 89 \mathrm{mmol})$ was dissolved in tetrahydrofuran (THF) $(1 \mathrm{~L})$ and treated with sodium bicarbonate (22.4 g, $267 \mathrm{mmol})$, sodium periodate $(57.1 \mathrm{~g}, 267 \mathrm{mmol})$, and water $(1 \mathrm{~L})$, followed by osmium tetroxide $(2.5 \mathrm{wt} \%$ in $t-\mathrm{BuOH}, 90 \mathrm{~mL}, 8.9 \mathrm{mmol})$ at room temperature. The reaction mixture was stirred for 2.5 hours, after which HPLC (Eclipse XDB-C18, 4.6 × 250 mm, 5 micron, $2.5 \mathrm{~mL} / \mathrm{min}$, gradient from 1-99\% $\mathrm{CH}_{3} \mathrm{CN}\left(0.1 \%\right.$ trifluoroacetic acid) / $\mathrm{H}_{2} \mathrm{O}(0.1 \%$ trifluoroacetic acid), 10 minutes plus 2 minute plateau at $99 \% \mathrm{CH}_{3} \mathrm{CN}(0.1 \%$ trifluoroacetic acid)) and LCMS (SunFire, C18, $5 \mu \mathrm{m}, 3.0 \times 50 \mathrm{~mm}$, gradient $10-100 \% \mathrm{CH}_{3} \mathrm{CN}$ in $\mathrm{H}_{2} \mathrm{O}$ with 0.1\% TFA, $2.5 \mathrm{~min}$ ) indicated that most of the starting material had been converted to the corresponding diastereomeric diols with a minor amount of aldehyde product. An additional sodium periodate $(57.1 \mathrm{~g}, 267 \mathrm{mmol})$ aliquot was added and the reaction mixture was stirred for an additional hour, after which time the reaction was complete. The reaction mixture was quenched with 300 grams of sodium thiosulfate and $300 \mathrm{~mL}$ of water and stirred for 30 minutes. The mixture was then poured into $2 \mathrm{~L}$ of water and $2 \mathrm{~L}$ of ethyl acetate. The layers were separated and the organic phase was washed with 
water ( $3 \times 2 \mathrm{~L})$, dried over anhydrous sodium sulfate, filtered and concentrated to give a light yellow oil. The crude material was adsorbed onto $\sim 200 \mathrm{~g}$ of $\mathrm{SiO}_{2}$ and purified by silica gel column chromatography (330 $\mathrm{g} \mathrm{SiO}_{2}$, gradient elution from 25\% EtOAc in hexanes to $100 \% \mathrm{EtOAc}$ ) to provide 8-((tert-butyldiphenylsilyl)oxy)-7-methyl-2-oxo-1-oxa-3-azaspiro[4.5]decane-7carbaldehyde racemate $(42 \mathrm{~g}, 88 \mathrm{mmol}, 99 \%$ yield).

$(5 S, 7 S, 8 R$ / $5 R, 7 R, 8 S)-7-(($ Allylamino)methyl)-8-((tert-butyldiphenylsilyl)oxy)-7-methyl-1oxa-3-azaspiro[4.5]decan-2-one (racemate)

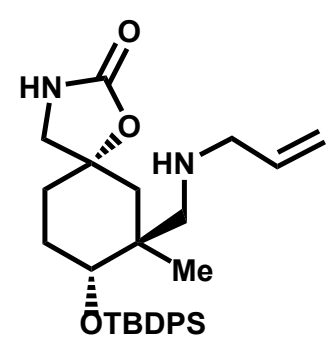

8-((tert-Butyldiphenylsilyl)oxy)-7-methyl-2-oxo-1-oxa-3-azaspiro[4-5]decane-7-carbaldehyde racemate $(36 \mathrm{~g}, 80 \mathrm{mmol})$ was dissolved in 1,2-dichloroethane (DCE) $(400 \mathrm{~mL})$ and treated with prop-2-en-1-amine $(41.8 \mathrm{~mL}, 558 \mathrm{mmol})$ and sodium triacetoxyborohydride $(42.2 \mathrm{~g}, 199 \mathrm{mmol})$ under an atmosphere of nitrogen. The reaction was stirred for $18 \mathrm{~h}$, after which time HPLC (Eclipse XDB-C18, $4.6 \times 250 \mathrm{~mm}, 5$ micron, $2.5 \mathrm{~mL} / \mathrm{min}$, gradient from 1-99\% $\mathrm{CH}_{3} \mathrm{CN}(0.1 \%$ trifluoroacetic acid) / $\mathrm{H}_{2} \mathrm{O}$ (0.1\% trifluoroacetic acid), 10 minutes plus 2 minute plateau at $99 \%$ $\mathrm{CH}_{3} \mathrm{CN}(0.1 \%$ trifluoroacetic acid) and LCMS (SunFire, C18, $5 \mu \mathrm{m}, 3.0 \times 50 \mathrm{~mm}$, gradient 10 $100 \% \mathrm{CH}_{3} \mathrm{CN}$ in $\mathrm{H}_{2} \mathrm{O}$ with $0.1 \% \mathrm{TFA}, 2.5 \mathrm{~min}$ ) indicated that the reaction was only partially complete. The reaction mixture was treated with an additional portions of prop-2-en-1-amine (41.8 $\mathrm{mL}, 558 \mathrm{mmol}$ ) and sodium triacetoxyborohydride $(42.2 \mathrm{~g}, 199 \mathrm{mmol})$ and allowed to stir for an additional $42 \mathrm{~h}$ to reach completion. The reaction mixture was treated with $400 \mathrm{~mL}$ of halfsaturated sodium bicarbonate and stirred for 20 minutes. The mixture was partitioned with ethyl acetate $(400 \mathrm{~mL})$ and water $(200 \mathrm{~mL})$ and the layers were separated. The organic layer was washed with water $(1 \times 500 \mathrm{~mL}), 10 \%$ citric acid solution $(1 \times 500 \mathrm{~mL})$, and saturated aqueous sodium bicarbonate $(1 \times 500 \mathrm{~mL})$. The organics were dried over anhydrous sodium sulfate, filtered and concentrated to give a brown oil $(41 \mathrm{~g})$ which was used in the next step without further purification. LC-MS $\mathrm{C}_{29} \mathrm{H}_{41} \mathrm{~N}_{2} \mathrm{O}_{3} \mathrm{Si}[\mathrm{M}+\mathrm{H}]$ calculated: 493.3, observed: 493.2, 1.32 min (ret. time); ${ }^{1} \mathrm{H}$ NMR $\left(400 \mathrm{MHz}\right.$, DMSO- $\left.d_{6}\right) \delta \mathrm{ppm} 0.99(\mathrm{~s}, 9 \mathrm{H}) 1.07-1.10(\mathrm{~m}, 3 \mathrm{H}) 1.54-1.75(\mathrm{~m}, 6 \mathrm{H}) 2.26$ - $2.33(\mathrm{~m}, 1 \mathrm{H}) 2.44-2.49(\mathrm{~m}, 1 \mathrm{H}) 3.05(\mathrm{br} \mathrm{s}, 1 \mathrm{H}) 3.18-3.21(\mathrm{~m}, 1 \mathrm{H}) 3.68-3.70(\mathrm{~m}, 2 \mathrm{H}) 3.71$ - $3.78(\mathrm{~m}, 1 \mathrm{H}) 4.43-4.64(\mathrm{~m}, 1 \mathrm{H}) 5.12(\mathrm{dd}, \mathrm{J}=17.32,1.76 \mathrm{~Hz}, 2 \mathrm{H}) 5.80-5.84(\mathrm{~m}, 1 \mathrm{H}) 7.44$ $7.54(\mathrm{~m}, 6 \mathrm{H}) 7.64-7.71(\mathrm{~m}, 4 \mathrm{H})$. 
(5S,7S,8R / 5R,7R,8S)-7-(Aminomethyl)-8-((tert-butyldiphenylsilyl)oxy)-7-methyl-1-oxa-3azaspiro[4.5]decan-2-one (racemate, 15)

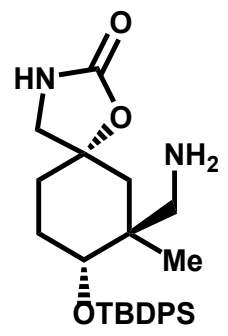

7-((Allylamino)methyl)-8-((tert-butyldiphenylsilyl)oxy)-7-methyl-1-oxa-3-azaspiro[4.5]decan-2one racemate $(40 \mathrm{~g}, 81 \mathrm{mmol})$ was dissolved in ethanol $(500 \mathrm{~mL})$ and treated with tetrakis(triphenylphosphine)palladium(0) (7.2 g, $6.23 \mathrm{mmol})$ and 1,3-dimethylpyrimidine2,4,6(1H,3H,5H)-trione $(15.2 \mathrm{~g}, 97 \mathrm{mmol})$ under an atmosphere of nitrogen. The reaction was stirred at room temperature for $18 \mathrm{~h}$, after which HPLC (Eclipse XDB-C18, 4.6 × $250 \mathrm{~mm}, 5$ micron, $2.5 \mathrm{~mL} / \mathrm{min}$, gradient from $1-99 \% \mathrm{CH}_{3} \mathrm{CN}\left(0.1 \%\right.$ trifluoroacetic acid) $/ \mathrm{H}_{2} \mathrm{O}(0.1 \%$ trifluoroacetic acid), 10 minutes plus 2 minute plateau at $99 \% \mathrm{CH}_{3} \mathrm{CN}(0.1 \%$ trifluoroacetic acid)) and LCMS ((SunFire, C18, $5 \mu \mathrm{m}, 3.0 \times 50 \mathrm{~mm}$, gradient $10-100 \% \mathrm{CH}_{3} \mathrm{CN}$ in $\mathrm{H}_{2} \mathrm{O}$ with $0.1 \%$ TFA, $2.5 \mathrm{~min}$ ) indicated that the reaction was complete. The solvent was removed under reduced pressure. The thick, sticky yellow residue was suspended in acetonitrile $(500 \mathrm{~mL})$ and triturated for 10 minutes, but the residue remained sticky and taffy-like. The acetonitrile and residual ethanol were removed under reduced pressure. Attempts were made to triturate the residue with acetonitrile, ether, toluene, and ethyl acetate, but none were successful likely due to the barbituric acid byproduct interfering with precipitation. The residue was dissolved in $1 \mathrm{~L}$ of ethyl acetate and washed twice with $1 \mathrm{~L}$ of a mixture of $1 \mathrm{~N} \mathrm{NaOH}$ and saturated aqueous sodium chloride in a 1:1 ratio in order to remove the barbituric acid byproduct. The organic layer was dried over anhydrous sodium sulfate and quickly filtered, then concentrated to give a thick black / brown residue. The crude material $(36 \mathrm{~g})$ was carried forward as a 7:1 mixture of racemic diastereomers. LC-MS $\mathrm{C}_{26} \mathrm{H}_{37} \mathrm{~N}_{2} \mathrm{O}_{3} \mathrm{Si}[\mathrm{M}+\mathrm{H}]$ calculated: 453.3, observed: 453.2, 1.24 min (ret. time). 
3-((((5S,7S,8R / 5R,7R,8S)-8-((tert-Butyldiphenylsilyl)oxy)-7-methyl-2-oxo-1-oxa-3azaspiro[4.5]decan-7-yl)methyl)amino)-4-nitrobenzonitrile (racemate, 16)

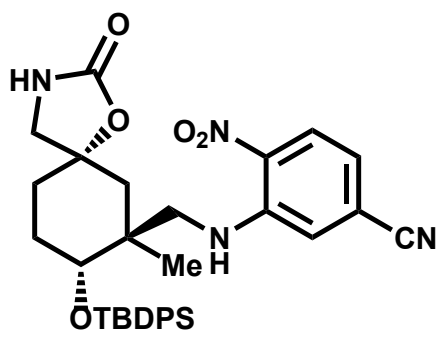

7-(Aminomethyl)-8-((tert-butyldiphenylsilyl)oxy)-7-methyl-1-oxa-3-azaspiro[4.5]decan-2-one racemate $(1.5 \mathrm{~g}, 3.31 \mathrm{mmol})$ was dissolved in acetonitrile and treated with 3-fluoro-4nitrobenzonitrile $(661 \mathrm{mg}, 4.0 \mathrm{mmol})$ and potassium carbonate $(916 \mathrm{mg}, 6.6 \mathrm{mmol})$ under an atmosphere of nitrogen. The reaction was stirred at $50{ }^{\circ} \mathrm{C}$ for 60 minutes, after which time HPLC (Eclipse XDB-C18, 4.6 × $250 \mathrm{~mm}, 5$ micron, $2.5 \mathrm{~mL} / \mathrm{min}$, gradient from $1-99 \% \mathrm{CH}_{3} \mathrm{CN}(0.1$ \%trifluoroacetic acid) / $\mathrm{H}_{2} \mathrm{O}$ (0.1\% trifluoroacetic acid), 10 minutes plus 2 minute plateau at $99 \%$ $\mathrm{CH}_{3} \mathrm{CN}(0.1 \%$ trifluoroacetic acid)) and LCMS (SunFire, C18, $5 \mu \mathrm{m}, 3.0 \times 50 \mathrm{~mm}$, gradient 10 $100 \% \mathrm{CH}_{3} \mathrm{CN}$ in $\mathrm{H}_{2} \mathrm{O}$ with $0.1 \%$ TFA, $2.5 \mathrm{~min}$ ) indicated that the reaction was complete. The solvent was removed under reduced pressure and the residue was partitioned with ethyl acetate $(500 \mathrm{~mL})$ and water $(500 \mathrm{~mL})$. The layers were separated and the organic phase was washed with water $(1 \times 500 \mathrm{~mL})$ and saturated aqueous sodium chloride $(1 \times 500 \mathrm{~mL})$, dried over sodium sulfate, filtered, and concentrated to give a dark red oil. The crude material was absorbed onto $\sim 200 \mathrm{~g}$ of silica gel and purified by silica gel column chromatography $\left(750 \mathrm{~g} \mathrm{SiO}_{2}\right.$, gradient elution from $0 \%$ EtOAc in hexanes to $50 \%$ EtOAc in hexanes over one hour, 20 minute plateau at 50 / $50 \mathrm{EtOAc} /$ hexanes, then gradient from 50 / 50 to 100\% EtOAc in hexanes over 30 minutes) to give 3-(((8-((tert-butyldiphenylsilyl)oxy)-7-methyl-2-oxo-1-oxa-3-azaspiro[4.5]decan-7$\mathrm{yl}$ )methyl)amino)-4-nitrobenzonitrile racemate $(1.7 \mathrm{~g}, 2.70 \mathrm{mmol}, 81 \%$ yield) as a light orange foam. LC-MS $\mathrm{C}_{33} \mathrm{H}_{39} \mathrm{~N}_{4} \mathrm{O}_{5} \mathrm{Si}\left[\mathrm{M}+\mathrm{H}\right.$ ] calculated: 599.3, observed: 599.3, 2.09 min (ret. time). ${ }^{1} \mathrm{H}$ NMR (400 MHz, DMSO- $\left.d_{6}\right) \delta$ ppm $0.99(\mathrm{~s}, 9 \mathrm{H}) 1.21$ - $1.27(\mathrm{~m}, 3 \mathrm{H}) 1.32$ - $1.38(\mathrm{~m}, 1 \mathrm{H}) 1.47$ $1.55(\mathrm{~m}, 1 \mathrm{H}) 1.57$ - $1.62(\mathrm{~m}, 1 \mathrm{H}) 1.66-1.80(\mathrm{~m}, 2 \mathrm{H}) 1.81$ - $1.89(\mathrm{~m}, 1 \mathrm{H}) 2.99-3.08(\mathrm{~m}, 1 \mathrm{H})$ 3.16 - $3.23(\mathrm{~m}, 1 \mathrm{H}) 3.37$ - $3.41(\mathrm{~m}, 1 \mathrm{H}) 3.76$ - $3.86(\mathrm{~m}, 1 \mathrm{H}) 4.41$ - $4.57(\mathrm{~m}, 1 \mathrm{H}) 7.03$ - $7.09(\mathrm{~m}$, $1 \mathrm{H}) 7.34$ - $7.40(\mathrm{~m}, 2 \mathrm{H}) 7.42$ - $7.50(\mathrm{~m}, 4 \mathrm{H}) 7.58$ - $7.62(\mathrm{~m}, 2 \mathrm{H}) 7.63$ - $7.68(\mathrm{~m}, 2 \mathrm{H}) 7.97$ - 8.04 $(\mathrm{m}, 1 \mathrm{H}) 8.18-8.25(\mathrm{~m}, 1 \mathrm{H})$.

\section{4-Amino-3-((((5S,7S,8R / 5R,7R,8S)-8-((tert-butyldiphenylsilyl)oxy)-7-methyl-2-oxo-1-oxa-3- azaspiro[4.5]decan-7-yl)methyl)amino)benzonitrile (racemate)}




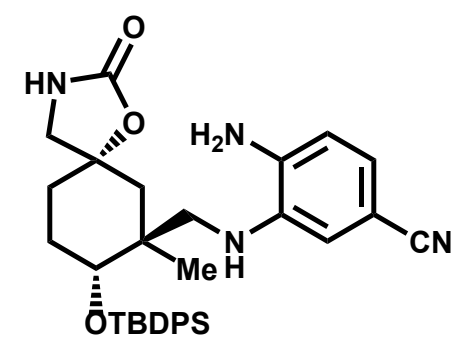

3-(((8-((tert-Butyldiphenylsilyl)oxy)-7-methyl-2-oxo-1-oxa-3-azaspiro[4-5]decan-7-

$\mathrm{yl}$ )methyl)amino)-4-nitrobenzonitrile racemate (15.5 g, $25.9 \mathrm{mmol})$ was dissolved in ethyl acetate $(100 \mathrm{~mL})$ and added to a nitrogen-charged three-necked flask containing $10 \%$ palladium on carbon $(13.8 \mathrm{~g}, 13.0 \mathrm{mmol})$. Methanol $(100 \mathrm{~mL})$ was added as a co-solvent. The flask was flushed with hydrogen gas and then placed under $1 \mathrm{~atm}$ of hydrogen pressure (balloon) for 90 minutes, after which HPLC (Eclipse XDB-C18, 4.6 x 250 mm, 5 micron, 2.5 mL/min, gradient from 1-99\% $\mathrm{CH}_{3} \mathrm{CN}\left(0.1 \%\right.$ trifluoroacetic acid) $/ \mathrm{H}_{2} \mathrm{O}(0.1 \%$ trifluoroacetic acid $), 10$ minutes plus 2 minute plateau at $99 \% \mathrm{CH}_{3} \mathrm{CN}(0.1 \%$ trifluoroacetic acid) and LCMS (SunFire, C18, $5 \mu \mathrm{m}, 3.0 \times 50 \mathrm{~mm}$, gradient $10-100 \% \mathrm{CH}_{3} \mathrm{CN}$ in $\mathrm{H}_{2} \mathrm{O}$ with $0.1 \%$ TFA, $2.5 \mathrm{~min}$ ) indicated that the reaction was complete. The reaction mixture was diluted with a 1:1:1 mixture of dichloromethane, ethyl acetate and ethanol and 100 grams of celite. The reaction mixture was filtered and the filter cake was rinsed with the aformentioned solvent mixture. The solvent was removed under reduced pressure to provide 4-amino-3-(((5S,7S,8R)-8-((tert-butyldiphenylsilyl)oxy)-7-methyl-2-oxo-1-oxa-3azaspiro[4.5]decan-7-yl)methyl)amino)benzonitrile racemate as a light green powder (14.7 g, $23.3 \mathrm{mmol}, 90 \%$ yield) which was carried immediately into the next reaction due to rapid air oxidation of the ortho phenylenediamine. LC-MS $\mathrm{C}_{33} \mathrm{H}_{41} \mathrm{~N}_{4} \mathrm{O}_{3} \mathrm{Si}[\mathrm{M}+\mathrm{H}]$ calculated: 569.3, observed: 569.2, $1.90 \mathrm{~min}$ (ret. time).

\section{1-((5S,7S,8R ～/ 5R,7R,8S)-8-((tert-Butyldiphenylsilyl)oxy)-7-methyl-2-oxo-1-oxa-3-} azaspiro[4.5]decan-7-yl)methyl)-1H-benzo[d]imidazole-6-carbonitrile (racemate, 17)

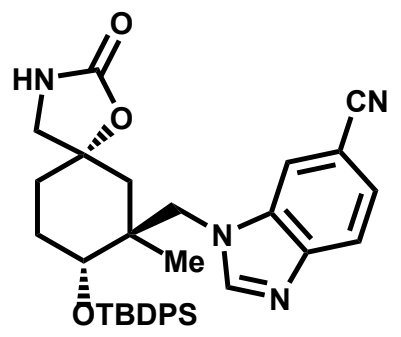

4-Amino-3-(((8-((tert-butyldiphenylsilyl)oxy)-7-methyl-2-oxo-1-oxa-3-azaspiro[4.5]decan-7yl)methyl)amino)-4-benzonitrile racemate $(1.5 \mathrm{~g}, 2.7 \mathrm{mmol})$ was dissolved in trimethyl orthoformate $(18 \mathrm{~mL}, 162 \mathrm{mmol})$ and treated with formic acid (2 mL, $54.2 \mathrm{mmol}$ ) in a nitrogen- 
charged flask. The reaction mixture was stirred at room temperature for $18 \mathrm{~h}$ after which HPLC (Eclipse XDB-C18, $4.6 \times 250 \mathrm{~mm}, 5$ micron, $2.5 \mathrm{~mL} / \mathrm{min}$, gradient from 1-99\% $\mathrm{CH}_{3} \mathrm{CN}(0.1 \%$ trifluoroacetic acid) / $\mathrm{H}_{2} \mathrm{O}$ (0.1\% trifluoroacetic acid), 10 minutes plus 2 minute plateau at $99 \%$ $\mathrm{CH}_{3} \mathrm{CN}(0.1 \%$ trifluoroacetic acid)) and LCMS ((SunFire, C18, 5um, $3.0 \times 50 \mathrm{~mm}$, gradient $10-$ $100 \% \mathrm{CH}_{3} \mathrm{CN}$ in $\mathrm{H}_{2} \mathrm{O}$ with $0.1 \% \mathrm{TFA}, 2.5 \mathrm{~min}$ ) indicated that the reaction was complete. The volatiles were removed under reduced pressure and the residue was partitioned with $200 \mathrm{~mL}$ of ethyl acetate and $200 \mathrm{~mL}$ of water. The organic layer was separated, washed with water (1 x 200 $\mathrm{mL}$ ) and saturated aqueous sodium chloride $(1 \times 200 \mathrm{~mL})$, dried over anhydrous sodium sulfate, filtered, and concentrated to give 1-(((5S,7S,8R)-8-((tert-butyldiphenylsilyl)oxy)-7-methyl-2-oxo1-oxa-3-azaspiro[4.5]decan-7-yl)methyl)-1 H-benzo[d]imidazole-6-carbonitrile racemate (1.5 g, $2.3 \mathrm{mmol}, 86 \%$ yield) as a light yellow oil which was taken on to the next step without further purification. LC-MS $\mathrm{C}_{34} \mathrm{H}_{39} \mathrm{~N}_{4} \mathrm{O}_{3} \mathrm{Si}[\mathrm{M}+\mathrm{H}]$ calculated: 579.3, observed: 579.2, 1.73 min (ret. time); ${ }^{1} \mathrm{H}$ NMR $\left(400 \mathrm{MHz}\right.$, DMSO-d $\left.d_{6}\right) \delta$ ppm 1.09 (s, $\left.9 \mathrm{H}\right) 1.20$ - $1.25(\mathrm{~m}, 3 \mathrm{H}) 1.32$ - $1.49(\mathrm{~m}, 2 \mathrm{H}) 1.50$ - $1.60(\mathrm{~m}, 1 \mathrm{H}) 1.62-1.78(\mathrm{~m}, 2 \mathrm{H}) 1.79-1.86(\mathrm{~m}, 1 \mathrm{H}) 2.87-3.02(\mathrm{~m}, 1 \mathrm{H}) 3.16-3.24(\mathrm{~m}, 1 \mathrm{H})$ 3.80 - $3.89(\mathrm{~m}, 1 \mathrm{H}) 4.21$ - $4.31(\mathrm{~m}, 1 \mathrm{H}) 4.36$ - $4.45(\mathrm{~m}, 1 \mathrm{H}) 7.32$ - $7.43(\mathrm{~m}, 1 \mathrm{H}) 7.46$ - $7.53(\mathrm{~m}$, 5 H) 7.58 - $7.63(\mathrm{~m}, 2 \mathrm{H}) 7.67$ - $7.73(\mathrm{~m}, 2 \mathrm{H}) 7.75$ - $7.79(\mathrm{~m}, 1 \mathrm{H}) 7.81$ - $7.87(\mathrm{~m}, 1 \mathrm{H}) 8.16$ - 8.26 $(\mathrm{m}, 1 \mathrm{H}) 8.27-8.32(\mathrm{~m}, 1 \mathrm{H})$.

1-((5S,7S,8R ～/ 5R,7R,8S)-8-((tert-Butyldiphenylsilyl)oxy)-3-(5-(2-hydroxypropan-2yl)pyrazin-2-yl)-7-methyl-2-oxo-1-oxa-3-azaspiro[4.5]decan-7-yl)methyl)-1Hbenzo[d]imidazole-6-carbonitrile (racemate)

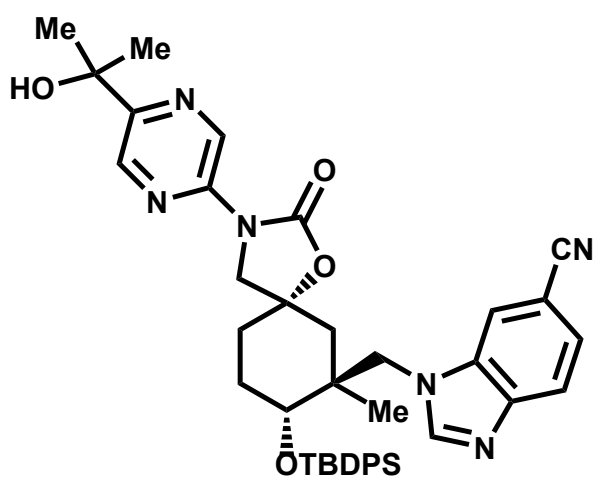

1-((8-((tert-butyldiphenylsilyl)oxy)-7-methyl-2-oxo-1-oxa-3-azaspiro[4.5]decan-7-yl)methyl)-1Hbenzo[d]imidazole-6-carbonitrile racemate $(1.5 \mathrm{~g}, 2.6 \mathrm{mmol})$ was dissolved in 1,4-dioxane $(20 \mathrm{~mL})$ and treated with copper(I) iodide (543 mg, $2.9 \mathrm{mmol}), \mathrm{N1}, \mathrm{N2}$-dimethylethane-1,2-diamine (0.614 $\mathrm{mL}, 5.70 \mathrm{mmol})$, and potassium phosphate tribasic $(1.2 \mathrm{~g}, 5.7 \mathrm{mmol})$ under an atmosphere of nitrogen at room temperature. The reaction mixture was heated to $100{ }^{\circ} \mathrm{C}$ and a solution of $2-(5-$ 
bromopyrazin-2-yl)propan-2-ol $(0.844 \mathrm{~g}, 3.89 \mathrm{mmol})$ was added and stirred at $100{ }^{\circ} \mathrm{C}$ for 80 minutes, after which HPLC (Eclipse XDB-C18, 4.6 × $250 \mathrm{~mm}, 5$ micron, $2.5 \mathrm{~mL} / \mathrm{min}$, gradient from 1-99\% $\mathrm{CH}_{3} \mathrm{CN}\left(0.1 \%\right.$ trifluoroacetic acid) $/ \mathrm{H}_{2} \mathrm{O}(0.1 \%$ trifluoroacetic acid), 10 minutes plus 2 minute plateau at $99 \% \mathrm{CH}_{3} \mathrm{CN}(0.1 \%$ trifluoroacetic acid) and LCMS (SunFire, C18, $5 \mu \mathrm{m}, 3.0 \mathrm{x}$ $50 \mathrm{~mm}$, gradient $10-100 \% \mathrm{CH}_{3} \mathrm{CN}$ in $\mathrm{H}_{2} \mathrm{O}$ with $0.1 \%$ TFA, $2.5 \mathrm{~min}$ ) indicated that the reaction was complete. The reaction mixture was allowed to cool to room temperature and then poured into $500 \mathrm{~mL}$ of $10 \%$ aqueous ammonium hydroxide and $500 \mathrm{~mL}$ of ethyl acetate. The layers were separated and the organic layer was washed with $10 \%$ aqueous ammonium hydroxide $(1 \times 500$ $\mathrm{mL}$ ) and saturated sodium chloride $(1 \times 500 \mathrm{~mL})$, dried over anhydrous sodium sulfate, filtered, and concentrated to give a brown oil / foam. The crude material was purified by silica gel column chromatography (220 g silica gel dry load, gradient elution from 0 to $50 \%$ EtOAc in hexanes over 40 minutes, plateau at 50 / 50 EtOAc / hexanes for 10 minutes, then gradient elution from 50 to $100 \%$ EtOAc over 20 minutes $)$ to provide 1-((8-((tert-butyldiphenylsilyl)oxy)-3-(5-(2hydroxypropan-2-yl)pyrazin-2-yl)-7-methyl-2-oxo-1-oxa-3-azaspiro[4.5]decan-7-yl)methyl)-1Hbenzo[d]imidazole-6-carbonitrile racemate $(0.730 \mathrm{~g}, 0.919 \mathrm{mmol}, 36 \%$ yield) as a light yellow foam. LC-MS $\mathrm{C}_{41} \mathrm{H}_{45} \mathrm{~N}_{6} \mathrm{O}_{3} \mathrm{Si}\left[\mathrm{M}-\mathrm{OH}\right.$ ] calculated: 697.3, observed: 697.2, 1.94 min (ret. time); ${ }^{1} \mathrm{H}$ NMR (400 MHz, DMSO- $\left.d_{6}\right) \delta$ ppm $1.10(\mathrm{~s}, 9 \mathrm{H}) 1.23$ - $1.29(\mathrm{~m}, 3 \mathrm{H}) 1.32$ - $1.39(\mathrm{~m}, 1 \mathrm{H}) 1.39$ $1.42(\mathrm{~m}, 6 \mathrm{H}) 1.44-1.50(\mathrm{~m}, 1 \mathrm{H}) 1.68-1.78(\mathrm{~m}, 2 \mathrm{H}) 1.79-1.89(\mathrm{~m}, 2 \mathrm{H}) 3.58-3.65(\mathrm{~m}, 1 \mathrm{H})$ 3.68 - $3.74(\mathrm{~m}, 1 \mathrm{H}) 3.87$ - $3.95(\mathrm{~m}, 1 \mathrm{H}) 4.22$ - $4.33(\mathrm{~m}, 1 \mathrm{H}) 4.37$ - $4.50(\mathrm{~m}, 1 \mathrm{H}) 7.46$ - $7.56(\mathrm{~m}$, 7 H) 7.57 - $7.61(\mathrm{~m}, 1 \mathrm{H}) 7.69$ - $7.74(\mathrm{~m}, 2 \mathrm{H}) 7.75$ - $7.80(\mathrm{~m}, 2 \mathrm{H}) 7.81$ - $7.85(\mathrm{~m}, 1 \mathrm{H}) 8.19$ - 8.23 $(\mathrm{m}, 1 \mathrm{H}) 8.31-8.35(\mathrm{~m}, 1 \mathrm{H}) 8.51-8.54(\mathrm{~m}, 1 \mathrm{H})$. 
1-(((5S,7S,8R)-8-Hydroxy-3-(5-(2-hydroxypropan-2-yl)pyrazin-2-yl)-7-methyl-2-oxo-1-oxa3-azaspiro[4.5]decan-7-yl)methyl)-1H-benzo[d]imidazole-6-carbonitrile (1) 1-(((5R,7R,8S)-8-Hydroxy-3-(5-(2-hydroxypropan-2-yl)pyrazin-2-yl)-7-methyl-2-oxo-1-oxa3-azaspiro[4.5]decan-7-yl)methyl)-1H-benzo[d]imidazole-6-carbonitrile ((ent)-1)

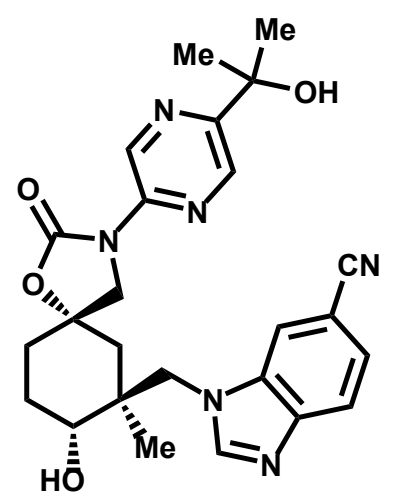

1

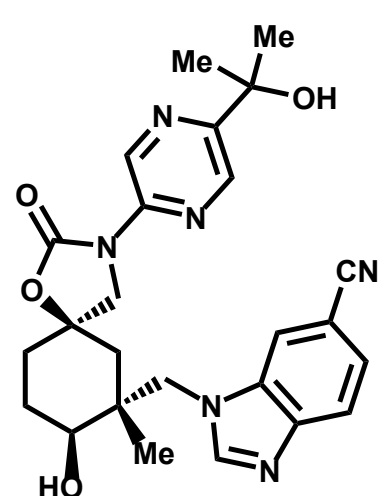

$(e n t)-1$

1-((-8-((tert-Butyldiphenylsilyl)oxy)-3-(5-(2-hydroxypropan-2-yl)pyrazin-2-yl)-7-methyl-2-oxo-1oxa-3-azaspiro[4.5]decan-7-yl)methyl)-1 $H$-benzo[d]imidazole-6-carbonitrile racemate $(5.2 \mathrm{~g}, 7.27$ $\mathrm{mmol}$ ) was dissolved in dimethyl sulfoxide (DMSO) and treated with tetramethylammonium fluoride $(2.0 \mathrm{~g}, 21.8 \mathrm{mmol})$ at room temperature. The reaction mixture was stirred for 2 hours, after which HPLC (Eclipse XDB-C18, 4.6 × $250 \mathrm{~mm}, 5$ micron, $2.5 \mathrm{~mL} / \mathrm{min}$, gradient from 1-99\% $\mathrm{CH}_{3} \mathrm{CN}\left(0.1 \%\right.$ trifluoroacetic acid) / $\mathrm{H}_{2} \mathrm{O}(0.1 \%$ trifluoroacetic acid $), 10$ minutes plus 2 minute plateau at $99 \% \mathrm{CH}_{3} \mathrm{CN}(0.1 \%$ trifluoroacetic acid)) and LCMS (SunFire, C18, $5 \mu \mathrm{m}, 3.0 \times 50 \mathrm{~mm}$, gradient $10-100 \% \mathrm{CH}_{3} \mathrm{CN}$ in $\mathrm{H}_{2} \mathrm{O}$ with $0.1 \% \mathrm{TFA}, 2.5 \mathrm{~min}$ ) indicated that the reaction was complete. The reaction mixture was poured slowly into a rapidly stirring mixture of $500 \mathrm{~mL}$ of water, $250 \mathrm{~mL}$ of saturated aqueous sodium chloride, and $250 \mathrm{~mL}$ of saturated aqueous sodium bicarbonate. A precipitate formed immediately, and the mixture was stirred for 20 minutes. The mixture was filtered and rinsed with $500 \mathrm{~mL}$ of water. The tan solid was air-dried for $18 \mathrm{~h}$ and subsequently dissolved in 10\% MeOH/DCM and dried over anhydrous sodium sulfate, filtered, and concentrated to give a tan solid that was triturated with $500 \mathrm{~mL}$ of hexanes. The compound mixture was absorbed onto $\sim 100 \mathrm{~g}$ of silica gel and purified by silica gel column chromatography (200 g silica gel dry-load, isocratic elution with $10 \% \mathrm{MeOH} / \mathrm{DCM}$ ) to provide a racemic mixture of 1 and (ent)-1 (2.2 g, $4.52 \mathrm{mmol}, 62 \%$ yield). The separated enantiomers were isolated by preparative HPLC (Chiralcel OD-H ( $5 \mu, 30 \times 250 \mathrm{~mm}, \mathrm{MeOH}, 45 \mathrm{~mL} / \mathrm{min}$ flow rate) to afford:

1: $765 \mathrm{mg}$ (off-white solid, $1.59 \mathrm{mmol}, 22 \%$ yield); $100 \%$ ee by chiral analytical HPLC; $[\alpha]_{D}=+$ $24^{\circ}(\mathrm{c}=0.1, \mathrm{MeOH})$; 
(ent)-1: $734 \mathrm{mg}$ (off-white solid, $1.52 \mathrm{mmol}, 21 \%$ yield); $97.3 \%$ ee by chiral analytical HPLC.

\section{Full characterization of 1 by 2D NMR and VCD}

\begin{tabular}{|c|c|c|c|c|c|c|c|c|}
\hline Atom\# & C Shift & H Shift & H Multiplicity & HVolume & $\cos Y$ & NOESY & HHMBC & CHMBC \\
\hline 10 & 16.163 & 1.285 & $\mathrm{~s}$ & 3.295 & & & $1.62,3.45,4.20,4.44$ & $52.76,43.12,41.05,70.18$ \\
\hline 12 & 26.158 & 2.001 & $\mathrm{~m}$ & -0.932 & $1.80,3.45$ & & & \\
\hline 12 & 26.158 & 1.795 & $\mathrm{~m}$ & -1.012 & $3.45,2.00$ & & & \\
\hline 24,23 & 29.024 & 1.564 & $\mathrm{~d}(2.99)$ & 5.982 & & & & $156.65,71.92$ \\
\hline 13 & 34.583 & 2.064 & $\mathrm{~m}$ & -1.284 & 1.64 & 3.88 & $2.11,3.88$ & \\
\hline 13 & 34.583 & 1.638 & $\operatorname{brd}(4.10)$ & -1.166 & 2.06 & 3.45 & $2.11,3.88$ & \\
\hline 9 & 41.051 & & & & & & $1.28,1.62,2.11,3.45$ & \\
\hline 15 & 43.122 & 2.106 & $\mathrm{~m}$ & -0.964 & 1.62 & 3.88 & $1.28,3.88,4.20,4.44$ & $79.99,70.18,34.58,41.05$ \\
\hline 15 & 43.122 & 1.617 & $\mathrm{~m}$ & -1.173 & 2.11 & 3.88 & $1.28,3.88,4.20,4.44$ & $54.68,16.16,41.05$ \\
\hline 8 & 52.760 & 4.444 & $d(14.66)$ & -0.998 & 4.20 & 8.29 & $1.28,3.45$ & $148.45,43.12,135.01,16.16,70.18$ \\
\hline 8 & 52.760 & 4.196 & $d(14.82)$ & -0.999 & 4.44 & & $1.28,3.45$ & $148.45,135.01,43.12,70.18,16.16$ \\
\hline 16 & 54.679 & 3.881 & $\mathrm{~s}$ & -1.947 & & $2.06,1.62,2$ & 1.62 & $34.58,79.99,43.12,153.93$ \\
\hline 11 & 70.179 & 3.447 & $d d(11.43,4.18)$ & 1.028 & $1.80,2.00$ & 1.64 & $1.28,2.11,4.20,4.44$ & $52.76,41.05,16.16$ \\
\hline 22 & 71.923 & & & & & & 1.56 & \\
\hline 14 & 79.992 & & & & & & $2.11,3.88$ & \\
\hline 5 & 105.943 & & & & & & 7.84 & \\
\hline 6 & 116.256 & 8.286 & $d(0.79)$ & 1.007 & & 4.44 & 7.61 & $119.14,125.41,145.14,135.01$ \\
\hline 25 & 119.138 & & & & & & $7.61,8.29$ & \\
\hline 3 & 119.957 & 7.836 & $d(8.35)$ & 1.024 & 7.61 & & & $135.01,105.94$ \\
\hline 4 & 125.409 & 7.611 & $\mathrm{dd}(8.35,1.42)$ & 1.028 & 7.84 & & 8.29 & $116.26,119.14,145.14$ \\
\hline 19 & 133.471 & 9.305 & $d(1.42)$ & 1.015 & & & & $146.20,156.65$ \\
\hline 7 & 135.010 & & & & & & $4.20,4.44,7.84,8.29,8.41$ & \\
\hline 21 & 137.687 & 8.600 & $d(1.58)$ & 1.000 & & & & $156.65,146.20$ \\
\hline Atom\# & C Shift & H Shift & H Multiplicity & HVolume & $\cos Y$ & NOESY & H HMBC & $\mathrm{CHMBC}$ \\
\hline 2 & 145.141 & & & & & & $7.61,8.29,8.41$ & \\
\hline 18 & 146.195 & & & & & & $8.60,9.31$ & \\
\hline 1 & 148.447 & 8.414 & $\mathrm{~m}$ & 0.884 & & & $4.20,4.44$ & $135.01,145.14$ \\
\hline 17 & 153.931 & & & & & & 3.88 & \\
\hline \multirow[t]{2}{*}{20} & 156.645 & & & & & & $1.56,8.60,9.31$ & \\
\hline & & 1.689 & $\operatorname{brd}(3.15)$ & 0.047 & & & & \\
\hline
\end{tabular}



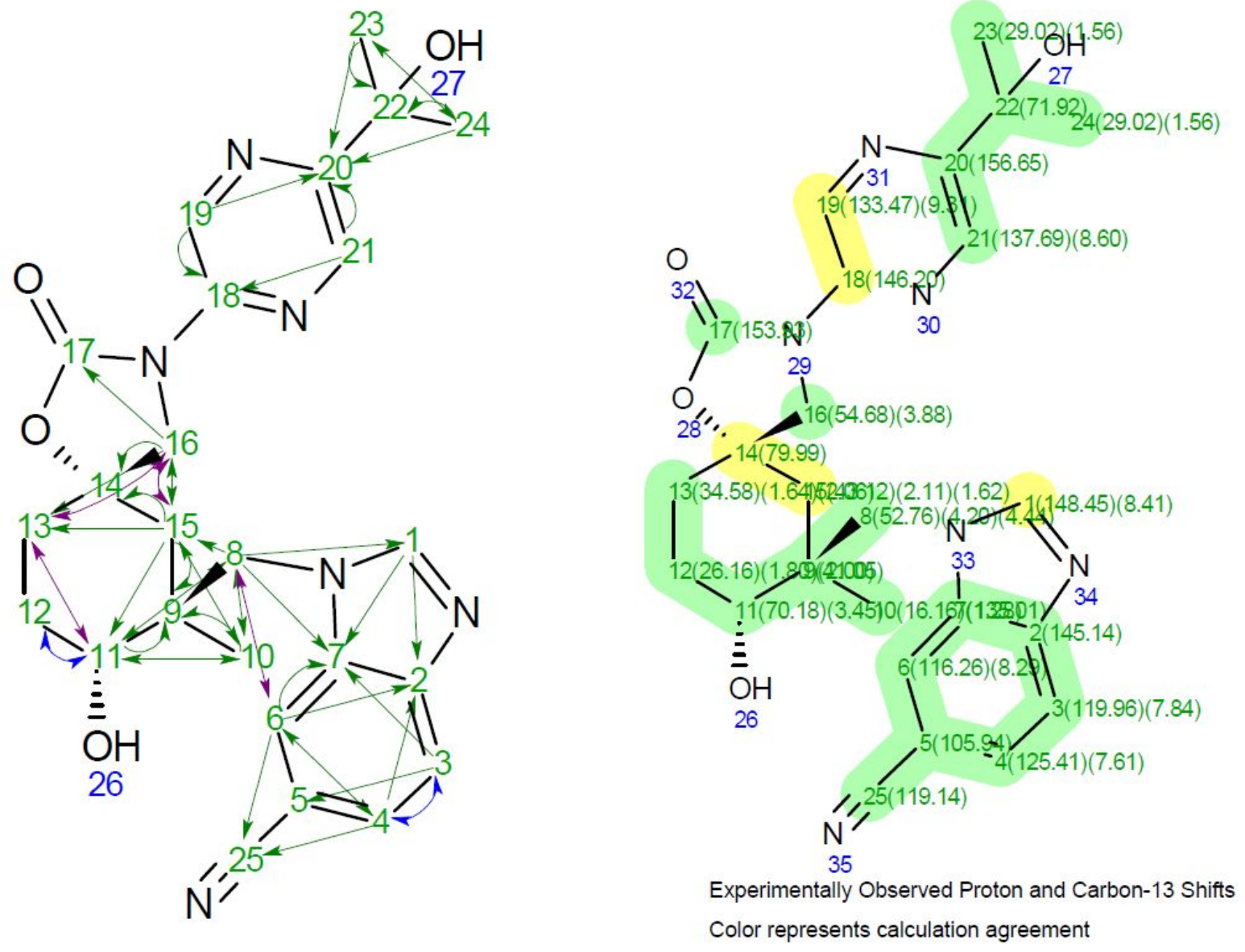

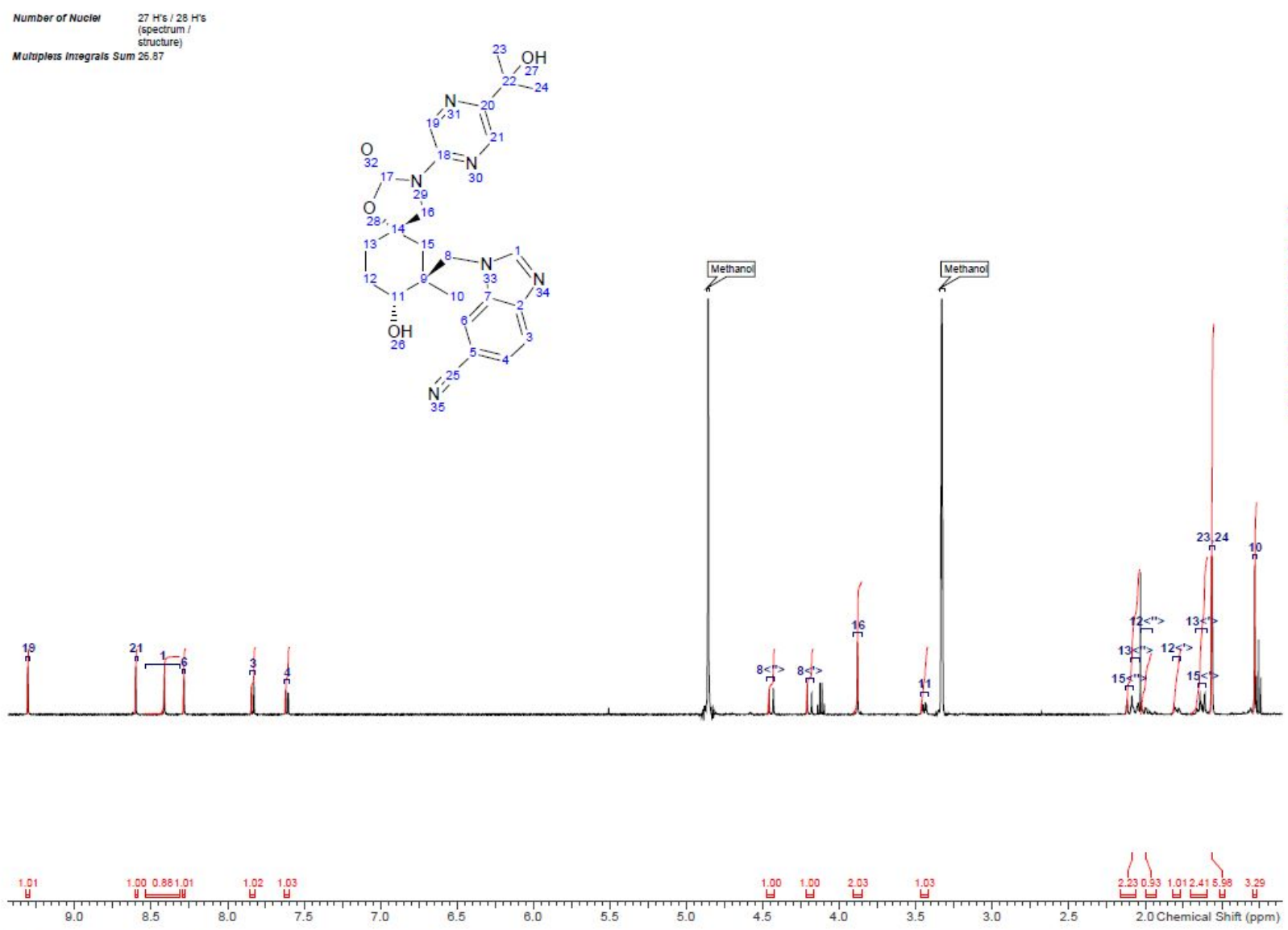

${ }^{1} \mathrm{H}$ NMR (METHANOL-d, $\left.500 \mathrm{MHz}\right): \delta(\mathrm{ppm}) 9.31(\mathrm{~d}, J=1.4 \mathrm{~Hz}, 1 \mathrm{H}), 8.60(\mathrm{~d}, J=1.6 \mathrm{~Hz}, 1 \mathrm{H}), 8.31-8.54(\mathrm{~m}, 1 \mathrm{H}), 8.29(\mathrm{~d}, J=0.8 \mathrm{~Hz}, 1 \mathrm{H}), 7.84(\mathrm{~d}, J=8.4 \mathrm{~Hz}, 1 \mathrm{H}), 7.61(\mathrm{dd}, J=8.4,1.4 \mathrm{~Hz}, 1 \mathrm{H}), 4.45$ $(\mathrm{d}, J=14.7 \mathrm{~Hz}, 1 \mathrm{H}), 4.20$ (d, $J=14.8 \mathrm{~Hz}, 1 \mathrm{H}), 3.88(\mathrm{~s}, 2 \mathrm{H}), 3.45$ (dd, $J=11.4,4.2 \mathrm{~Hz}, 1 \mathrm{H}), 2.08-2.13(\mathrm{~m}, 1 \mathrm{H}), 2.04-2.09(\mathrm{~m}, 1 \mathrm{H}), 1.96-2.03(\mathrm{~m}, 1 \mathrm{H}), 1.77-1.82(\mathrm{~m}, 1 \mathrm{H}), 1.69$ (br d, $J=3.2 \mathrm{~Hz}, 1 \mathrm{H})$, $1.60-1.65(\mathrm{~m}, 1 \mathrm{H}), 1.66(\mathrm{br} \mathrm{d}, J=4.1 \mathrm{~Hz}, 1 \mathrm{H}), 1.56(\mathrm{~d}, \mathrm{~J}=3.0 \mathrm{~Hz}, 6 \mathrm{H}), 1.28(\mathrm{~s}, 3 \mathrm{H})$ 

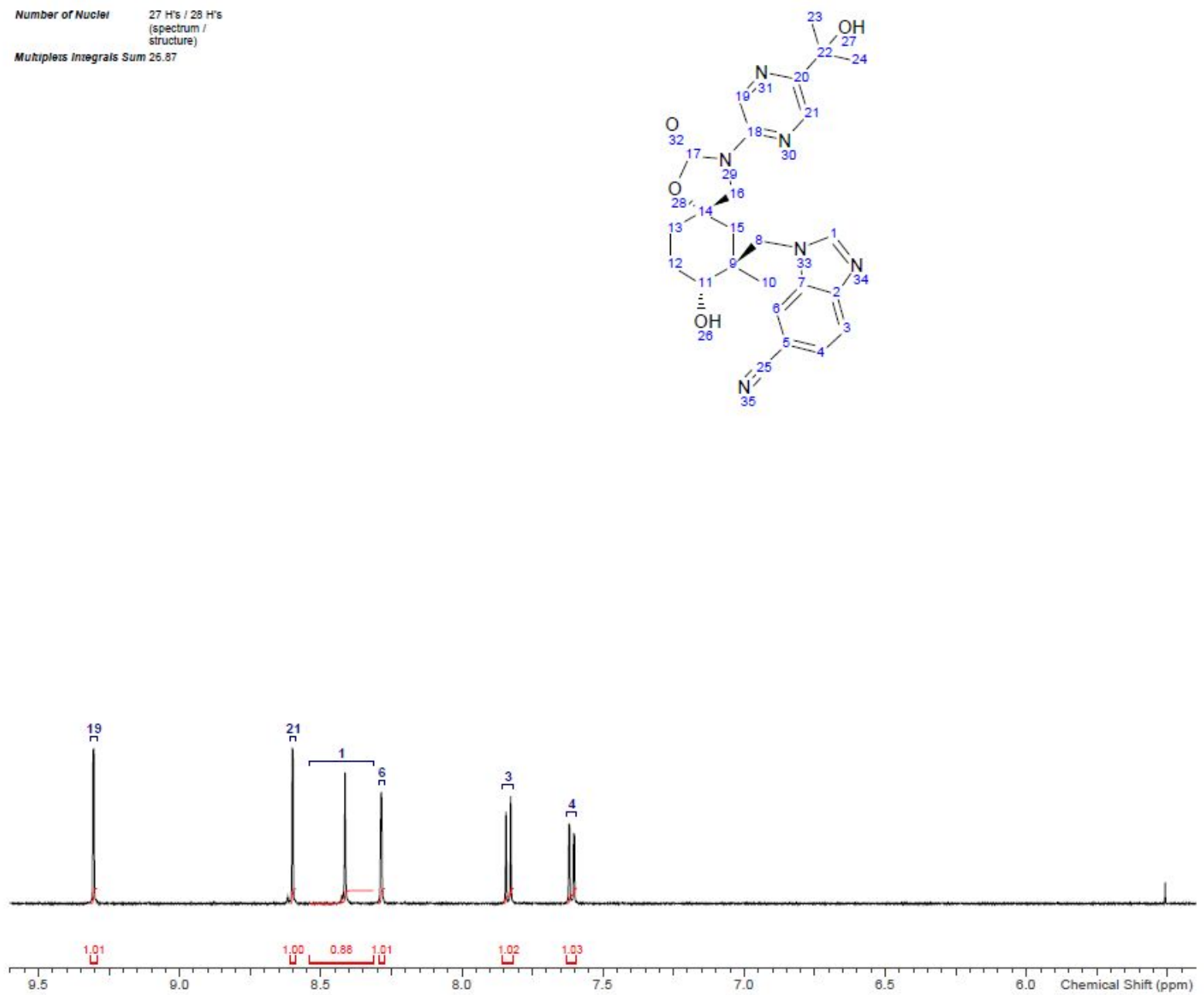

${ }^{1} \mathrm{H}$ NMR (METHANOL-d $\left.4,500 \mathrm{MHz}\right): \sigma(\mathrm{ppm}) 9.31(\mathrm{~d}, J=1.4 \mathrm{~Hz}, 1 \mathrm{H}), 8.60(\mathrm{~d}, J=1.6 \mathrm{~Hz}, 1 \mathrm{H}), 8.31-8.54(\mathrm{~m}, 1 \mathrm{H}), 8.29(\mathrm{~d}, J=0.8 \mathrm{~Hz}, 1 \mathrm{H}), 7.84(\mathrm{~d}, J=8.4 \mathrm{~Hz}, 1 \mathrm{H}), 7.61(\mathrm{dd}$, $J=8.4,1.4 \mathrm{~Hz}, 1 \mathrm{H}), 4.45(\mathrm{~d}, J=14.7 \mathrm{~Hz}, 1 \mathrm{H}), 4.20(\mathrm{~d}, J=14.8 \mathrm{~Hz}, 1 \mathrm{H}), 3.88(\mathrm{~s}, 2 \mathrm{H}), 3.45(\mathrm{dd}, J=11.4,4.2 \mathrm{~Hz}, 1 \mathrm{H}), 2.08-2.13(\mathrm{~m}, 1 \mathrm{H}), 2.04-2.09(\mathrm{~m}, 1 \mathrm{H}), 1.96-2.03(\mathrm{~m}, 1 \mathrm{H})$, $1.77-1.82(\mathrm{~m}, 1 \mathrm{H}), 1.69(\mathrm{br} \mathrm{d}, J=3.2 \mathrm{~Hz}, 1 \mathrm{H}), 1.60-1.65(\mathrm{~m}, 1 \mathrm{H}), 1.66(\mathrm{br} \mathrm{d}, J=4.1 \mathrm{~Hz}, 1 \mathrm{H}), 1.56(\mathrm{~d}, J=3.0 \mathrm{~Hz}, 6 \mathrm{H}), 1.28(\mathrm{~s}, 3 \mathrm{H})$ 


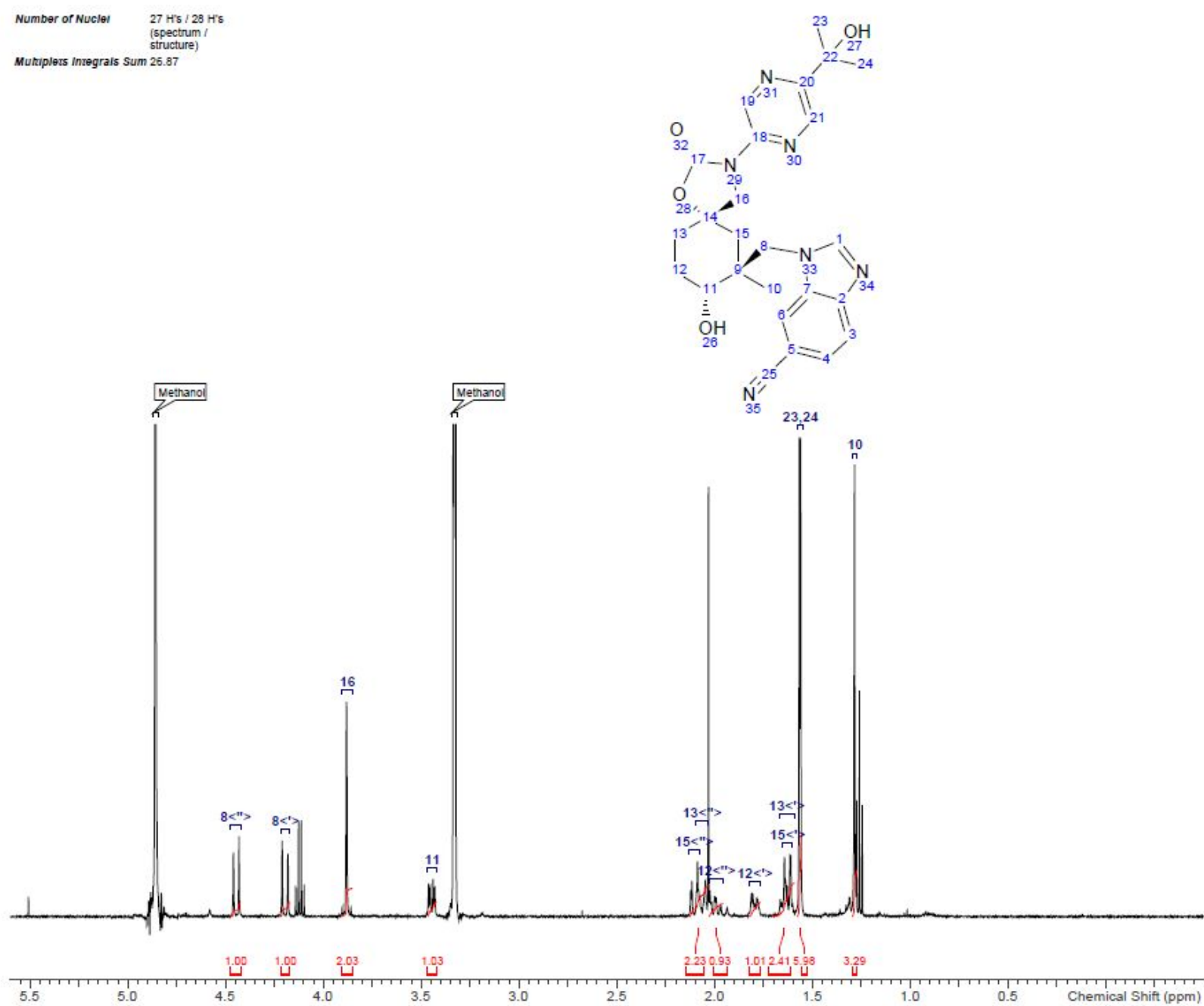

${ }^{1} \mathrm{H}$ NMR (METHANOL-d, $\left.500 \mathrm{MHz}\right): \delta(\mathrm{ppm}) 9.31(\mathrm{~d}, J=1.4 \mathrm{~Hz}, 1 \mathrm{H}), 8.60(\mathrm{~d}, J=1.6 \mathrm{~Hz}, 1 \mathrm{H}), 8.31-8.54(\mathrm{~m}, 1 \mathrm{H}), 8.29(\mathrm{~d}, J=0.8 \mathrm{~Hz}, 1 \mathrm{H}), 7.84(\mathrm{~d}, J=8.4 \mathrm{~Hz}, 1 \mathrm{H}), 7.61(\mathrm{dd}$, $J=8.4,1.4 \mathrm{~Hz}, 1 \mathrm{H}), 4.45(\mathrm{~d}, J=14.7 \mathrm{~Hz}, 1 \mathrm{H}), 4.20(\mathrm{~d}, J=14.8 \mathrm{~Hz}, 1 \mathrm{H}), 3.88(\mathrm{~s}, 2 \mathrm{H}), 3.45(\mathrm{dd}, J=11.4,4.2 \mathrm{~Hz}, 1 \mathrm{H}), 2.08-2.13(\mathrm{~m}, 1 \mathrm{H}), 2.04-2.09(\mathrm{~m}, 1 \mathrm{H}), 1.96-2.03(\mathrm{~m}, 1 \mathrm{H})$, $1.77-1.82(\mathrm{~m}, 1 \mathrm{H}), 1.69($ br d, $J=3.2 \mathrm{~Hz}, 1 \mathrm{H}), 1.60-1.65(\mathrm{~m}, 1 \mathrm{H}), 1.66(\mathrm{br} \mathrm{d}, J=4.1 \mathrm{~Hz}, 1 \mathrm{H}), 1.56(\mathrm{~d}, J=3.0 \mathrm{~Hz}, 6 \mathrm{H}), 1.28(\mathrm{~s}, 3 \mathrm{H})$ 


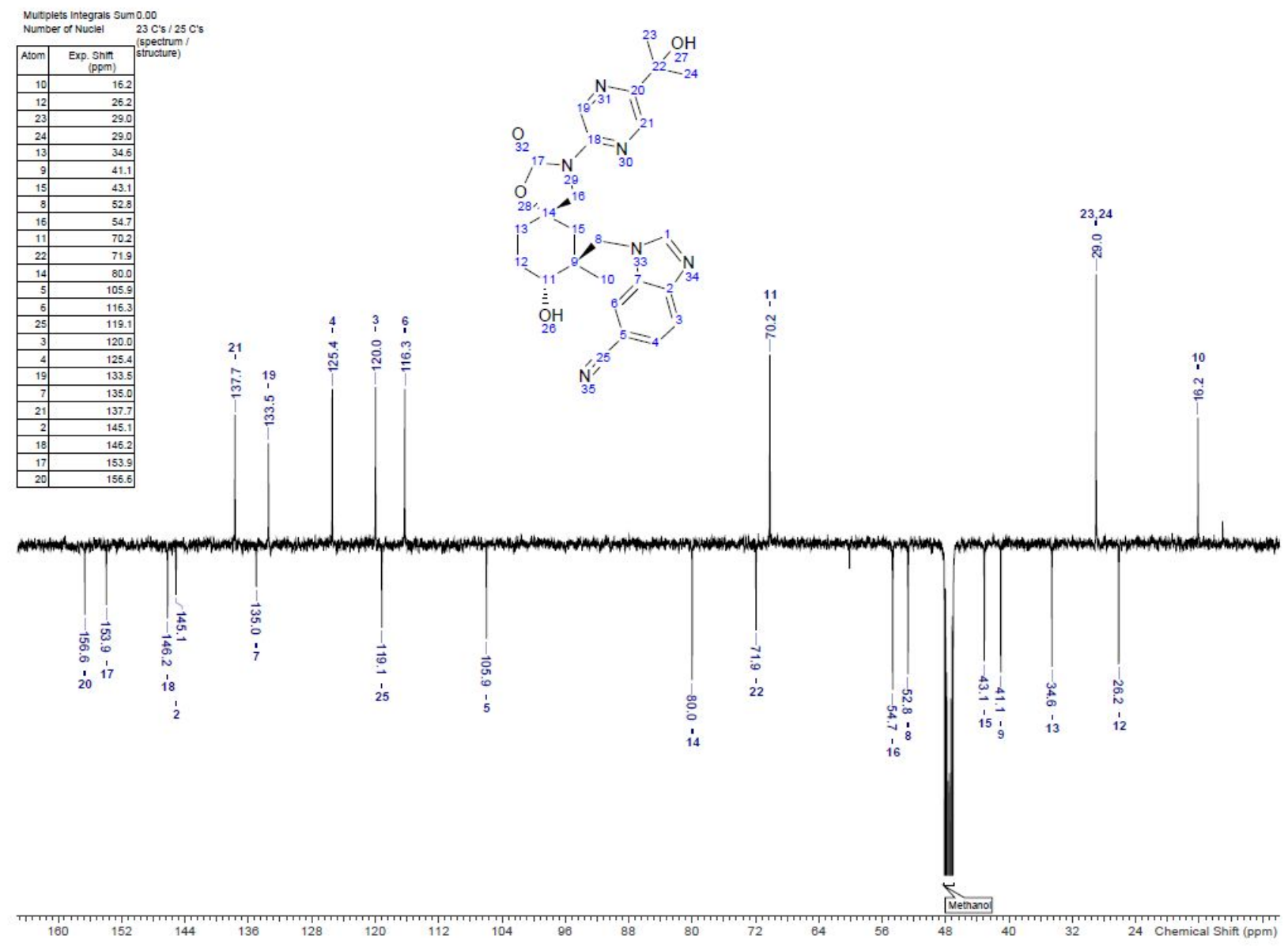

${ }^{13}{ }^{13}$ NMR (METHANOL-d $\left.4,126 \mathrm{MHz}\right): \bar{\delta}(\mathrm{ppm}) 156.6,153.9,146.2,145.1,137.7,135.0,133.5,125.4,120.0,119.1,116.3,105.9,80.0,71.9,70.2,54.7,52.8,43.1,41.1,34.6,29.0,26.2,16.2$ 


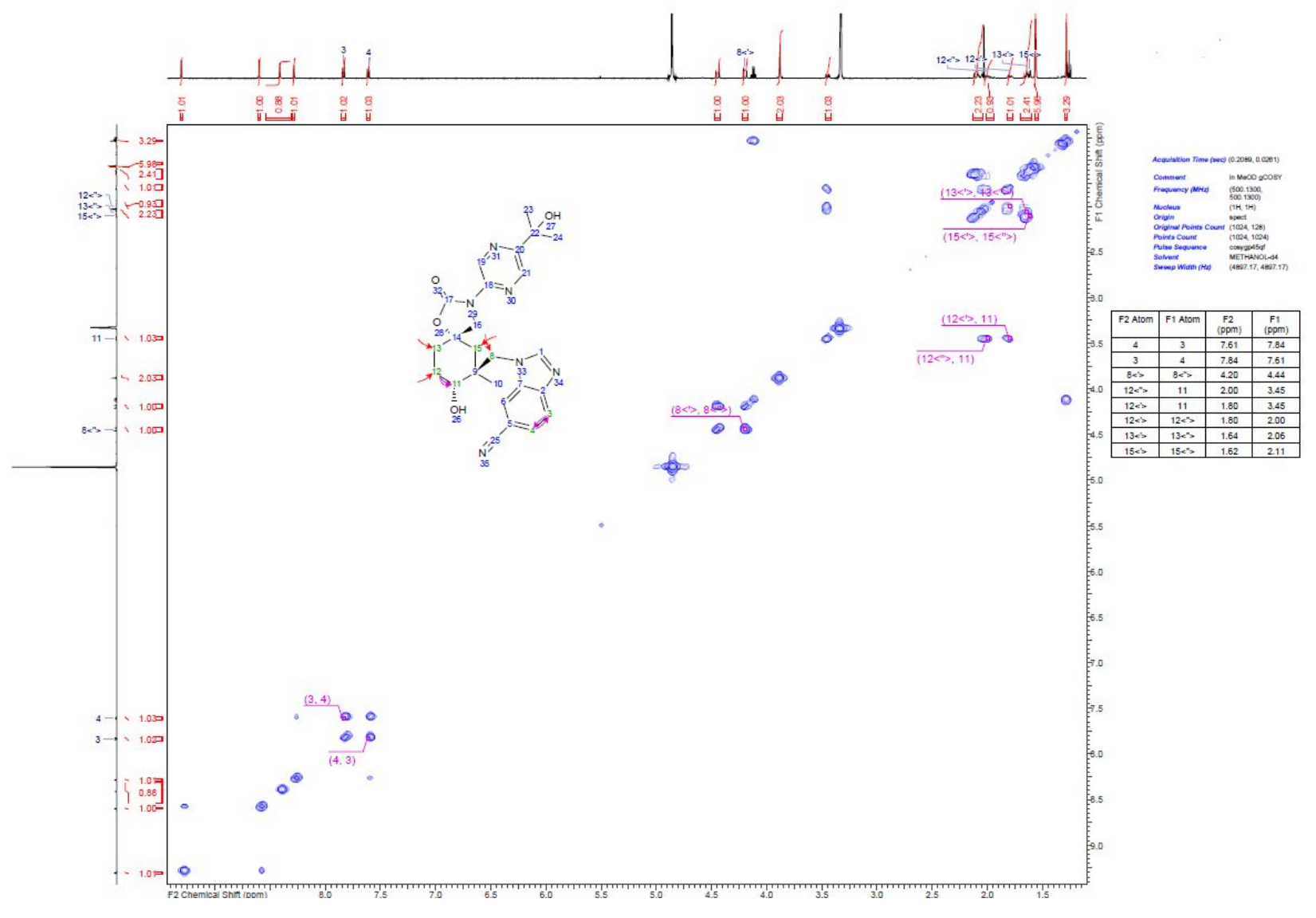




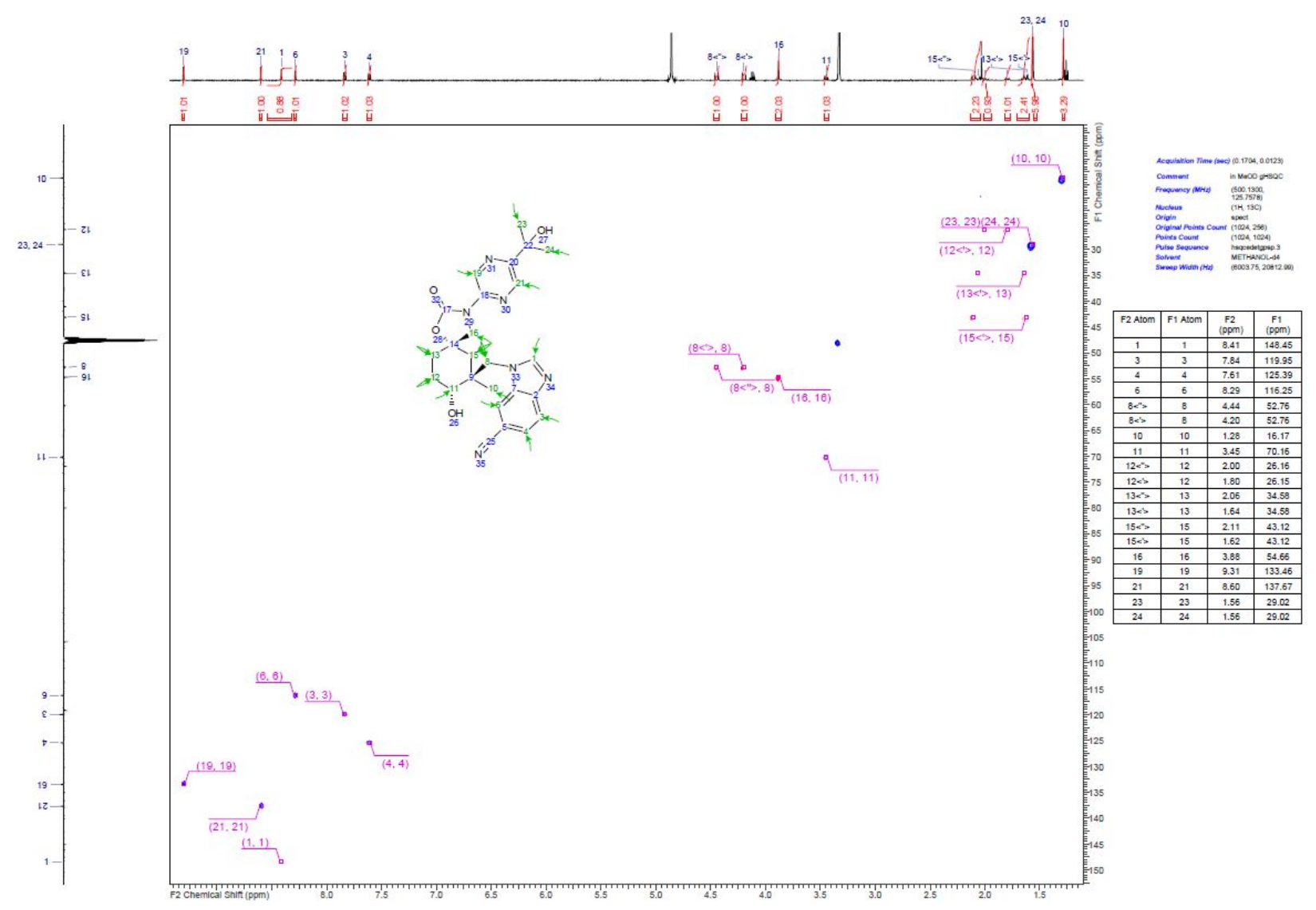




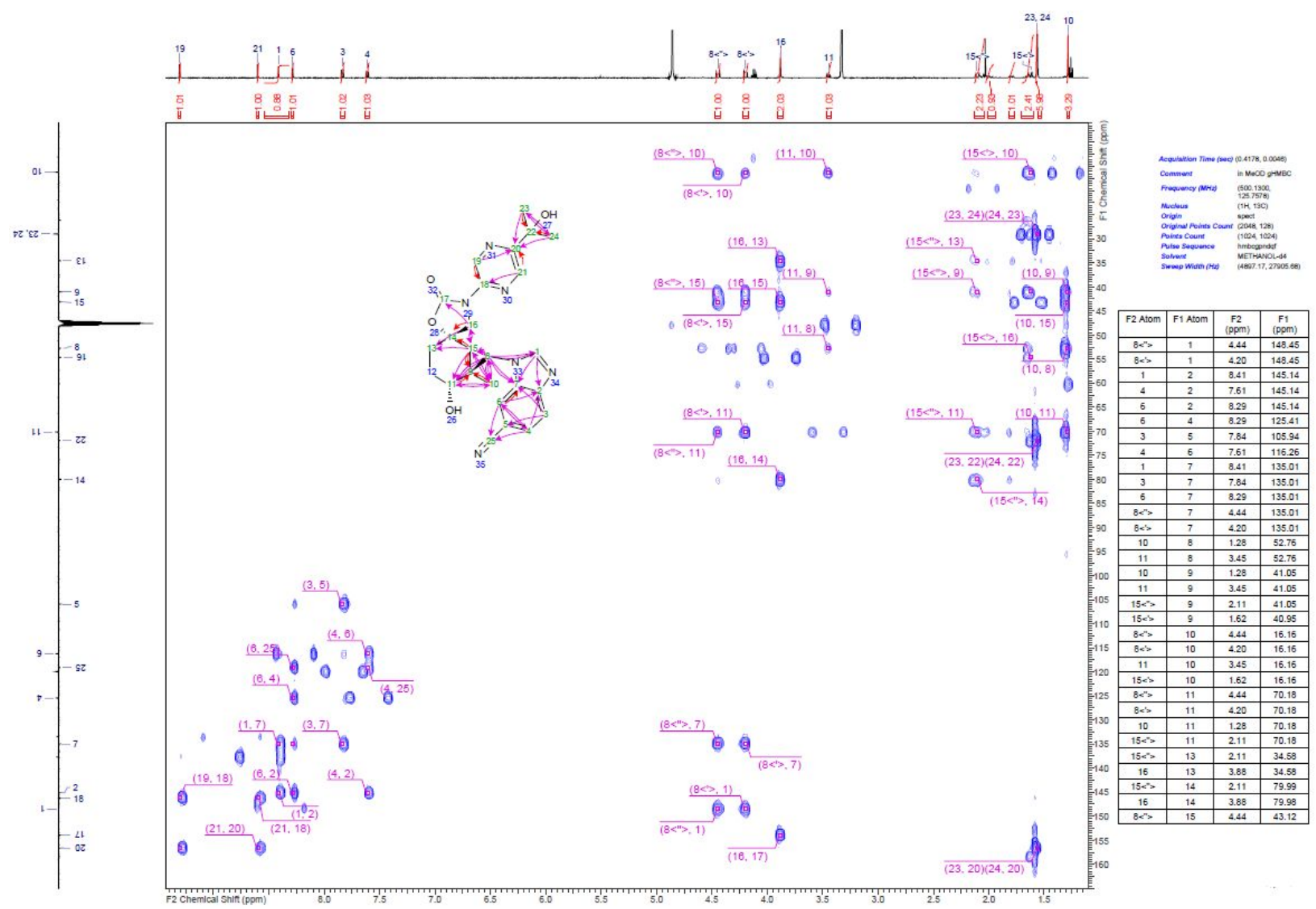

\begin{tabular}{|c|c|c|c|}
\hline F2 Atom & F1 Atom & $\begin{array}{c}\text { F2 } \\
\text { (ppm) }\end{array}$ & $\begin{array}{c}\text { F1 } \\
\text { (ppm) }\end{array}$ \\
\hline $8<>$ & 15 & 4.20 & 43.12 \\
\hline 10 & 15 & 1.28 & 43.12 \\
\hline 16 & 15 & 3.88 & 43.12 \\
\hline $15<^{\prime}>$ & 16 & 1.62 & 54.68 \\
\hline 16 & 17 & 3.88 & 153.93 \\
\hline 19 & 18 & 9.31 & 146.19 \\
\hline 21 & 18 & 8.60 & 146.20 \\
\hline 19 & 20 & 9.31 & 156.64 \\
\hline 21 & 20 & 8.60 & 156.65 \\
\hline 23 & 20 & 1.56 & 156.65 \\
\hline 24 & 20 & 1.56 & 156.65 \\
\hline 23 & 22 & 1.56 & 71.91 \\
\hline 24 & 22 & 1.56 & 71.91 \\
\hline 24 & 23 & 1.56 & 29.02 \\
\hline 23 & 24 & 1.56 & 29.02 \\
\hline 4 & 25 & 7.61 & 119.14 \\
\hline 6 & 25 & 8.29 & 119.14 \\
\hline
\end{tabular}



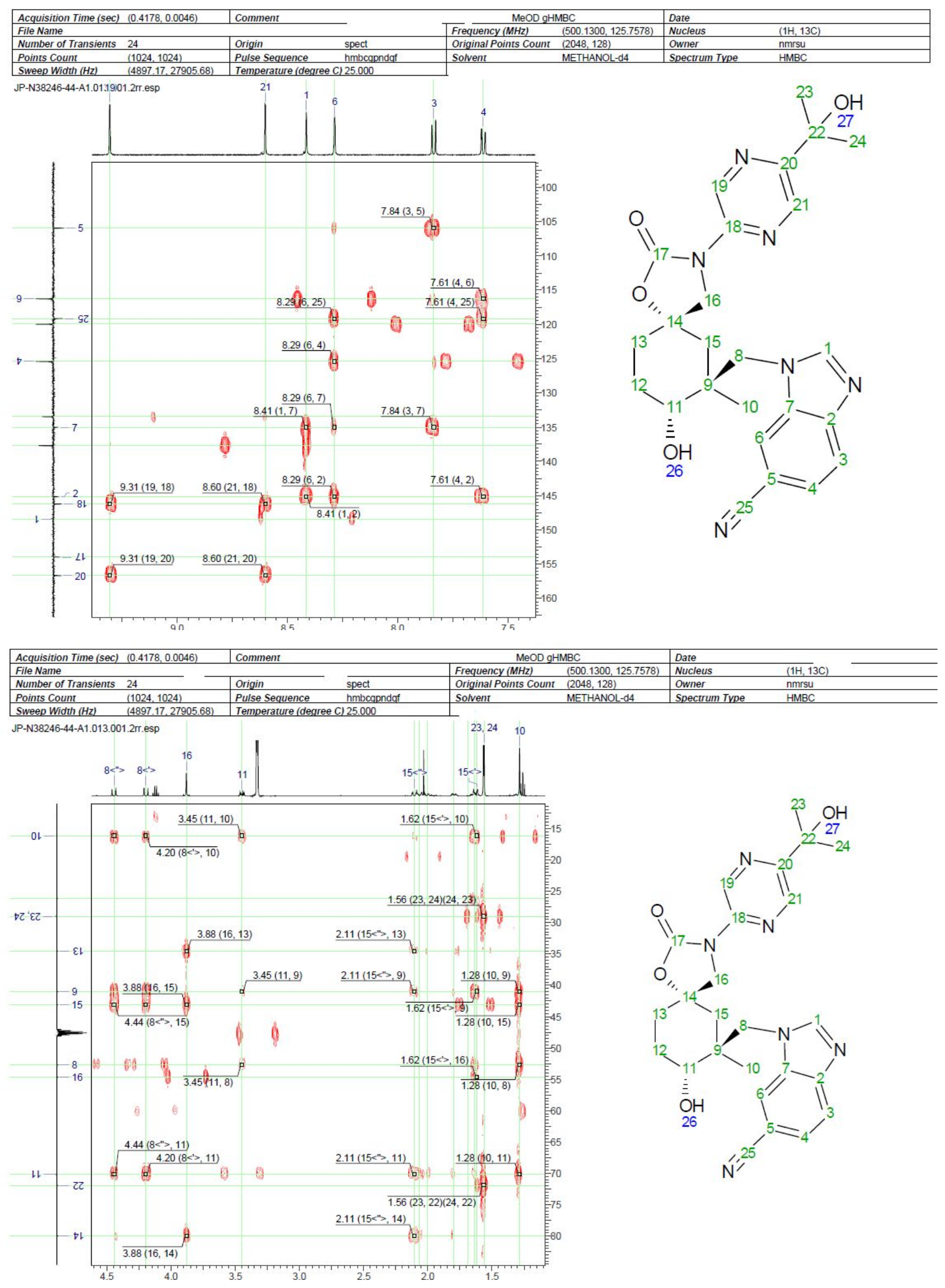


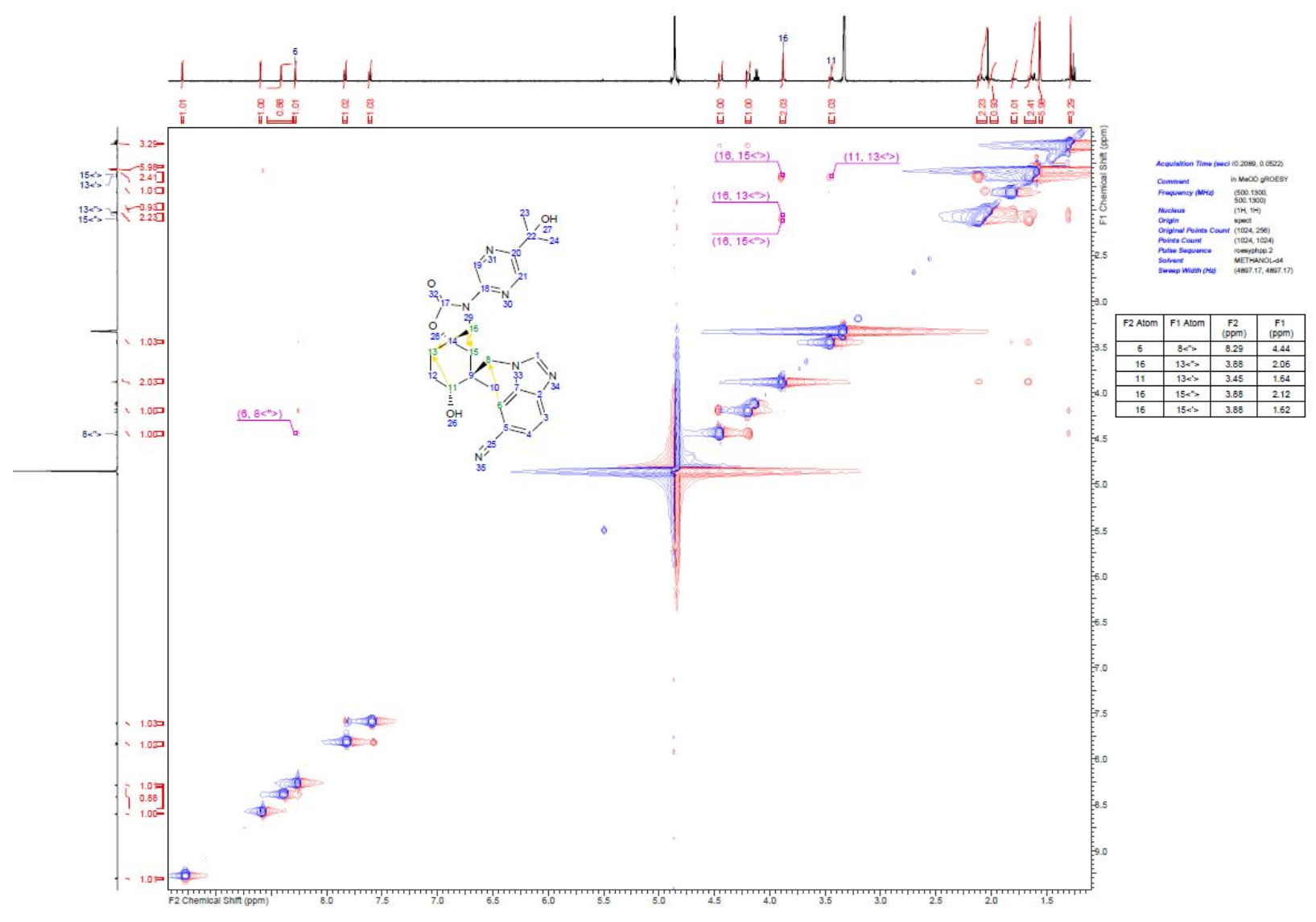

$A b$ initio VCD analysis of 1 and (ent)-1:
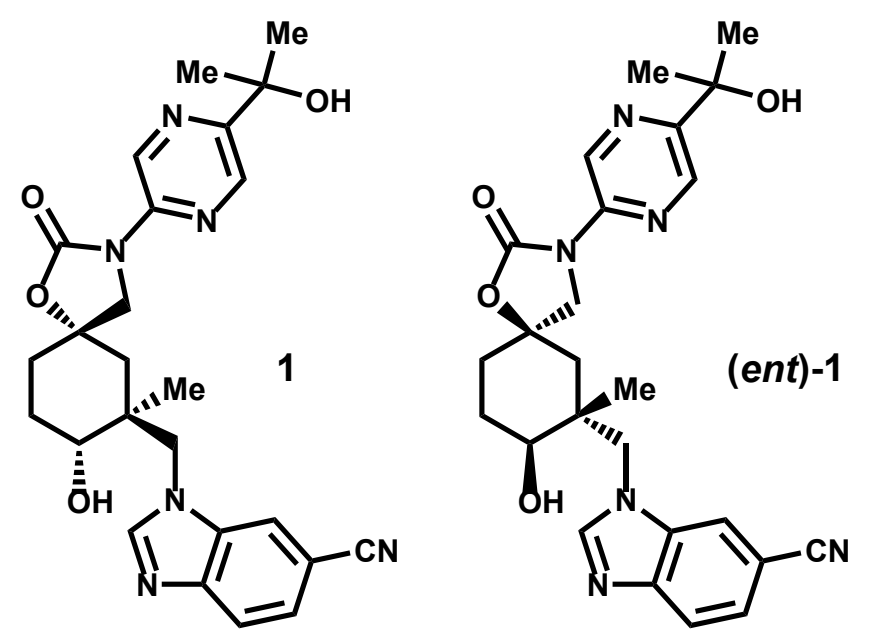

Theoretical analysis: 


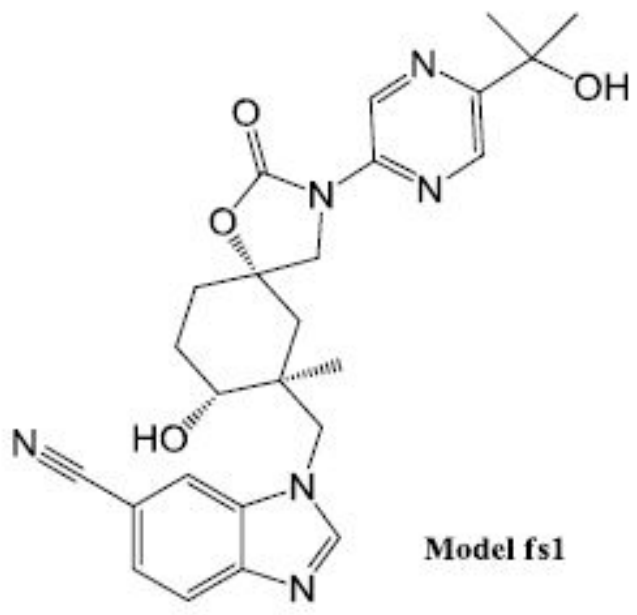

1-(( $(5 S, 7 S, 8 R)-8$-hydroxy-3-(5-(2-hydroxypropan-2-yl)pyrazin-2-yl)-7-methyl-2-oxo-1-oxa-3azaspiro[4.5]decan-7-yl)methyl)-1H-benzo[d] imidazolo-6-carbonitrile

- Conformational Search: MOE LowMode csearch using (a) Amber12:EHT ff; (b) rmsd=1.00; (c) Born solv (diel=ext=40)

- Model Chemistry: opt freq=(noraman,vcd) b3lyp/dgdzvp2 scrf=(solvent=ethanol)

- Conformational Analysis: Fractional populations estimated using best fit refinement from Boltzman populations.

- Lorentzian bandwidth: $8 \mathrm{~cm}-1$

- Frequency scale factor: 0.9825

- Estimation of Confidence Limit: CompareVOA (BioTools, Inc.) analysis

\section{Experimental:}

Spectrometer: Biotools ChirallR-2X FT-VCD spectrometer operating at $4 \mathrm{~cm}^{-1}$

- Frequency Range: $2200-800 \mathrm{~cm}^{-1}$

- PEM Calibration: dual PEM calibrated at $1400 \mathrm{~cm}^{-1}$

- $\quad$ PEM Retardation Settings: PEM1 $=0.250 \lambda$; PEM2 $=0.245 \lambda$

- ChirallR-2X Scan Methods:

- 81-E1: four $1 \mathrm{~h}$ blocks $(4 \times 3120$ total scans)

- 81-E2: four $1 \mathrm{~h}$ blocks (4 x 3120 total scans)

- Solvent: DMSO- $d_{6}$

- Concentrations:

- 1: $11.6 \mathrm{mg} / 215 \mu \mathrm{L}$

- (ent)-1: $12.1 \mathrm{mg} / 225 \mu \mathrm{L}$

- Baseline Correction (modified half-difference method):

- $\operatorname{VCDE} 1$ (corr'd) = VCDE1 - VCDE2

- $\operatorname{VCDE2}($ corr'd) $=$ VCDE2 - VCDE1

- Additional Processing: Savitsky-Golay 9-point smooth

Results: Analysis of Experimental and Calculated Data: 
The baseline-corrected VCD spectra for 1 and (ent)-1 are compared in Figure 1 with the VCD spectrum calculated for Model fs1. The green box in the panel highlights the spectral range over which VCD data were used to assign stereochemistry and estimate the confidence limit. Inspection of VCD data in the analysis range indicates that the VCD spectrum for Model fs1 is a close match with experimental of 1 . These findings are consistent with 1 having the same absolute configuration as Model fs 1 and (ent)-1 the opposite absolute configuration of Model fs 1 . Based on these results, 1 was assigned with $(5 S, 7 S, 8 R)$-absolute configuration, and (ent)-1 with $(5 R, 7 R, 8 S)$-absolute configuration. The confidence limit for this assignment was estimated to be $>99 \%$, consistent with the highest level of reliability.

The IR spectra for 1 and (ent)-1 are compared in Figure 2 with the IR spectrum calculated for Model fs1. As reported, the model spectrum is in very good qualitative agreement with experimental, confirming the overall structure of this sample (i.e., its molecular connectivity) and providing additional support for satisfactory coverage of its solution phase conformational space by the computational analysis.
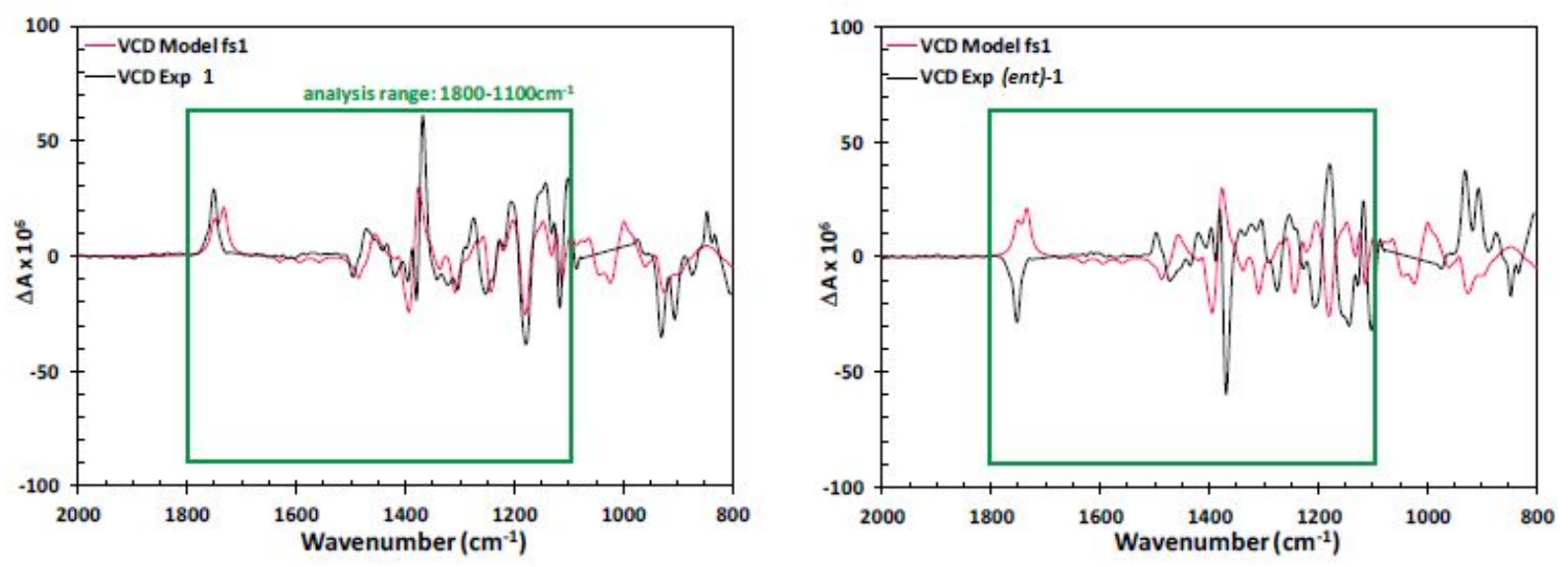

Figure 1: VCD comparisons for 1 and (ent)-1 vs Model fs 1. 

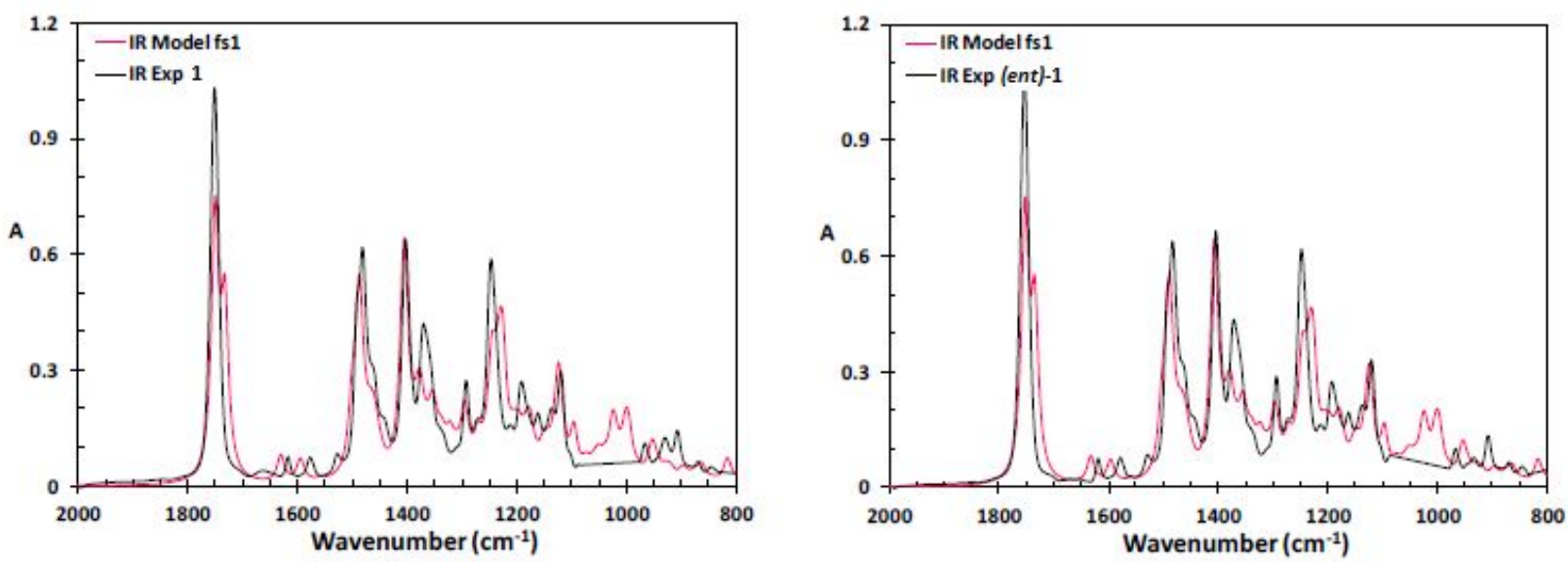

Figure 2: IR comparisons for 1 (left) and (ent)-1 (right) vs Model fs1.

\section{Estimated Level of Reliability}

- The confidence limit in this study was estimated using CompareVOATM (BioTools, Inc.), an automated tool for quantifying the level of agreement between calculated and observed VCD data.

- The confidence limit was determined from the absolute values of two parameters from CompareVOATM: total neighborhood similarity (TNS (VCD)) and the enantiomeric similarity index (ESI).

- The confidence limit was then determined by comparing these values to the ranges reported in the table:

\begin{tabular}{|c|c|c|c|}
\hline Reliability & $\begin{array}{c}{ }^{*} \text { TNS (VCD) } \\
\text { (range) }\end{array}$ & $\begin{array}{c}{ }^{*} \text { ESI } \\
\text { (range) }\end{array}$ & $\begin{array}{c}\text { Confidence Limit (CL) } \\
\text { (range) }\end{array}$ \\
\hline High & $\geq 70$ & $\geq 60$ & $>99 \%$ \\
\hline Medium & $60-70$ & $50-60$ & $95-99 \%$ \\
\hline Low & $50-60$ & $40-50$ & $90-95 \%$ \\
\hline Unreliable & $<50$ & $<40$ & $<90 \%$ \\
\hline
\end{tabular}

*absolute value

CompareVOA results for the current study:

- Analysis range: $1800-1100 \mathrm{~cm}^{-1}$

- Region omitted: none

- Range of statistical analysis (minimum $400 \mathrm{~cm}-1$ ): $700 \mathrm{~cm}^{-1}$

- Width of triangular weighting function: $15 \mathrm{~cm}^{-1}$

- TNS (VCD): 73.5 (absolute value)

- ESI: 69.4 (absolute value)

- Estimated Confidence Limit: > 99\%

- CompareVOA Graphical Result: 


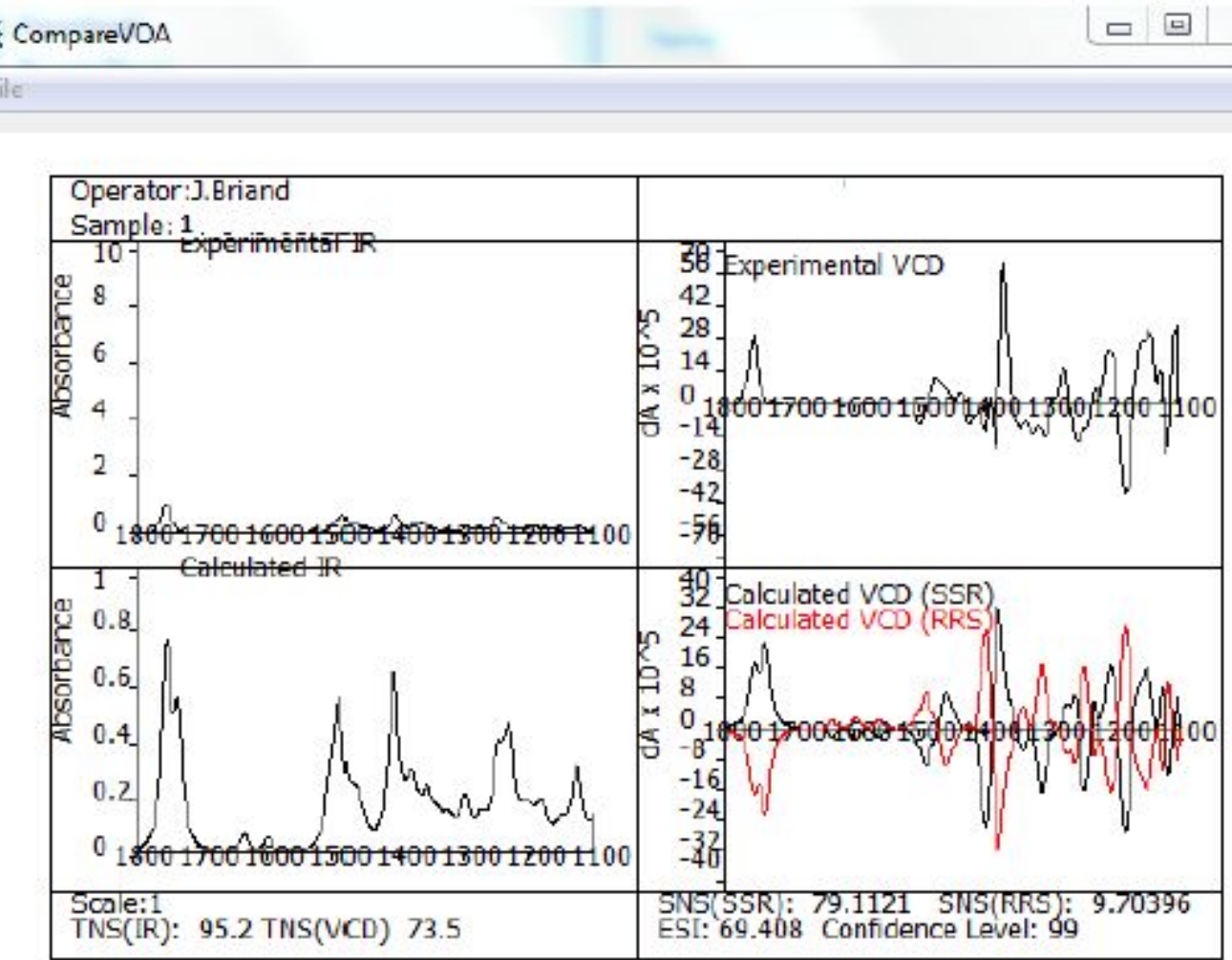

- ESI Statistical Plot: [Note: The red $x$ lies on the std. ref. line, indicating that CompareVOA statistics are consistent with 1 having the same absolute configuration as the model.] 


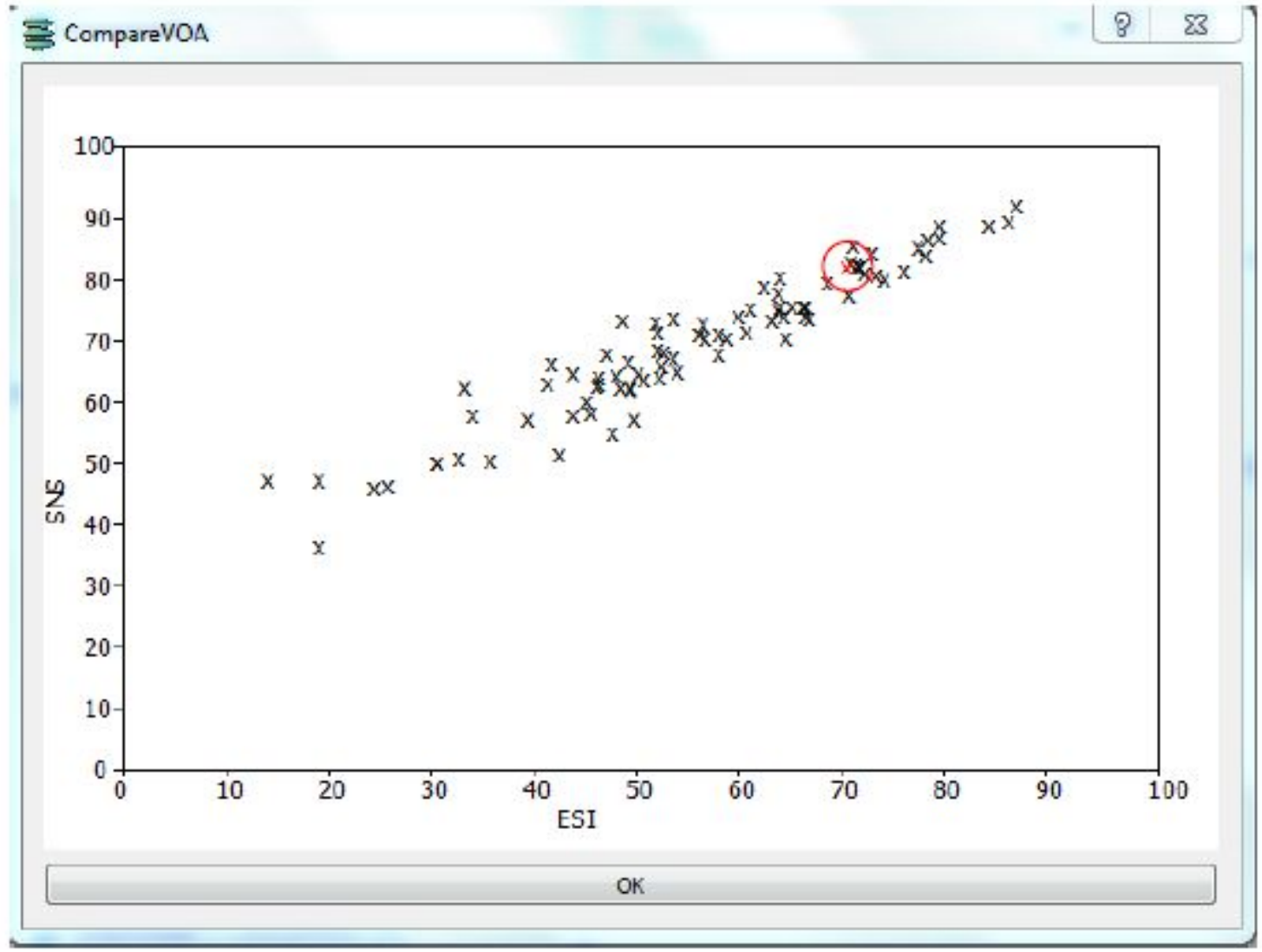


3. Comparisons of synthetic 1 to authentic GSK2708745 M1 GSK2798745 M1 NMR spectra:

$1 \mathrm{H}$

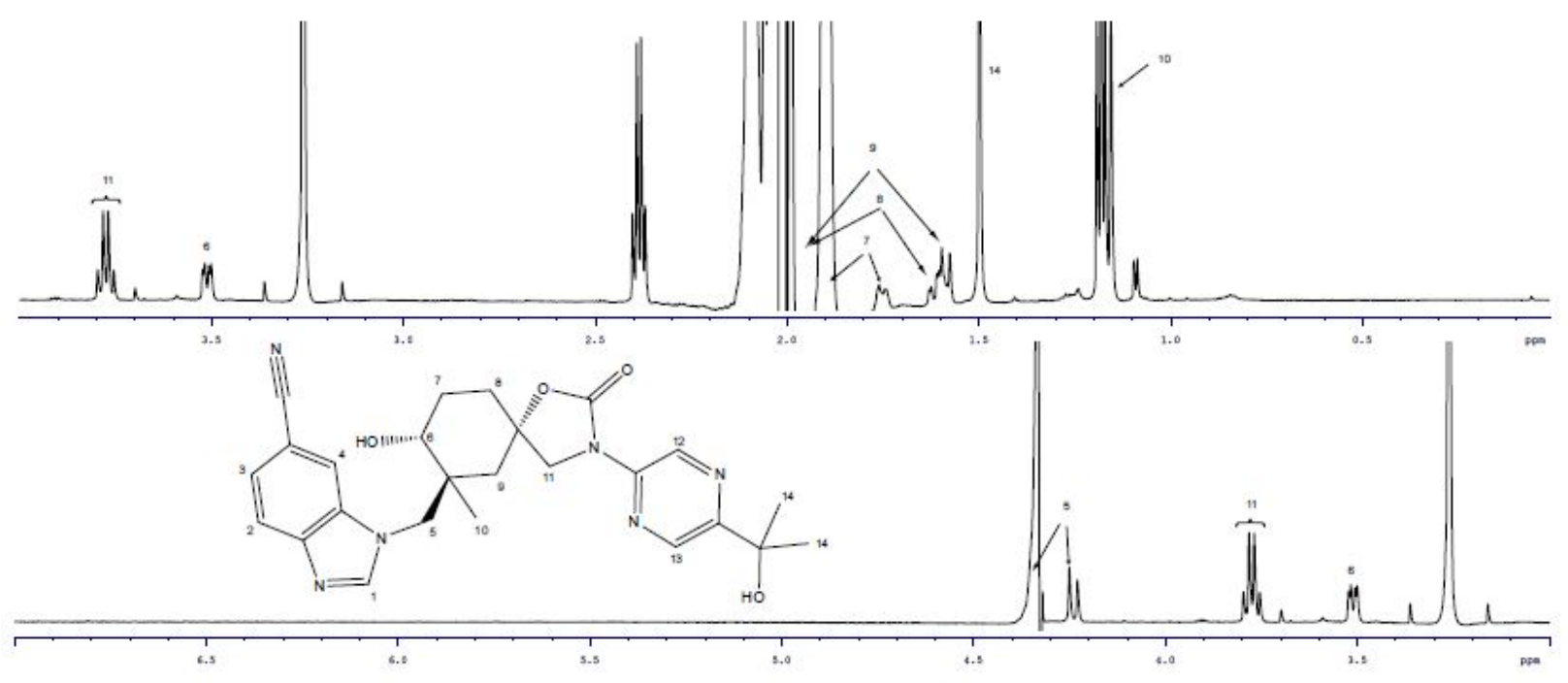

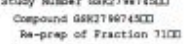

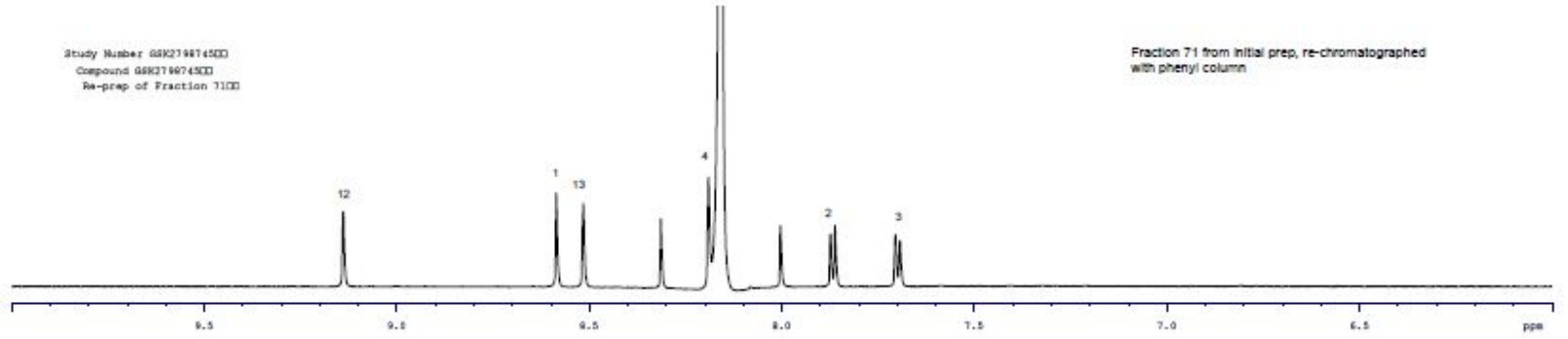

Fraction 71 trom initas prep, re-cromatopraphed
wath phenyl column 


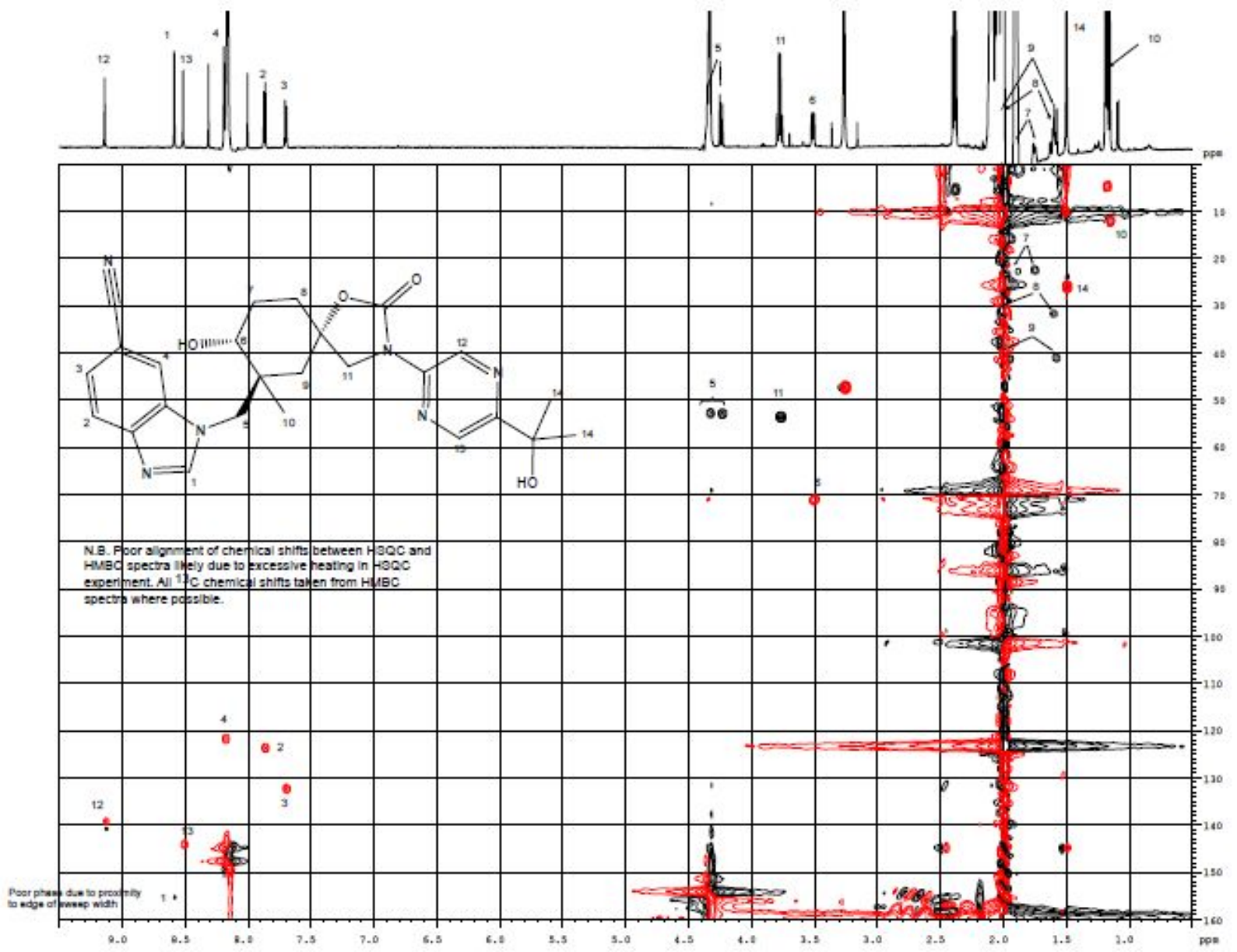


HMBC

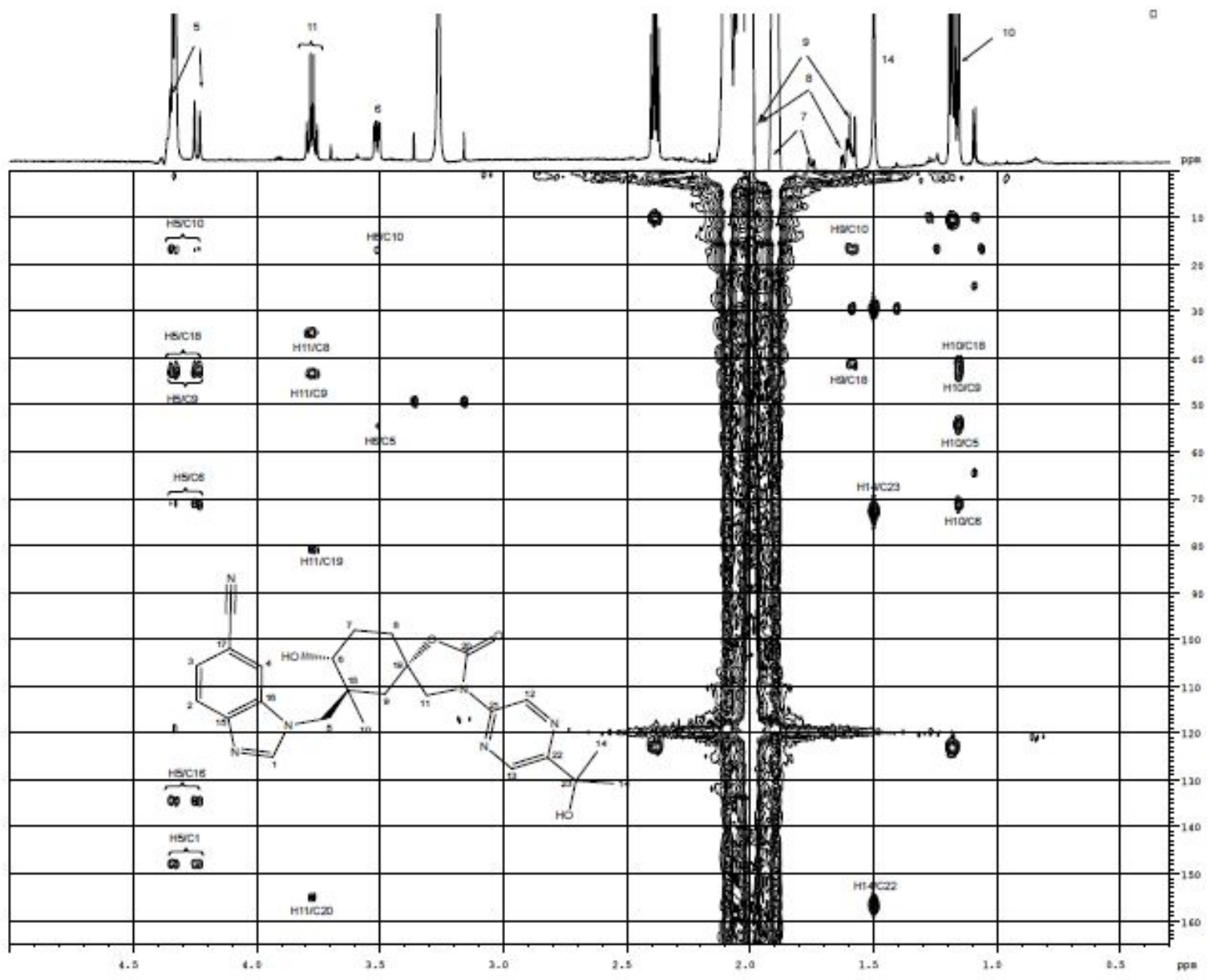


HMBC

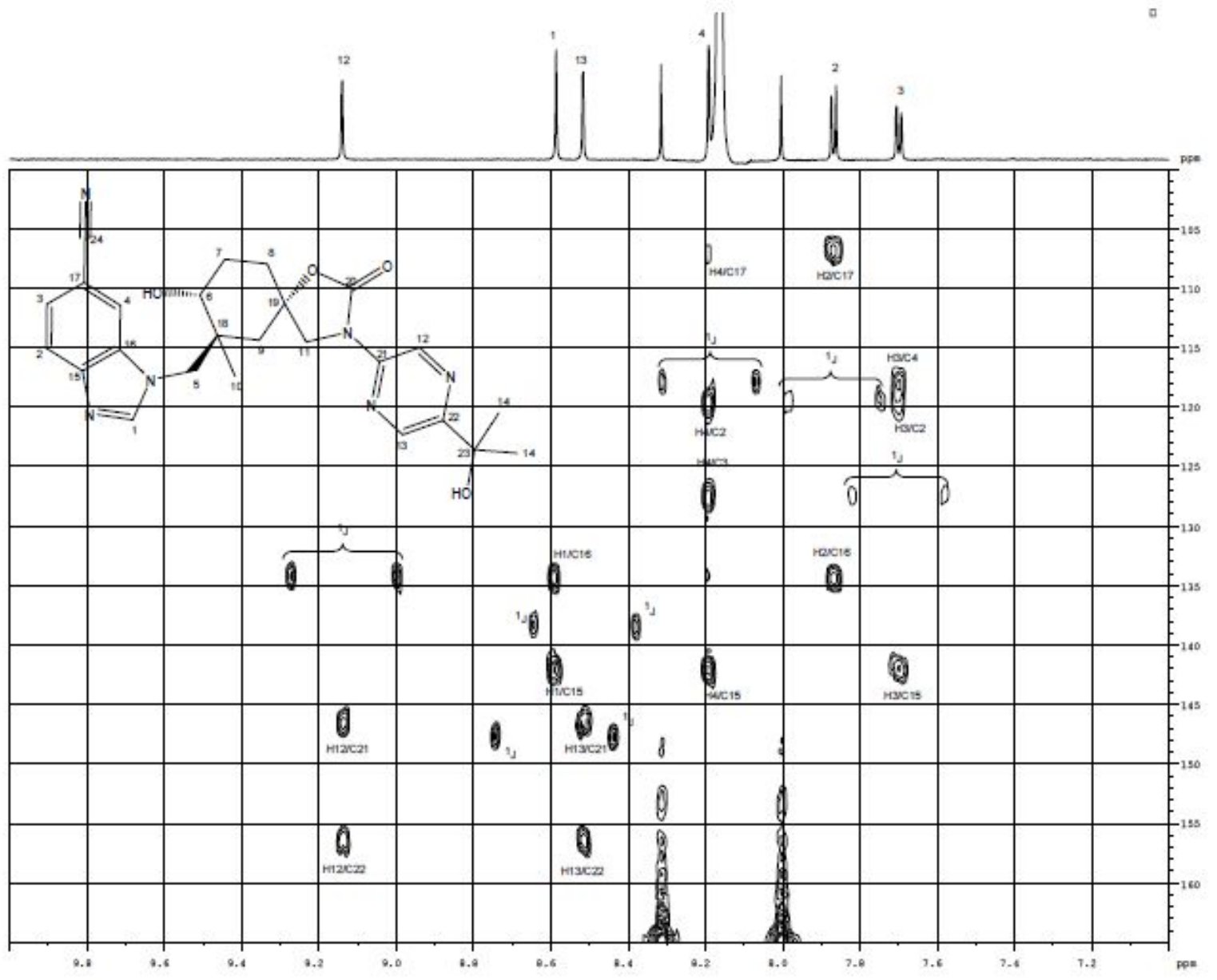




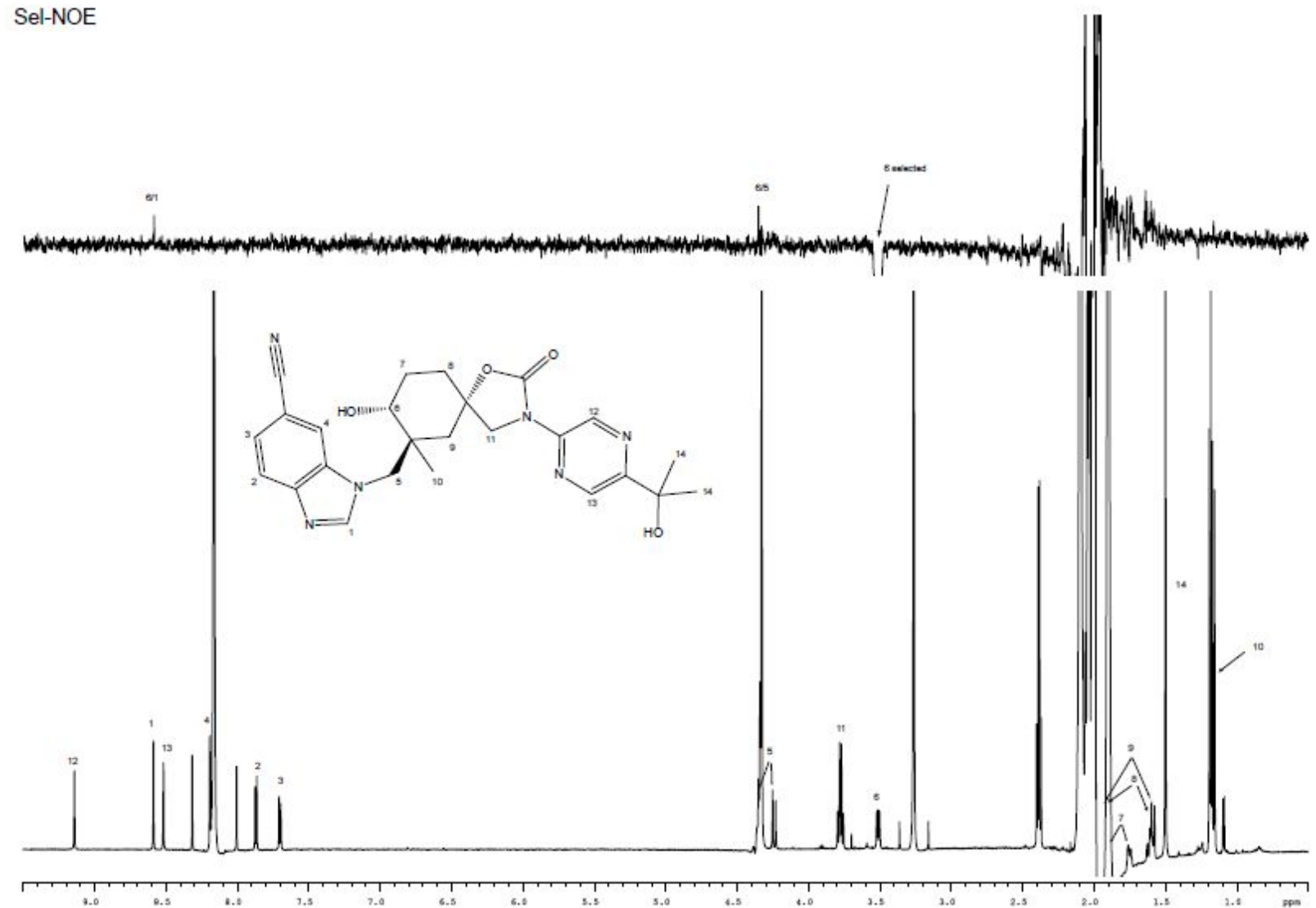




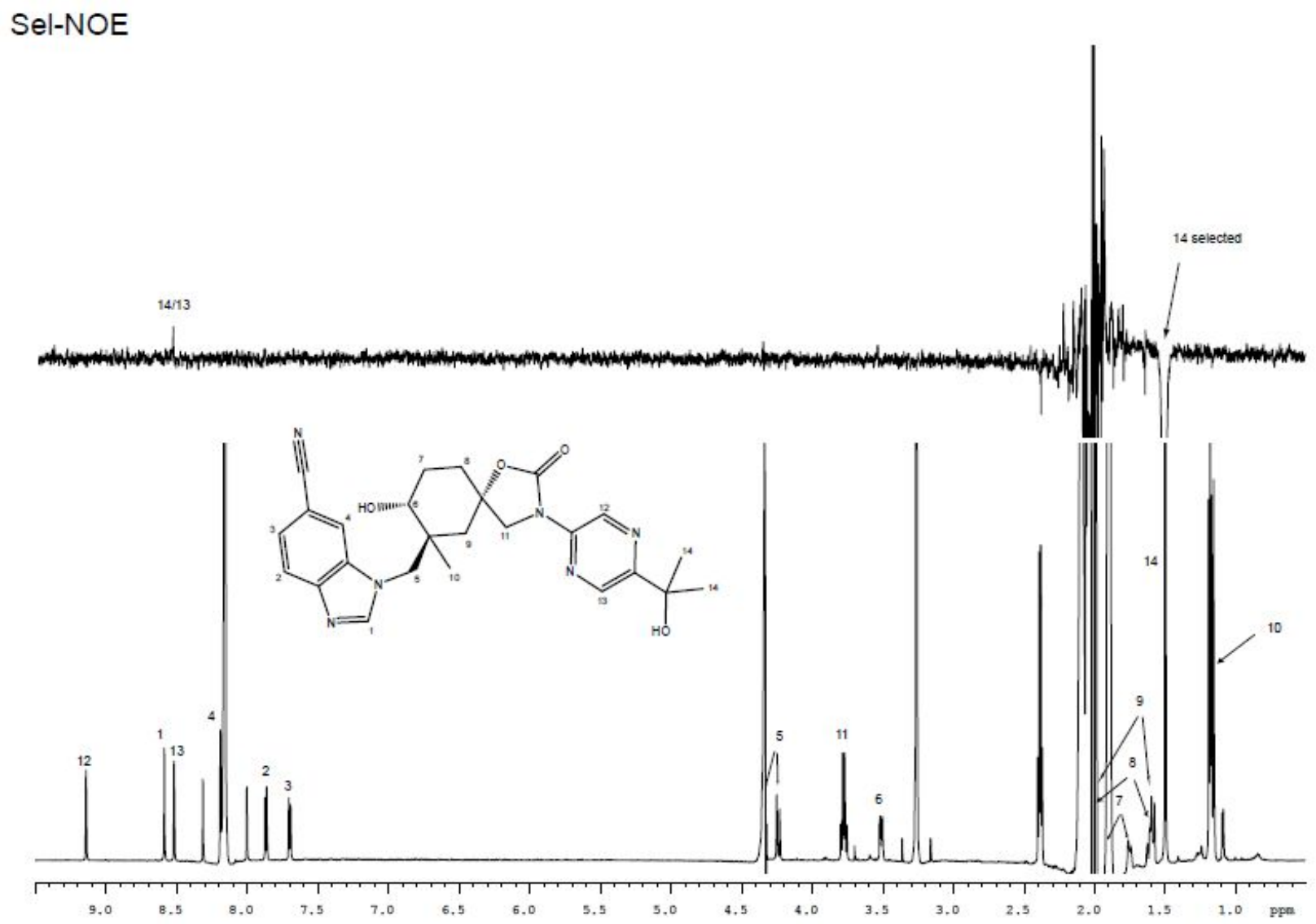


Co-NMR (50:50) of 1 and authentic metabolite (GSK2798745 M1) isolated from human samples:

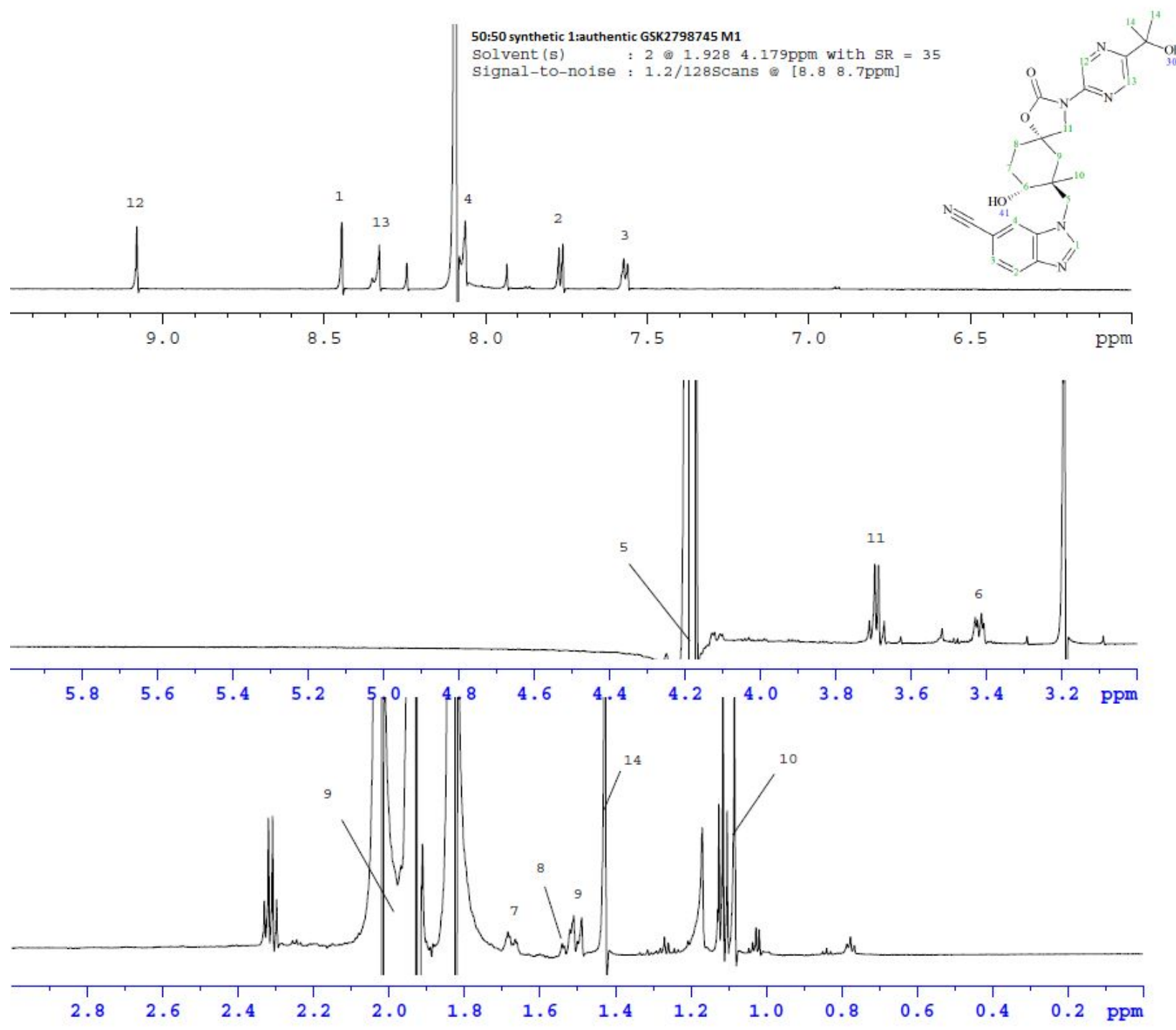




\section{Analytical HPLC comparisons:}

OH METABOLITE sample (1:5 diln)

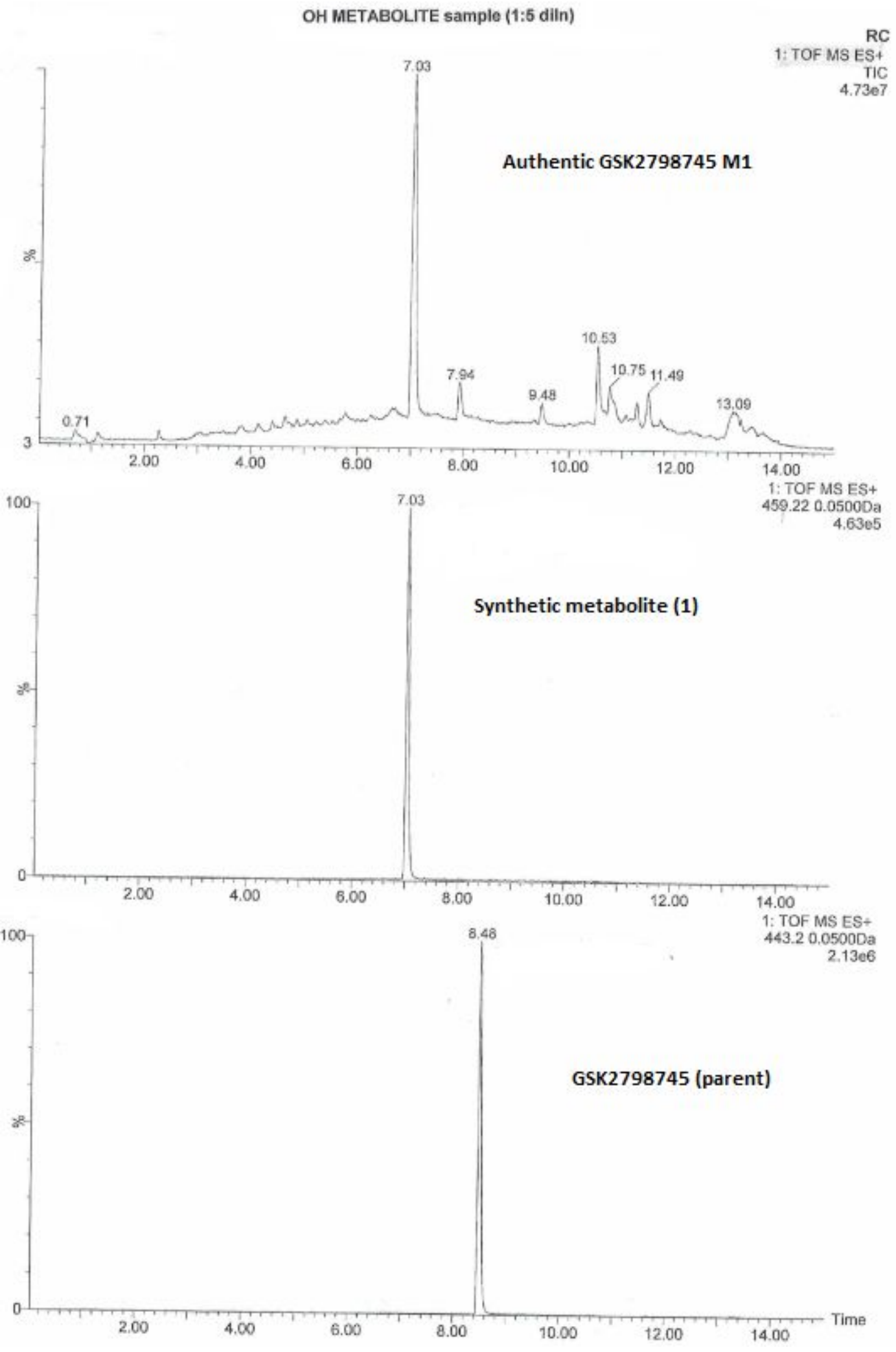



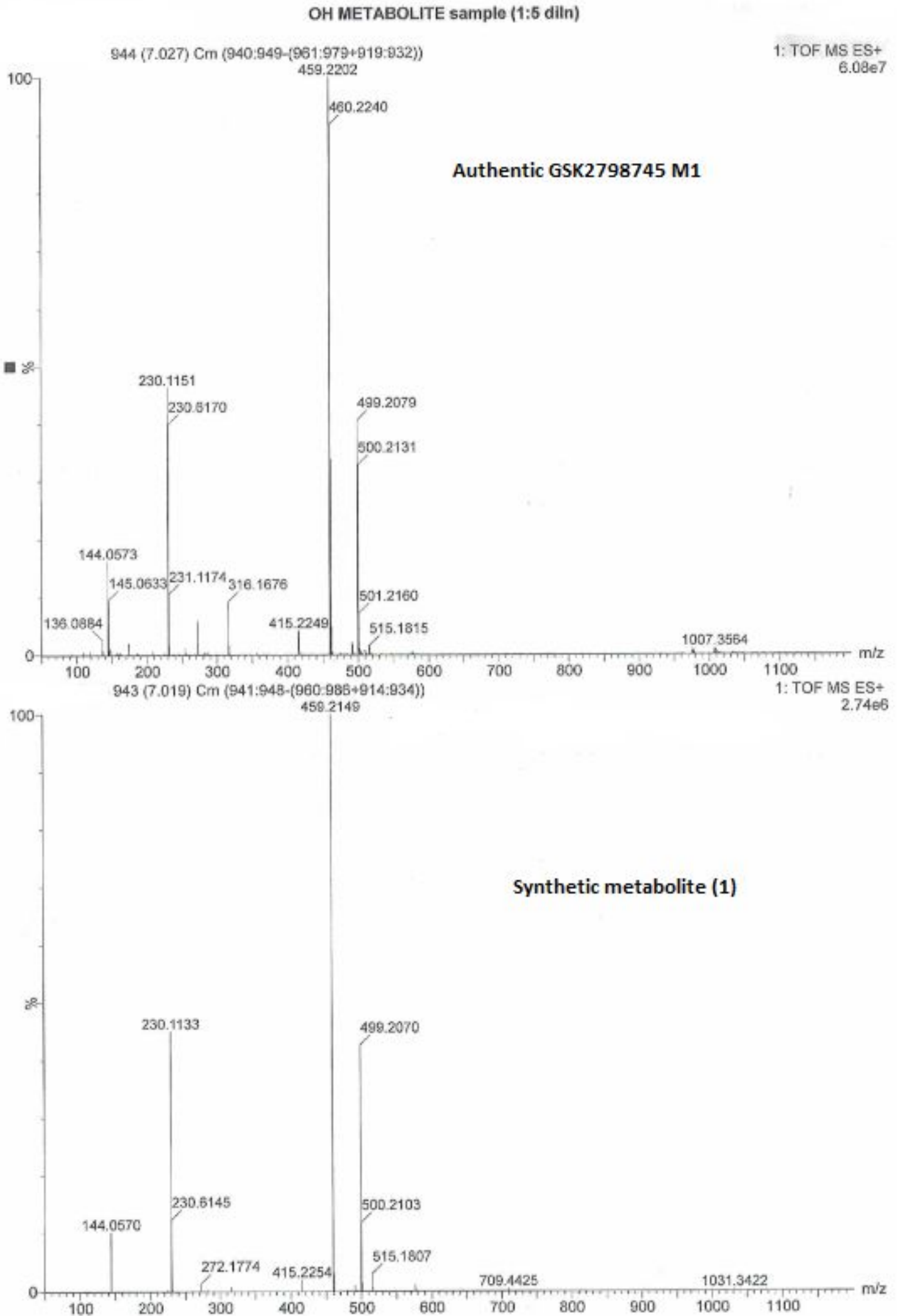
OH METABOLITE sample (1:6 diln)

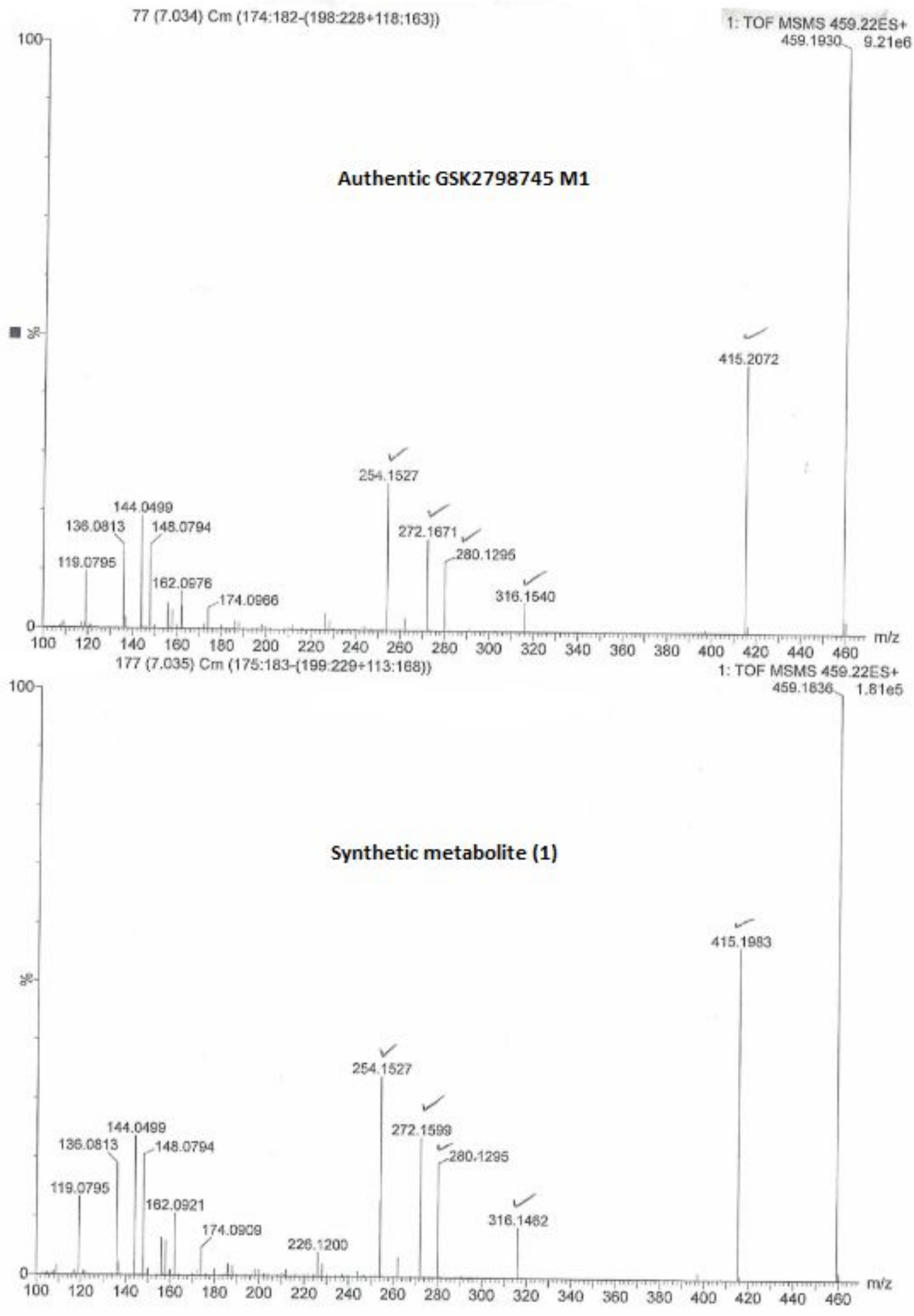

